\title{
MATRIX FACTORIZATIONS IN HIGHER CODIMENSION
}

\author{
JESSE BURKE AND MARK E. WALKER
}

\begin{abstract}
We observe that there is an equivalence between the singularity category of an affine complete intersection and the homotopy category of matrix factorizations over a related scheme. This relies in part on a theorem of Orlov. Using this equivalence, we give a geometric construction of the ring of cohomology operators, and a generalization of the theory of support varieties, which we call stable support sets. We settle a question of Avramov about which stable support sets can arise for a given complete intersection ring. We also use the equivalence to construct a projective resolution of a module over a complete intersection ring from a matrix factorization, generalizing the wellknown result in the hypersurface case.
\end{abstract}

\section{Contents}

1. Introduction

2. Matrix factorizations of locally free sheaves and Orlov's Theorem

3. Eisenbud operators

4. Proof of Theorem 3.2

5. Projective resolutions

6. A description of $\Psi^{-1}$ on objects

7. Properties of matrix factorizations

8. Stable support

Appendix A. Orlov's Theorem

Appendix B. Stable Ext-modules and complete resolutions

\section{INTRODUCTION}

Given an element $f$ in a commutative local ring $Q$, a matrix factorization of $f$ is a pair of $n \times n$ matrices $(A, B)$ such that $A B=f \cdot I_{n}=B A$. This construction was introduced by Eisenbud to study modules over the factor ring $R=Q /(f)$. Indeed, in the case $Q$ is regular local and $f \neq 0$, he showed [15, Theorem 6.1] that for any finitely generated $R$-module $M$, the minimal free resolution of the $d$-th syzygy of $M$, where $d$ is the Krull dimension of $Q$, is of the form

$$
\cdots \rightarrow R^{n} \stackrel{B \otimes_{Q} R}{\longrightarrow} R^{n} \stackrel{A \otimes_{Q} R}{\longrightarrow} R^{n} \stackrel{B \otimes_{Q} R}{\longrightarrow} R^{n} \rightarrow 0
$$

for some matrix factorization $(A, B)$. Thus, matrix factorizations describe the category of finitely generated $R$-modules, "up to finite projective dimension". This

Received by the editors November 20, 2012 and, in revised form, May 2, 2013.

2010 Mathematics Subject Classification. Primary 13D02, 14F05, 13 D09. 
was further clarified by Buchweitz in [11] where he noted that there is an equivalence of categories between the homotopy category of matrix factorizations and the quotient of the bounded derived category of finitely generated $R$-modules by perfect complexes:

$$
[M F(Q, f)] \stackrel{\cong}{\rightarrow} \mathrm{D}^{\mathrm{b}}(R) / \operatorname{Perf}(R)=: \mathrm{D}_{\mathrm{sg}}(R) .
$$

This functor sends a matrix factorization $(A, B)$ to coker $A$, regarded as an object of $\mathrm{D}_{\mathrm{sg}}(R)$. Here, the homotopy category of matrix factorizations is defined analogously to the homotopy category of complexes of modules. Also, a complex is perfect if it is isomorphic in $\mathrm{D}^{\mathrm{b}}(R)$ to a bounded complex of finitely generated projective $R$-modules. We call $\mathrm{D}_{\mathrm{sg}}(R)$ the singularity category of $R$, following [25].

In the equivalence (1.1), the right-hand side is well defined for any ring $R$.

Question 1. What should the left-hand side of (1.1) be when $R$ is a complete intersection - i.e., when $R=Q /\left(f_{1}, \ldots, f_{c}\right)$ for a regular ring $Q$ and a $Q$-regular sequence $f_{1}, \ldots, f_{c}$ ?

For a complete intersection $R=Q /\left(f_{1}, \ldots, f_{c}\right)$ we refer to $c$ as the codimension of $R$ (technically $c$ is the codimension of the presentation $Q /\left(f_{1}, \ldots, f_{c}\right)$ - but we fix a presentation throughout); complete intersections of codimension 1 are called hypersurfaces. In this paper we propose an answer for higher codimension complete intersections and investigate the consequences. To state it we introduce some notation. If $X$ is a scheme, $\mathcal{L}$ a line bundle on $X$, and $W$ a regular global section of $\mathcal{L}$, then a matrix factorization of the triple $(X, \mathcal{L}, W)$ consists of a pair of locally free coherent sheaves $\mathcal{E}_{1}, \mathcal{E}_{0}$ on $X$ and maps

$$
\mathcal{E}_{1} \stackrel{e_{1}}{\longrightarrow} \mathcal{E}_{0} \stackrel{e_{0}}{\longrightarrow} \mathcal{E}_{1} \otimes \mathcal{L}
$$

such that $e_{0} \circ e_{1}$ and $\left(e_{1} \otimes 1_{\mathcal{L}}\right) \circ e_{0}$ are both multiplication by $W$. The homotopy category of matrix factorizations, written $[M F(X, \mathcal{L}, W)]$, has these matrix factorizations as objects. To define the morphisms in this category one starts out as in the affine case, but some adjustment is needed to deal with the higher cohomology of the locally free sheaves involved. In Section 2 we recall the full definition of morphisms and give details on the following:

Theorem 1. Let $Q$ be a regular ring of finite Krull dimension, $f_{1}, \ldots, f_{c}$ a $Q$ regular sequence, and $R=Q /\left(f_{1}, \ldots, f_{c}\right)$. Let $X=\mathbb{P}_{Q}^{c-1}=\operatorname{Proj}\left(Q\left[T_{1}, \ldots, T_{c}\right]\right)$, $W=\sum_{i} f_{i} T_{i} \in \Gamma\left(X, \mathcal{O}_{X}(1)\right)$, and define

$$
Y=\operatorname{Proj}\left(Q\left[T_{1}, \ldots, T_{c}\right] /(W)\right) \hookrightarrow X
$$

to be the zero subscheme of $W$. There are equivalences of triangulated categories

$$
\left[M F\left(\mathbb{P}_{Q}^{c-1}, \mathcal{O}(1), W\right)\right] \underset{\text { coker }}{\stackrel{\cong}{\cong}} \mathrm{D}_{\mathrm{sg}}(Y) \underset{\Phi}{\stackrel{\cong}{\longrightarrow}} \mathrm{D}_{\mathrm{sg}}(R) .
$$

The first equivalence of this theorem has been proven in various contexts by various authors in [24, 28, 29]. The version we use here is from our previous paper [13. The second equivalence is essentially due to Orlov [26, Theorem 2.1]. In Appendix $\mathrm{A}$ we provide a slight generalization of loc. cit. removing the assumption that $Q$ is equicharacteristic and, more significantly, that $Q$ is regular. (When $Q$ is not regular, $D_{\mathrm{sg}}$ is replaced by the "relative singularity category".) This allows us to state many results in the paper in a "relative" form in which $Q$ is not necessarily 
regular but rather that the modules involved have finite projective dimension over $Q$.

The composition of the equivalences in Theorem 1, which we write

$$
\Psi=\Phi \circ \text { coker : }\left[M F\left(\mathbb{P}_{Q}^{\mathrm{c}-1}, \mathcal{O}(1), W\right)\right] \stackrel{\cong}{\longrightarrow} \mathrm{D}_{\mathrm{sg}}(R),
$$

provides an answer to Question 11. We note here that matrix factorizations of $W$ were (perhaps first) used in the proof of [4, 3.2] and their relation to MCM $R$-modules is currently being studied in work of Buchweitz, Pham, and Roberts; see [12. Also related is the work of Isik [20. In this paper we show that the above equivalence and non-affine matrix factorizations provide a new and useful perspective on many aspects of homological algebra over complete intersection rings. These results are naturally stated in terms of the stable Ext-modules of finitely generated $R$-modules $M, N$, defined as

$$
\widehat{\operatorname{Ext}}_{R}^{n}(M, N):=\operatorname{Hom}_{\mathrm{D}_{\mathrm{sg}}(R)}(M[-n], N), \quad n \in \mathbb{Z} .
$$

These modules are also known as the stable cohomology, or the Tate cohomology, of $M$ and $N$. When $R$ is Gorenstein it is a well-known result of Buchweitz that they may be computed using a complete resolution of $M$; see [11] and also Appendix B. The term "stable" reflects the facts that $\widehat{\operatorname{Ext}}_{R}^{n}(M, N)$ is zero if $M$ or $N$ has finite projective dimension and that there are natural isomorphisms $\operatorname{Ext}_{R}^{n}(M, N) \cong$ $\widehat{\operatorname{Ext}}_{R}^{n}(M, N)$ for $n \gg 0$.

When $R$ is a hypersurface it follows from (1.1) that there are natural isomorphisms

$$
\widehat{\operatorname{Ext}}_{R}^{n}(M, N) \cong \widehat{\operatorname{Ext}}_{R}^{n+2}(M, N)
$$

for all $n \in \mathbb{Z}$. For complete intersections of higher codimension the stable Extmodules are not necessarily two-periodic in this sense. They are, however, given as the sheaf cohomology modules of certain sheaves on $Y$ that do satisfy a "twisted" periodicity, as we now explain. For finitely generated $R$-modules $M$ and $N$, let $\mathcal{M}=\beta_{*} \pi^{*}(M)$ and $\mathcal{N}=\beta_{*} \pi^{*}(N)$, where $\pi: \mathbb{P}_{R}^{c-1} \rightarrow \operatorname{Spec}(R)$ is the canonical projection and $\beta: \mathbb{P}_{R}^{\mathrm{c}-1} \hookrightarrow Y$ is the evident closed immersion. (Here, we identify $M$ and $N$ with coherent sheaves on $\operatorname{Spec}(R)$ as usual.) We show in Section 3 (see Corollary 3.9 and Proposition 3.11) that for $n \gg 0$ there are natural isomorphisms

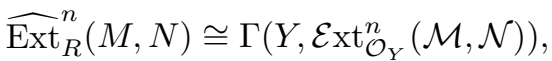

$$
\begin{aligned}
& \mathcal{E}_{\mathrm{xt}^{\prime}}^{n}(\mathcal{M}, \mathcal{N})(1) \cong \mathcal{E}_{\mathrm{Xt}_{\mathcal{O}_{Y}}^{n+2}}^{n+\mathcal{M}, \mathcal{N})} \text {, }
\end{aligned}
$$

where $\mathcal{E}_{\mathrm{xt}_{\mathcal{O}_{X}}}^{n}(\mathcal{M}, \mathcal{N})$ denotes the coherent sheaf satisfying

$$
\mathcal{E}_{\mathrm{xt}_{\mathcal{O}_{X}}}^{n}(\mathcal{M}, \mathcal{N})_{x} \cong \operatorname{Ext}_{\mathcal{O}_{X, x}}^{n}\left(\mathcal{M}_{x}, \mathcal{N}_{x}\right) .
$$

Combining these, there is an integer $m \geq 0$ and isomorphisms

$$
\begin{aligned}
& \widehat{\operatorname{Ext}}_{R}^{m+2 k}(M, N) \cong \Gamma\left(Y, \mathcal{E}_{\mathrm{xt}_{\mathcal{O}_{Y}}^{m}}^{m}(\mathcal{M}, \mathcal{N})(k)\right) \quad \text { for all } k \geq 0, \\
& \widehat{\operatorname{Ext}}_{R}^{m+2 k+1}(M, N) \cong \Gamma\left(Y, \mathcal{E}_{\left.\mathrm{xt}_{\mathcal{O}_{Y}}^{m+1}(\mathcal{M}, \mathcal{N})(k)\right)} \text { for all } k \geq 0 .\right.
\end{aligned}
$$

In other words, the even (respectively, odd) Ext-modules of $M$ and $N$ are given (in high enough degrees) by the graded components of the graded $Q\left[T_{1}, \ldots, T_{c}\right]$-module associated to the coherent $\mathcal{O}_{Y}$-sheaf $\mathcal{E}_{\mathrm{xt}^{m}}^{m}(\mathcal{M}, \mathcal{N})$ (respectively, $\mathcal{E}_{\mathrm{Xt}_{Y}}^{m+1}(\mathcal{M}, \mathcal{N})$ ). If $c=1$, then $Y=\operatorname{Spec} R$ and $\mathcal{L}$ is the trivial bundle, recovering the two-periodicity for hypersurfaces. 
The isomorphisms (1.3) show that in high degrees there are natural maps of degree two on the stable Ext-modules, given by multiplication by the variables $T_{1}, \ldots, T_{c}$. On the other hand, it is well known that

$$
\operatorname{Ext}_{R}^{*}(M, N)=\bigoplus_{n \geq 0} \operatorname{Ext}_{R}^{n}(M, N)
$$

is a graded module over the polynomial ring $R\left[\chi_{1}, \ldots, \chi_{c}\right]$ (which is graded by setting $\left|\chi_{i}\right|=2$ ), where the action of the $\chi_{i}$ 's is induced by the Eisenbud operators [15].

Theorem 3.2 shows that these two families of operators coincide under the isomorphisms $\widehat{\operatorname{Ext}}_{R}^{n}(M, N) \cong \operatorname{Ext}_{R}^{n}(M, N)$, for $n \gg 0$ :

Theorem 2. Let $M$ and $N$ be finitely generated $R$-modules. For $n \gg 0$ and $i=1, \ldots, c$ there is an equality:

$$
\chi_{i}=T_{i}: \widehat{\operatorname{Ext}}_{R}^{n}(M, N) \rightarrow \widehat{\operatorname{Ext}}_{R}^{n+2}(M, N) .
$$

The statement of this theorem and its consequences occupy Section 3 The (surprisingly delicate) proof makes up Section 4.

As outlined above, the equivalence (1.1) was first proved using the fact that the minimal free resolution of a module over a local hypersurface is eventually given by a matrix factorization. In Section 5 we go in the opposite direction and use the equivalence $\Psi$ to show:

Theorem 3. Let $M$ be a finitely generated $R$-module and $\mathbb{E}=\left(\mathcal{E}_{1} \rightarrow \mathcal{E}_{0} \rightarrow \mathcal{E}_{1}(1)\right)$ a matrix factorization of $\left(\mathbb{P}_{Q}^{c-1}, \mathcal{O}(1), W\right)$ such that

$$
\Psi(\mathbb{E}) \cong M \in \mathrm{D}_{\mathrm{sg}}(R) .
$$

There is an integer $n$, that depends on $\mathbb{E}$, such that the $n$-th syzygy of $M$ has a free resolution constructed from the sheaf cohomology of twists of $\mathcal{E}_{1}$ and $\mathcal{E}_{0}$.

See Theorem 5.2 for a precise description of this resolution. In Section 6 we show how to construct a matrix factorization $\mathbb{E}_{M}$ such that $\Psi\left(\mathbb{E}_{M}\right) \cong M$, using a finite resolution of $M$ over $Q$ and a system of higher homotopies, as in [15, §7]. Using this explicit construction we also show that one may describe $\mathrm{D}_{\mathrm{sg}}(R)$ using graded matrix factorizations of $W$ over the ring $Q\left[T_{1}, \ldots, T_{c}\right]$.

When $R$ is local, Avramov and Buchweitz in [4] use the action of $R\left[\chi_{1}, \ldots, \chi_{c}\right.$ ] on $\operatorname{Ext}_{R}^{*}(M, N)$ to define the notion of a support variety. Given finitely generated $R$-modules $M$ and $N$, their support variety is a closed subset of $\mathbb{A}_{k}^{c}$, which we write as

$$
V_{Q}^{\mathbf{f}}(M, N)^{A B} \subseteq \mathbb{A}_{k}^{c}=\operatorname{Spec} k\left[\chi_{1}, \ldots, \chi_{c}\right],
$$

where $k$ is the residue field of $R$.

For $R=Q /(\mathbf{f})$ a (not necessarily local) complete intersection, we define the stable support set of a pair of $R$-modules $M$ and $N$ to be

$$
\left.V_{Q}^{\mathbf{f}}(M, N):=\operatorname{supp} \operatorname{Ext}_{R}^{*(M}, N\right) \subseteq \mathbb{P}_{R}^{\mathrm{c}-1} .
$$

After establishing various formal properties of matrix factorizations in Section 7 which we will use, in Section 8 we show the properties of stable support sets listed in the theorem below. These generalize the properties of support varieties proven in 4]; see also [9, 5.1]. 
Theorem 4. For finitely generated R-modules $M, N, M^{\prime}$, and $N^{\prime}$, we have:

(1) $V_{Q}^{\mathbf{f}}(M, N)^{A B}$ is the cone of the closed fiber of $V_{Q}^{\mathbf{f}}(M, N)$ when $R$ is local;

(2) $V_{Q}^{\mathbf{f}}(M, N)=\emptyset$ if and only if $\operatorname{Ext}_{R}^{n}(M, N)=0$ for all $n \gg 0$;

(3) $V_{Q}^{\mathbf{f}}(M, N) \cap V_{Q}^{\mathbf{f}}\left(M^{\prime}, N^{\prime}\right)=V_{Q}^{\mathbf{f}}\left(M, N^{\prime}\right) \cap V_{Q}^{\mathbf{f}}\left(M^{\prime}, N\right)$; and

(4) $V_{Q}^{\mathbf{f}}(M, N)=V_{Q}^{\mathbf{f}}(M, M) \cap V_{Q}^{\mathbf{f}}(N, N)=V_{Q}^{\mathbf{f}}(N, M)$.

Moreover, it follows almost immediately from the definition that

$$
V_{Q}^{\mathbf{f}}(M, N)=V_{Q}^{\mathbf{f}}\left(\Omega_{R}^{n}(M), N\right)=V_{Q}^{\mathbf{f}}\left(M, \Omega_{R}^{n}(N)\right)
$$

for any $n \in \mathbb{Z}$, where $\Omega_{R}^{n}(M)$ is an $n$-th syzygy of $M$. (If $n<0$, then $\Omega_{R}^{n}(M)$ is defined if and only if there is a long exact sequence $0 \rightarrow M \rightarrow P_{0} \stackrel{\partial}{\rightarrow} \cdots \stackrel{\partial}{\rightarrow} P_{-n}$ with $P_{i}$ projective. In this case $\Omega_{R}^{n}(M)=P_{-n} / \partial\left(P_{-n+1}\right)$.)

One can check that stable support sets are contained in the singular locus of $Y$, which is a subset of $\mathbb{P}_{R}^{c-1}$; see Lemma 8.2. Thus the following result shows that every "conceivable" subset of $\mathbb{P}_{R}^{c-1}$ is the stable support set of an $R$-module. This answers in the affirmative a question suggested to us by Avramov, and it generalizes [10] and [5]; see also [32, 7.11].

Theorem 5. For every closed subset $C$ of the singular locus of

$$
Y=\operatorname{Proj}\left(Q\left[T_{1}, \ldots, T_{c}\right] /(W)\right),
$$

there exists an $R$-module $M$ with $C=V_{Q}^{\mathbf{f}}(M, M)$.

This theorem is proved in Section 8. Also in that section we show how notions of support for modules and complexes over complete intersections defined in [8,32] relate to stable support.

The paper also contains two appendices: Appendix $\mathrm{A}$ gives the generalization of Orlov's Theorem described above and Appendix B shows that if a module $M$ has a complete resolution in the sense of 34, then this resolution may be used to compute the stable Ext-modules as defined above. This is well known in the Gorenstein case (see [11]), but we could not find the result in the literature in the generality that we need here.

As alluded to above, we have endeavored to state and prove the results in this paper in a more general setting than discussed in this introduction. In particular, we do not typically assume $Q$ is a regular ring, but instead assume that $M$ (and occasionally $N$ ) has finite projective dimension over $Q$. This leads to somewhat more complicated statements than those listed in this introduction. To counteract this, we have also sought to include explicitly the "simple case" in which $Q$ is regular, so that the reader who is only interested in this case can find the results in a more pleasing and easy-to-understand format.

\section{MatriX FaCtorizations of LOCALLY FREE SHEAVES AND ORLOV'S THEOREM}

In this section, we summarize relevant results from our previous paper 13 on matrix factorizations of locally free sheaves and then show how a theorem of Orlov [26] relates these to affine complete intersections. 
2.1. Matrix factorizations of locally free sheaves. Throughout $X$ will denote a Noetherian separated scheme and $\mathcal{L}$ a line bundle on $X$. To simplify notation, even if $\mathcal{L}$ is not very ample, for a quasi-coherent sheaf $\mathcal{G}$ (or a complex of such) on $X$ and integer $n$, we will write $\mathcal{G}(n)$ for $\mathcal{G} \otimes_{\mathcal{O}_{X}} \mathcal{L}^{\otimes n}$, where $\mathcal{L}^{\otimes-n}:=\mathcal{H}_{\mathrm{om}_{\mathcal{O}_{X}}}\left(\mathcal{L}^{\otimes n}, \mathcal{O}_{X}\right)$ for $n \geq 1$. (Here, and elsewhere, Hom denotes the sheaf-Hom.) In particular, $\mathcal{O}(1)=\mathcal{L}$. Similarly, if $f$ is a morphism of (complexes of) quasi-coherent sheaves, then $f(1)=f \otimes i d_{\mathcal{L}}$.

Definition 2.1. Let $W$ be a global section of $\mathcal{L}$. A matrix factorization $\mathbb{E}=$ $\left(\mathcal{E}_{1} \stackrel{e_{1}}{\longrightarrow} \mathcal{E}_{0} \stackrel{e_{0}}{\longrightarrow} \mathcal{E}_{1}(1)\right)$ of the triple $(X, \mathcal{L}, W)$ consists of a pair of locally free coherent sheaves $\mathcal{E}_{1}, \mathcal{E}_{0}$ on $X$ and morphisms $e_{1}: \mathcal{E}_{1} \rightarrow \mathcal{E}_{0}$ and $e_{0}: \mathcal{E}_{0} \rightarrow \mathcal{E}_{1}(1)$ such that $e_{0} \circ e_{1}$ and $e_{1}(1) \circ e_{0}$ are multiplication by $W$. A strict morphism of matrix factorizations from $\left(\mathcal{E}_{1} \rightarrow \mathcal{E}_{0} \rightarrow \mathcal{E}_{1}(1)\right)$ to $\left(\mathcal{F}_{1} \rightarrow \mathcal{F}_{0} \rightarrow \mathcal{F}_{1}(1)\right)$ is a pair of maps $\mathcal{E}_{0} \rightarrow \mathcal{F}_{0}, \mathcal{E}_{1} \rightarrow \mathcal{F}_{1}$ causing the evident squares to commute. Matrix factorizations and strict morphisms of such form a category which we write $M F(X, \mathcal{L}, W)$ or just $M F$ for short.

The previous definition first appeared in 28 . The category $M F(X, \mathcal{L}, W)$ is made into an exact category in the sense of Quillen [30] by declaring a sequence of strict maps to be exact if it is so in both degrees.

Definition 2.2. A twisted periodic complex of locally free coherent sheaves for $(X, \mathcal{L})$ is a chain complex $\mathcal{C}$ of locally free coherent sheaves on $X$ together with a specified isomorphism $\alpha: \mathcal{C}[2] \cong \mathcal{C}(1)$, where we use the convention that $\mathcal{C}[2]^{i}=$ $\mathcal{C}^{i+2}$. The category $\operatorname{TPC}(X, \mathcal{L})$ has twisted periodic complexes as objects, and a morphism is a chain map that commutes with the isomorphisms in the evident sense. There is an equivalence

$$
\operatorname{TPC}(X, \mathcal{L}) \cong M F(X, \mathcal{L}, 0)
$$

given by sending $(\mathcal{C}, \alpha)$ to $\mathcal{C}^{-1} \stackrel{d}{\rightarrow} \mathcal{C}^{0} \stackrel{\alpha^{-1} \circ d}{\longrightarrow} \mathcal{C}^{-1}(1)$.

The motivating example of a twisted periodic complex is the following:

Definition 2.3. Let $\mathbb{E}=\left(\mathcal{E}_{1} \stackrel{e_{1}}{\longrightarrow} \mathcal{E}_{0} \stackrel{e_{0}}{\longrightarrow} \mathcal{E}_{1}(1)\right)$ and $\mathbb{F}=\left(\mathcal{F}_{1} \stackrel{f_{1}}{\longrightarrow} \mathcal{F}_{0} \stackrel{f_{0}}{\longrightarrow} \mathcal{F}_{1}(1)\right)$ be matrix factorizations of $(X, \mathcal{L}, W)$. We define the mapping complex of $\mathbb{E}$ and $\mathbb{F}$, written $\mathcal{H}_{\mathrm{MF}}(\mathbb{E}, \mathbb{F})$, to be the following twisted periodic complex of locally free sheaves:

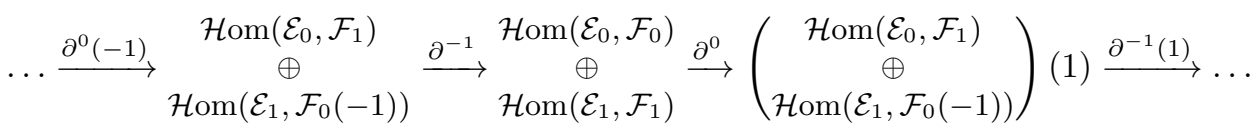

Here $\mathcal{H o m}\left(\mathcal{E}_{0}, \mathcal{F}_{0}\right) \oplus \mathcal{H o m}\left(\mathcal{E}_{1}, \mathcal{F}_{1}\right)$ lies in degree 0 , and the differentials are given by

$$
\partial^{-1}=\left[\begin{array}{cc}
\left(f_{1}\right)_{*} & -e_{0}^{*} \\
-e_{1}^{*} & \left(f_{0}\right)_{*}
\end{array}\right] \quad \text { and } \quad \partial^{0}=\left[\begin{array}{cc}
\left(f_{0}\right)_{*} & e_{0}^{*} \\
e_{1}^{*} & \left(f_{1}\right)_{*}
\end{array}\right],
$$

using the canonical isomorphisms

$$
\mathcal{H} \mathrm{om}\left(\mathcal{E}_{i}, \mathcal{F}_{j}(1)\right) \cong \mathcal{H} \operatorname{om}\left(\mathcal{E}_{i}, \mathcal{F}_{j}\right)(1) \cong \mathcal{H} \operatorname{om}\left(\mathcal{E}_{i}(-1), \mathcal{F}_{j}\right)
$$

and

$$
\left(\begin{array}{c}
\mathcal{H o m}\left(\mathcal{E}_{0}, \mathcal{F}_{1}\right) \\
\oplus \\
\mathcal{H} \operatorname{om}\left(\mathcal{E}_{1}, \mathcal{F}_{0}(-1)\right)
\end{array}\right)(1) \cong \begin{gathered}
\mathcal{H o m}\left(\mathcal{E}_{0}, \mathcal{F}_{1}(1)\right) \\
\oplus \\
\mathcal{H o m}\left(\mathcal{E}_{1}, \mathcal{F}_{0}\right)
\end{gathered}
$$


One checks that $\partial^{0} \circ \partial^{-1}$ and $\partial^{-1}(1) \circ \partial^{0}$ are both zero, and hence $\mathcal{H o m}_{\mathrm{MF}}(\mathbb{E}, \mathbb{F})$ is in fact a twisted periodic complex.

Note that there is an isomorphism

$$
\operatorname{Hom}_{M F}(\mathbb{E}, \mathbb{F}) \cong Z^{0}\left(\Gamma\left(X, \mathcal{H} \operatorname{om}_{\mathrm{MF}}(\mathbb{E}, \mathbb{F})\right)\right),
$$

where $\Gamma\left(X, \mathcal{H} \mathrm{om}_{\mathrm{MF}}(\mathbb{E}, \mathbb{F})\right)$ is the complex of abelian groups obtained by applying the global sections functor degree-wise to $\mathcal{H}_{\mathrm{om}}(\mathbb{E}, \mathbb{F})$, and $Z^{0}$ denotes the cycles in degree zero.

Following [24,28] we define the homotopy category associated to $M F(X, \mathcal{L}, W)$, written $[M F(X, \mathcal{L}, W)]$ or just $[M F]$, as follows. First, one defines the "naive homotopy category", written $[M F]_{\text {naive }}$, to have Hom-sets

$$
\operatorname{Hom}_{[M F]_{\text {naive }}}(\mathbb{E}, \mathbb{F})=\mathrm{H}^{0}\left(\Gamma\left(X, \mathcal{H} \operatorname{om}_{\mathrm{MF}}(\mathbb{E}, \mathbb{F})\right)\right) .
$$

Thus "homotopic" morphisms in $M F$ are identified. The category $[M F]_{\text {naive }}$ is triangulated with shift functor and triangles as given in e.g. [13, 2.5]. An object $\mathbb{E}$ of $[M F]_{\text {naive }}$ is locally contractible if for each $x \in X$ the evident localization at $x$, written $\mathbb{E}_{x}$, is isomorphic to zero in

$$
\left[M F\left(\operatorname{Spec} \mathcal{O}_{X, x}, \mathcal{L}_{x}, W_{x}\right)\right]_{\text {naive }} .
$$

The set of such objects forms a thick subcategory. The homotopy category $[M F]$ is the Verdier quotient

$$
[M F(X, \mathcal{L}, W)]=\frac{[M F(X, \mathcal{L}, W)]_{\text {naive }}}{\text { locally contractible objects }} .
$$

We developed in [13] a more explicit description of the Hom-sets of $[M F]$ when $X$ is projective over a Noetherian ring. Recall that a scheme $X$ is projective over a ring $Q$ if there is a closed embedding $j: X \hookrightarrow \mathbb{P}_{Q}^{m}$ for some $m \geq 0$. In this case, we say that $\mathcal{O}_{X}(1):=j^{*} \mathcal{O}_{\mathbb{P}_{Q}^{m}}(1)$ is the corresponding very ample line bundle on $X$.

To describe the construction, we first make a fixed choice of a finite affine open cover $\mathcal{U}=\left\{U_{1}, \ldots, U_{m}\right\}$ of $X$, and for any quasi-coherent sheaf $\mathcal{F}$ on $X$, let $\Gamma(\mathcal{U}, \mathcal{F})$ denote the usual Cech construction. Since $X$ is separated, the cohomology of the complex $\Gamma(\mathcal{U}, \mathcal{F})$ gives the sheaf cohomology of $\mathcal{F}$. We define $\Gamma\left(\mathcal{U}, \mathcal{H} \operatorname{om}_{\mathrm{MF}}(\mathbb{E}, \mathbb{F})\right)$ to be the total complex associated to the bicomplex

$$
0 \rightarrow \bigoplus_{i} \Gamma\left(U_{i}, \mathcal{H} \operatorname{om}_{\mathrm{MF}}(\mathbb{E}, \mathbb{F})\right) \rightarrow \bigoplus_{i<j} \Gamma\left(U_{i} \cap U_{i}, \mathcal{H} \mathrm{om}_{\mathrm{MF}}(\mathbb{E}, \mathbb{F})\right) \rightarrow \cdots
$$

given by applying the Cech construction degree-wise. If $\mathbb{G}$ is another matrix factorization, there is an evident morphism of chain complexes

$$
\Gamma\left(\mathcal{U}, \mathcal{H} \mathrm{om}_{\mathrm{MF}}(\mathbb{E}, \mathbb{F})\right) \otimes \Gamma\left(\mathcal{U}, \mathcal{H} \mathrm{om}_{\mathrm{MF}}(\mathbb{F}, \mathbb{G})\right) \rightarrow \Gamma\left(\mathcal{U}, \mathcal{H} \mathrm{om}_{\mathrm{MF}}(\mathbb{E}, \mathbb{G})\right)
$$

which is associative and unital.

We set

$$
\mathbb{H}^{q}\left(X, \mathcal{H} \mathrm{om}_{\mathrm{MF}}(\mathbb{E}, \mathbb{F})\right)=\mathrm{H}^{q}\left(\Gamma\left(\mathcal{U}, \mathcal{H} \mathrm{om}_{\mathrm{MF}}(\mathbb{E}, \mathbb{F})\right)\right)
$$

and define the category $[M F(X, \mathcal{L}, W)]_{\mathbb{H}}$ to have the same objects as $M F(X, \mathcal{L}, W)$ with morphisms

$$
\operatorname{Hom}_{[M F]_{\mathbb{H}}}(\mathbb{E}, \mathbb{F}):=\mathbb{H}^{0}\left(X, \mathcal{H} \operatorname{om}_{\mathrm{MF}}(\mathbb{E}, \mathbb{F})\right) .
$$

The composition maps in this category are the maps in homology induced by (2.4). 
There is a functor $[M F]_{\text {naive }} \rightarrow[M F]_{\mathbb{H}}$ that is the identity on objects. The maps on morphisms are given by the canonical maps

$$
\mathrm{H}^{0}\left(\Gamma\left(X, \mathcal{H} \operatorname{om}_{\mathrm{MF}}(\mathbb{E}, \mathbb{F})\right)\right) \rightarrow \mathbb{H}^{0}\left(X, \mathcal{H} \mathrm{om}_{\mathrm{MF}}(\mathbb{E}, \mathbb{F})\right) .
$$

This functor induces a functor $[M F] \rightarrow[M F]_{\mathbb{H}}$ by $[13,3.6]$.

Theorem 2.5 ([13, Theorem 4.2]). Let $X$ be a scheme that is projective over a Noetherian ring $Q$ and $\mathcal{L}=\mathcal{O}_{X}(1)$ be the corresponding very ample line bundle on $X$. For any global section $W$ of $\mathcal{L}$, the functor

$$
[M F(X, \mathcal{L}, W)] \rightarrow[M F(X, \mathcal{L}, W)]_{\mathbb{H}}
$$

is an equivalence.

The category $[M F(X, \mathcal{L}, W)]_{\mathbb{H}}$ is similar to and inspired by Shipman's category of matrix factorizations defined in [31. In the rest of the paper $X$ will always be assumed projective over an affine scheme and we write $[M F]$ for $[M F]_{\mathbb{H}}$.

2.2. Singularity category. The singularity category of a scheme $Z$ is the Verdier quotient

$$
\mathrm{D}_{\mathrm{sg}}(Z):=\mathrm{D}^{\mathrm{b}}(Z) / \operatorname{Perf}(Z)
$$

where $\mathrm{D}^{\mathrm{b}}(Z)$ is the bounded derived category of coherent sheaves on $Z$ and $\operatorname{Perf}(Z)$ is the full subcategory consisting of perfect complexes - i.e., those complexes that are locally quasi-isomorphic to bounded complexes of free modules of finite rank. This construction was introduced by Buchweitz [11] in the case when $Z$ is affine and rediscovered by Orlov [25].

We need the following generalization:

Definition 2.6. Let $i: Z \hookrightarrow X$ be a closed immersion of finite flat dimension. An object $\mathcal{F}$ in $\mathrm{D}^{\mathrm{b}}(Z)$ is relatively perfect on $Z$ if $i_{*} \mathcal{F}$ is perfect on $X$. We write $\operatorname{RPerf}(Z \hookrightarrow X)$ for the full subcategory of $\mathrm{D}^{\mathrm{b}}(Z)$ whose objects are relatively perfect on $X$.

Since $i$ has finite flat dimension, $\operatorname{Perf}(Z)$ is a thick subcategory of $\operatorname{RPerf}(Z \hookrightarrow X)$. We define the relative singularity category of $i$ to be the Verdier quotient

The canonical functor

$$
\mathrm{D}_{\mathrm{sg}}^{\mathrm{rel}}(Z \hookrightarrow X):=\frac{\operatorname{RPerf}(Z \hookrightarrow X)}{\operatorname{Perf}(Z)} .
$$

$$
\mathrm{D}_{\mathrm{sg}}^{\mathrm{rel}}(Z \hookrightarrow X) \rightarrow \mathrm{D}_{\mathrm{sg}}(Z)
$$

is fully faithful and we thus identify $\mathrm{D}_{\mathrm{sg}}^{\text {rel }}(Z \hookrightarrow X)$ with a full subcategory of $\mathrm{D}_{\mathrm{sg}}(Z)$. (A different definition of "relative singularity category" is given by Positselski in [29]. There is a fully faithful functor from the version given here to Positselski's version, but in general the two need not coincide; see [13, 6.9].)

If $X=\operatorname{Spec} Q$ is affine, so that $Z=\operatorname{Spec} R$ where $R=Q / I$, we write $\mathrm{D}_{\mathrm{sg}}^{\text {rel }}(Q \rightarrow R)$ for $\mathrm{D}_{\mathrm{sg}}^{\text {rel }}(\operatorname{Spec} R \hookrightarrow \operatorname{Spec} Q)$. In this case, a finitely generated $R$-module $M$ is in $\mathrm{D}_{\mathrm{sg}}^{\mathrm{rel}}(Q \rightarrow R)$ if and only if it has finite projective dimension as a $Q$-module.

Recall that $\mathcal{L}$ is a line bundle on $X$ and $W$ is a global section of $\mathcal{L}$. We now set $Z \hookrightarrow X$ to be the zero subscheme of $W$ (i.e., the subscheme with ideal sheaf given as the image of the map $\left.W^{*}: \mathcal{L}^{*} \rightarrow \mathcal{O}_{X}\right)$.

For an object $\mathbb{E}=\left(\mathcal{E}_{1} \stackrel{e_{1}}{\rightarrow} \mathcal{E}_{0} \stackrel{e_{0}}{\longrightarrow} \mathcal{E}_{1}(1)\right)$ of $M F(X, \mathcal{L}, W)$, we define the cokernel of $\mathbb{E}$, written coker $(\mathbb{E})$, to be coker $\left(e_{1}\right)$. Multiplication by $W$ on $\operatorname{coker}\left(e_{1}\right)$ is zero, 
and so we regard coker $\mathbb{E}$ as a coherent sheaf on $Z$. When $W$ is a regular, i.e. the map $\mathcal{O}_{X} \stackrel{W}{\longrightarrow} \mathcal{L}$ is injective, there is a finite resolution of $i_{*} \operatorname{coker}(\mathbb{E})$ on $X$ by locally free sheaves:

$$
0 \rightarrow \mathcal{E}_{1} \stackrel{e_{1}}{\rightarrow} \mathcal{E}_{0} \rightarrow i_{*} \operatorname{coker}(\mathbb{E}) \rightarrow 0,
$$

and hence $i_{*} \operatorname{coker}(\mathbb{E})$ is perfect on $X$. Thus the image of coker $(\mathbb{E})$ in $\mathrm{D}_{\mathrm{sg}}(Z)$ is in $\mathrm{D}_{\mathrm{sg}}^{\mathrm{rel}}(Z \hookrightarrow X)$. This assignment is clearly natural and by [28, 3.12] there is an induced triangulated functor

$$
\begin{gathered}
\text { coker }:[M F(X, \mathcal{L}, W)] \rightarrow \mathrm{D}_{\mathrm{sg}}^{\text {rel }}(Z \hookrightarrow X), \\
\mathbb{E} \mapsto \operatorname{coker}(\mathbb{E}) .
\end{gathered}
$$

The following was first proved by Polishchuk and Vaintrob in the case when $X$ is regular, see [28, 3.14], and there are analogues in [22,24, 29]. The version below is $[13,6.3]$.

Theorem 2.7. Let $X$ be a scheme that is projective over a Noetherian ring of finite Krull dimension, $\mathcal{L}=\mathcal{O}_{X}(1)$ be the corresponding very ample line bundle, and $W$ be a regular global section of $\mathcal{L}$. Define $i: Z \hookrightarrow X$ to be the zero subscheme of $W$. Then the triangulated functor

$$
\text { coker : }[M F(X, \mathcal{L}, W)] \rightarrow \mathrm{D}_{\mathrm{sg}}^{\mathrm{rel}}(Z \hookrightarrow X)
$$

is an equivalence. In particular, if $X$ is regular, there is an equivalence of triangulated categories

$$
\text { coker : }\left[M F(X, \mathcal{L}, W) \stackrel{\cong}{\longrightarrow} \mathrm{D}_{\mathrm{sg}}(Z)\right. \text {. }
$$

Corollary 2.8. Let $X, Z$ and $i$ be as in Theorem 2.7. If $\mathcal{M}$ is any object of $\mathrm{D}_{\mathrm{sg}}^{\mathrm{rel}}(Z \hookrightarrow X)$, then there is a coherent sheaf $\mathcal{F}$ on $Z$ and an isomorphism $\mathcal{M} \cong \mathcal{F}$ in the category $\mathrm{D}_{\mathrm{sg}}^{\mathrm{rel}}(Z \hookrightarrow X)$. In fact, $\mathcal{F}$ may be chosen so that $i_{*}(\mathcal{F})$ admits a resolution of length one by locally free coherent sheaves on $X$.

Proof. Let $\mathbb{E}$ be the image of $\mathcal{M}$ under some inverse of coker. Then $\mathcal{M} \cong$ coker $\mathbb{E}$, and coker $\mathbb{E}$ is a coherent sheaf that admits a resolution of length one by locally free coherent sheaves.

2.3. Orlov's Theorem. We now fix $Q$ to be a commutative Noetherian ring of finite Krull dimension, $\mathbf{f}=\left(f_{1}, \ldots, f_{c}\right)$ a $Q$-regular sequence, and $R=Q /(\mathbf{f})$. Set

$$
\mathbb{P}_{Q}^{\mathrm{c}-1}=\operatorname{Proj}\left(Q\left[T_{1}, \ldots, T_{c}\right]\right) \quad \text { and } \quad \mathbb{P}_{R}^{\mathrm{c}-1}=\operatorname{Proj}\left(R\left[T_{1}, \ldots, T_{c}\right]\right),
$$

where each $T_{i}$ has degree 1 . Define $W=f_{1} T_{1}+\ldots+f_{c} T_{c} \in \Gamma\left(\mathbb{P}_{Q}^{c-1}, \mathcal{O}_{\mathbb{P}_{Q}^{c-1}}(1)\right)$ and

$$
Y=\operatorname{Proj}\left(Q\left[T_{1}, \ldots, T_{c}\right] / W\right) \hookrightarrow \mathbb{P}_{Q}^{\mathrm{c}-1} .
$$

The natural surjection $Q \rightarrow R$ induces an inclusion $\delta: \mathbb{P}_{R}^{\mathrm{c}-1} \hookrightarrow \mathbb{P}_{Q}^{\mathrm{c}-1}$. This is locally a complete intersection of codimension $c-1$ and factors through the map $\gamma: Y \hookrightarrow \mathbb{P}_{Q}^{c-1}$. We have a commutative diagram of schemes

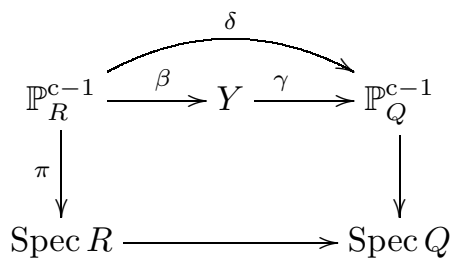


where the vertical arrows are the canonical proper maps and each horizontal arrow is locally a complete intersection and thus has finite flat dimension. Since $\beta$ is a finite map, there is a right adjoint to $\beta_{*}$, see [18, III.6], which we write as $\beta^{b}$ : $\mathrm{D}^{\mathrm{b}}(Y) \rightarrow \mathrm{D}^{\mathrm{b}}\left(\mathbb{P}_{R}^{\mathrm{c}-1}\right)$.

The following was proved by Orlov in [26, Theorem 2.1] under the added assumption that $Q$ is regular and equicharacteristic, i.e. contains a field. A proof of the version below may be found in the appendix; see Theorem A.4.

Theorem 2.10. The functor $\mathbf{R} \pi_{*} \beta^{\mathrm{b}}: \mathrm{D}^{\mathrm{b}}(Y) \rightarrow \mathrm{D}^{\mathrm{b}}(R)$ induces an equivalence of triangulated categories

$$
\Phi: \mathrm{D}_{\mathrm{sg}}^{\mathrm{rel}}\left(Y \subset \mathbb{P}_{Q}^{c-1}\right) \stackrel{\cong}{\longrightarrow} \mathrm{D}_{\mathrm{sg}}^{\mathrm{rel}}(Q \rightarrow R) .
$$

In particular, if $Q$ is regular, we have an equivalence

$$
\Phi: \mathrm{D}_{\mathrm{sg}}(Y) \cong \mathrm{D}_{\mathrm{sg}}(R) .
$$

Proof. In the notation of Theorem A.4, let $S=\operatorname{Spec} Q$, let $\mathcal{E}$ be a free $Q$-module of rank $c$, and let $s=\left(f_{1}, \ldots, f_{c}\right) \in \Gamma(S, \mathcal{E})=Q^{c}$.

As an immediate consequence of Theorems 2.7 and 2.10 we have:

Corollary 2.11. There is an equivalence of triangulated categories

$$
\Psi=\Phi \circ \text { coker }:\left[M F\left(\mathbb{P}_{Q}^{c-1}, \mathcal{O}(1), W\right)\right] \stackrel{\cong}{\longrightarrow} \mathrm{D}_{\mathrm{sg}}^{\text {rel }}(Q \rightarrow R)
$$

such that

$$
\Psi(\mathbb{E})=\mathbf{R} \pi_{*} \beta^{\mathrm{b}} \text { coker } \mathbb{E} \in \mathrm{D}_{\mathrm{sg}}(R) .
$$

In particular, if $Q$ is regular, we have an equivalence of triangulated categories

$$
\Psi:\left[M F\left(\mathbb{P}_{Q}^{c-1}, \mathcal{O}(1), W\right)\right] \stackrel{\cong}{\longrightarrow} \mathrm{D}_{\mathrm{sg}}(R) .
$$

The inverse equivalence $\mathrm{D}_{\mathrm{sg}}^{\mathrm{rel}}(Q \rightarrow R) \rightarrow \mathrm{D}_{\mathrm{sg}}^{\mathrm{rel}}\left(Y \subset \mathbb{P}_{Q}^{\mathrm{c}-1}\right)$ is induced by the functor $\mathbf{R} \beta_{*} \circ \mathbf{L} \pi^{*} \cong \beta_{*} \circ \pi^{*}$, but the inverse of coker : $[M F] \stackrel{\cong}{\longrightarrow} \mathrm{D}_{\mathrm{sg}}^{\text {rel }}\left(Y \subset \mathbb{P}_{Q}^{\mathrm{c}-1}\right)$ requires making choices, and hence so does an inverse equivalence to $\Psi$. We fix one such inverse equivalence $\Psi^{-1}: \mathrm{D}_{\mathrm{sg}}^{\mathrm{rel}}(Q \rightarrow R) \rightarrow[M F]$, and for $M \in \mathrm{D}_{\mathrm{sg}}^{\mathrm{rel}}(Q \rightarrow R)$ we write $\mathbb{E}_{M} \in[M F]$ for $\Psi^{-1}(M)$. Recall that specifying the inverse $\Psi^{-1}$ amounts to picking, for each object $M$ of $\mathrm{D}_{\mathrm{sg}}^{\mathrm{rel}}(Q \rightarrow R)$, a matrix factorization $\mathbb{E}_{M}$ together with an isomorphism $\Psi\left(\mathbb{E}_{M}\right) \stackrel{\cong}{\longrightarrow} M$ in $\mathrm{D}_{\text {sg }}^{\text {rel }}(Q \rightarrow R)$. In Section [6 we give one explicit way of constructing $\mathbb{E}_{M}$ using a system of higher homotopies on a $Q$-free resolution of $M$.

Definition 2.12. Let $A$ be any commutative Noetherian ring and let $M$ and $N$ be complexes of $A$-modules with bounded finitely generated homology. For $q \in \mathbb{Z}$, the $q$-th stable Ext-module of $M$ and $N$ is

$$
\widehat{\operatorname{Ext}}_{A}^{q}(M, N):=\operatorname{Hom}_{\mathrm{D}_{\mathrm{sg}}(A)}(M, N[q]) \text {. }
$$

The definition above first appeared in [11 where the $\operatorname{ring} A$ was assumed to be Gorenstein. We show in Appendix B that, as in [11, the stable Ext-modules may be computed using a complete resolution of $M$ when such a resolution exists.

Note that for all $q \in \mathbb{Z}$ there is a natural map

$$
\operatorname{Ext}_{A}^{q}(M, N) \rightarrow \widehat{\operatorname{Ext}}_{A}^{q}(M, N),
$$

induced by the triangulated functor $\mathrm{D}^{\mathrm{b}}(A) \rightarrow \mathrm{D}_{\mathrm{sg}}(A)$. 
The next result follows immediately from Corollary 2.11 and the definition of morphisms in $[M F]$.

Corollary 2.14. Let $M$ and $N$ be objects of $\mathrm{D}_{\mathrm{sg}}^{\mathrm{rel}}(Q \rightarrow R)$. For all $q \in \mathbb{Z}$ there is an isomorphism, natural in $M$ and $N$,

$$
\widehat{\operatorname{Ext}}_{R}^{q}(M, N) \cong \mathbb{H}^{q}\left(\mathbb{P}_{Q}^{c-1}, \mathcal{H} \operatorname{om}_{\mathrm{MF}}\left(\mathbb{E}_{M}, \mathbb{E}_{N}\right)\right) .
$$

In particular, if $Q$ is regular, such an isomorphism holds for all finitely generated $R$-modules.

Fix an object $\mathbb{E}=\left(\mathcal{E}_{1} \stackrel{e_{1}}{\rightarrow} \mathcal{E}_{0} \stackrel{e_{0}}{\longrightarrow} \mathcal{E}_{1}(1)\right)$ of $M F\left(\mathbb{P}_{Q}^{\mathrm{c}-1}, \mathcal{O}(1), W\right)$. We write $\gamma^{*} \mathbb{E}$ for the following complex of locally free coherent sheaves on $Y$ :

$$
\cdots \rightarrow \gamma^{*} \mathcal{E}_{0}(-1) \stackrel{\gamma^{*} e_{0}(-1)}{\longrightarrow} \gamma^{*} \mathcal{E}_{1} \stackrel{\gamma^{*} e_{1}}{\longrightarrow} \gamma^{*} \mathcal{E}_{0} \stackrel{\gamma^{*} e_{0}}{\longrightarrow} \gamma^{*} \mathcal{E}_{1}(1) \stackrel{\gamma^{*} e_{1}(1)}{\longrightarrow} \gamma^{*} \mathcal{E}_{0}(1) \rightarrow \cdots,
$$

where $\gamma: Y \hookrightarrow \mathbb{P}_{Q}^{\mathrm{c}-1}$ is the natural inclusion. This is a complex since multiplication by $W$ is the zero map on $Y$.

The following result is an analogue of Corollary 2.14 which relaxes the assumption that $N$ is perfect over $Q$.

Proposition 2.16. Let $M$ be an object in $\mathrm{D}_{\mathrm{sg}}^{\mathrm{rel}}(Q \rightarrow R)$ and let $N$ be any object in $\mathrm{D}_{\mathrm{sg}}(R)$. Set $\mathcal{N}$ to be the image of $\beta_{*} \pi^{*} N$ in $\mathrm{D}_{\mathrm{sg}}(Y)$. For all $q \in \mathbb{Z}$ there are isomorphisms that are natural in both arguments:

$$
\begin{aligned}
\widehat{\operatorname{Ext}}_{R}^{q}(M, N) & \cong \operatorname{Hom}_{\mathrm{D}_{\mathrm{sg}}(R)}\left(\Psi\left(\mathbb{E}_{M}\right), N[q]\right) \\
& \cong \operatorname{Hom}_{\mathrm{D}_{\mathrm{sg}}(Y)}\left(\operatorname{coker} \mathbb{E}_{M}, \mathcal{N}[q]\right) \\
& \cong \mathbb{H}^{q}\left(Y, \mathcal{H} \operatorname{om}_{\mathcal{O}_{Y}}\left(\gamma^{*} \mathbb{E}_{M}, \mathcal{N}\right)\right) .
\end{aligned}
$$

Proof. By definition $\Psi\left(\mathbb{E}_{M}\right)=\mathbf{R} \pi_{*} \beta^{b} \operatorname{coker}\left(\mathbb{E}_{M}\right) \cong M$ in $\mathrm{D}_{\mathrm{sg}}(R)$. This gives the first isomorphism in (2.17).

The functor $\beta_{*} \pi^{*}$ induces an equivalence $\mathrm{D}_{\mathrm{sg}}^{\text {rel }}(Q \rightarrow R) \rightarrow \mathrm{D}_{\mathrm{sg}}^{\text {rel }}(Y \hookrightarrow X)$. This is an inverse to $\mathbf{R} \pi_{*} \beta^{\text {b }}$, so the natural map $\beta_{*} \pi^{*} \mathbf{R} \pi_{*} \beta^{\text {b }} \operatorname{coker}\left(\mathbb{E}_{M}\right) \rightarrow \operatorname{coker}\left(\mathbb{E}_{M}\right)$ is an isomorphism in $\mathrm{D}_{\mathrm{sg}}(Y)$. This gives

$$
\begin{aligned}
\operatorname{Hom}_{\mathrm{D}_{\mathrm{sg}}(R)}\left(\Psi\left(\mathbb{E}_{M}\right)[-q], N\right) & =\operatorname{Hom}_{\mathrm{D}_{\mathrm{sg}}(R)}\left(\mathbf{R} \pi_{*} \beta^{b} \operatorname{coker}\left(\mathbb{E}_{M}\right)[-q], N\right) \\
& \cong \operatorname{Hom}_{\mathrm{D}_{\mathrm{sg}}(Y)}\left(\beta_{*} \pi^{*} \mathbf{R} \pi_{*} \beta^{b} \operatorname{coker}\left(\mathbb{E}_{M}\right)[-q], \mathcal{N}\right) \\
& \cong \operatorname{Hom}_{\mathrm{D}_{\mathrm{sg}}(Y)}\left(\operatorname{coker}\left(\mathbb{E}_{M}\right)[-q], \mathcal{N}\right),
\end{aligned}
$$

which is the second isomorphism in (2.17). The third isomorphism is due to 13 , $5.10]$.

\section{EISENBUD OPERATORS}

We remain under the assumptions of $\$ 2.3$. For each matrix factorization $\mathbb{E}$ and any $i=1, \ldots, c$, multiplication by $T_{i} \in \Gamma\left(\mathbb{P}_{Q}^{c-1}, \mathcal{O}_{\mathbb{P}_{Q}^{c-1}}(1)\right)$ defines a map

$$
T_{i}^{\mathbb{E}}: \mathbb{E} \rightarrow \mathbb{E}[2]
$$


given by

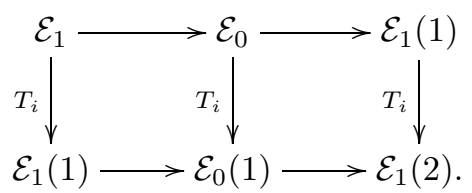

These maps determine natural transformations

$$
T_{i}: i d_{[M F]} \rightarrow(-)[2]_{[M F]} \quad \text { for } \quad i=1, \ldots, c
$$

from the identity functor on $[M F]$ to the functor which sends $\mathbb{E}$ to $\mathbb{E}[2]$.

On the other hand, there is a family of natural transformations $t_{i}^{\prime}: i d_{\mathrm{D}^{\mathrm{b}}(R)} \rightarrow$ $(-)[2]_{\mathrm{D}^{\mathrm{b}}(R)}$, for $i=1, \ldots, c$, given by the Eisenbud operators [15; the construction of these maps is recalled in 4.1 below. These descend to natural transformations

$$
t_{i}: i d_{\mathrm{D}_{\mathrm{sg}}(R)} \rightarrow(-)[2]_{\mathrm{D}_{\mathrm{sg}}(R)} \text { for } \quad i=1, \ldots, c .
$$

One expects that these two natural transformations coincide via the equivalence

$$
\Psi:[M F] \stackrel{\cong}{\longrightarrow} \mathrm{D}_{\mathrm{sg}}^{\mathrm{rel}}(Q \rightarrow R)
$$

of Corollary 2.11, and this is precisely the content of the following theorem.

Theorem 3.2. For each object $\mathbb{E}$ of $[M F]$ there is a commutative diagram in $\mathrm{D}_{\mathrm{sg}}(R)$,

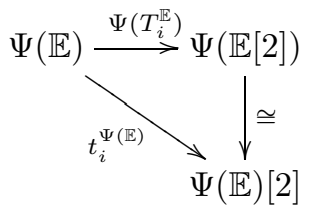

where the vertical map is the canonical isomorphism associated to the triangulated functor $\Psi$.

Our proof of this theorem requires the development of additional machinery, and is contained in Section 4. In this section we explore several consequences of the theorem. The first is the following:

Corollary 3.3. Let $M$ and $N$ be objects of $\mathrm{D}_{\mathrm{sg}}^{\mathrm{rel}}(Q \rightarrow R)$. The diagram

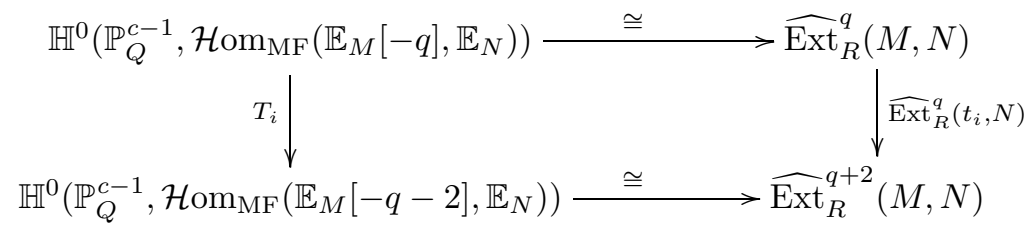

commutes for $i=1, \ldots, c$, where the horizontal isomorphisms are given by Corollary 2.14, the left vertical map is $\mathbb{H}^{0}\left(\mathbb{P}_{Q}^{c-1}, T_{i}\right)$, where $T_{i}$ is multiplication by $T_{i} \in$ $\Gamma\left(\mathbb{P}_{Q}^{c-1}, \mathcal{O}(1)\right)$ on the complex of coherent sheaves $\mathcal{H o m}_{\mathrm{MF}}\left(\mathbb{E}_{M}[-q], \mathbb{E}_{N}\right)$, composed with the natural isomorphism

$\mathcal{H o m}_{\mathrm{MF}}\left(\mathbb{E}_{M}[-q], \mathbb{E}_{N}\right)(1) \cong \mathcal{H o m}_{\mathrm{MF}}\left(\mathbb{E}_{M}[-q], \mathbb{E}_{N}\right)[2] \cong \mathcal{H} \mathrm{om}_{\mathrm{MF}}\left(\mathbb{E}_{M}[-q-2], \mathbb{E}_{N}\right)$, and $t_{i}$ is the $i$-th Eisenbud operator. 
Proof. Let $T_{i}: \mathbb{E}[-q-2] \rightarrow \mathbb{E}[-q]$ be the map defined in (3.1). Consider the diagram

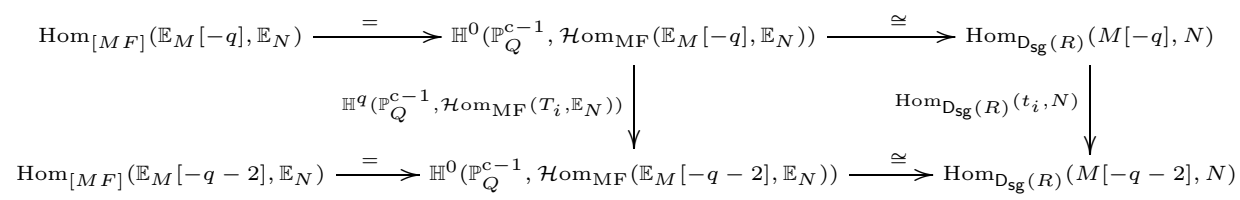

where the horizontal maps are induced by the functor $\Psi$. The diagram commutes by Theorem 3.2. One checks that the middle vertical map above is equal to the left vertical map in the statement of the corollary.

We wish to extend the previous result by dropping the assumption that $N$ is perfect over $Q$. This is best stated using a "stable Ext sheaf" introduced below. With an eye toward future applications, we work at a level of greater generality than needed presently.

3.1. Stable Ext sheaf. Let $X$ be a scheme that is projective over a Noetherian ring $A$ of finite Krull dimension, $\mathcal{L}=\mathcal{O}(1)$ the associated very ample line bundle, and $W$ a regular global section of $\mathcal{L}$. Let $\gamma: Z \hookrightarrow X$ be the embedding of the zero subscheme of $W$. Under these assumptions, by Theorem 2.7 there is an equivalence

$$
\text { coker : }[M F(X, \mathcal{L}, W)] \stackrel{\cong}{\longrightarrow} \mathrm{D}_{\text {sg }}^{\text {rel }}(Z \hookrightarrow X) \text {. }
$$

We fix an inverse equivalence of coker, and for $\mathcal{M}$ in $\mathrm{D}_{\mathrm{sg}}^{\text {rel }}(Z \hookrightarrow X)$ we let $\mathbb{E}_{\mathcal{M}}$ denote the image of $\mathcal{M}$ under this inverse.

Definition 3.4. Let $X, \mathcal{L}, W$, and $Z$ be as above. For $\mathcal{M}$ in $\mathrm{D}_{\mathrm{sg}}^{\text {rel }}(Z \hookrightarrow X), \mathcal{N}$ a bounded complex of coherent sheaves on $Z$, and an integer $q \in \mathbb{Z}$, define

$$
\widehat{\mathcal{E x t}}_{\mathcal{O}_{Z}}^{q}(\mathcal{M}, \mathcal{N})=\mathcal{H}^{q} \mathcal{H} \operatorname{om}_{\mathcal{O}_{Z}}\left(\gamma^{*} \mathbb{E}_{\mathcal{M}}, \mathcal{N}\right)
$$

where $\gamma^{*} \mathbb{E}_{\mathcal{M}}$ is the complex of locally free coherent sheaves on $Z$ defined in (2.15).

Remark 3.5. If $\mathcal{N}$ is also in $\mathrm{D}_{\mathrm{sg}}^{\text {rel }}(Z \hookrightarrow X)$, then by [13, 5.2.3] there is an isomorphism $\gamma_{*} \mathcal{H}_{\mathrm{om}_{\mathcal{O}_{Z}}}\left(\gamma^{*} \mathbb{E}_{\mathcal{M}}, \mathcal{N}\right) \cong \mathcal{H} \operatorname{om}_{\mathrm{MF}}\left(\mathbb{E}_{M}, \mathbb{E}_{N}\right)$. Thus for each $q \in \mathbb{Z}$ there is an isomorphism

$$
\gamma_{*} \widehat{\mathcal{E x t}}_{\mathcal{O}_{Z}}^{q}(\mathcal{M}, \mathcal{N}) \cong \mathcal{H}^{q} \mathcal{H} \mathrm{om}_{\mathrm{MF}}\left(\mathbb{E}_{M}, \mathbb{E}_{N}\right)
$$

Remark 3.6. When $Z=\operatorname{Spec} A$ is affine, the definition above agrees with the previous definition of stable Ext given in Definition 2.12 by Example B.5 and Lemma B.6.

Lemma 3.7. The rule $(\mathcal{M}, \mathcal{N}) \mapsto \mathcal{H}^{\circ} \mathrm{O}_{\mathcal{O}_{Z}}\left(\gamma^{*} \mathbb{E}_{\mathcal{M}}, \mathcal{N}\right)$ defines a functor from $\mathrm{D}_{\mathrm{sg}}^{\mathrm{rel}}(Z \hookrightarrow X)^{\mathrm{op}} \times \mathrm{D}^{\mathrm{b}}(Z)$ to $\mathrm{D}(\operatorname{coh} Z)$, the unbounded derived category of $\operatorname{coh} Z$. In particular, ${\widehat{\mathcal{E} \mathrm{xt}_{\mathcal{O}_{Z}}^{q}}}^{q}(-,-)$ is a functor from $\mathrm{D}_{\mathrm{sg}}^{\mathrm{rel}}(Z \hookrightarrow X)^{\mathrm{op}} \times \mathrm{D}^{\mathrm{b}}(Z)$ to $\operatorname{coh} Z$.

Proof. Recall $\mathrm{D}(\operatorname{coh} Z)$ may be defined as the category of complexes of coherent sheaves, $\mathcal{C}(\operatorname{coh} Z)$, with quasi-isomorphisms inverted. Then $\mathrm{D}^{\mathrm{b}}(Z)$ is the full subcategory of $\mathrm{D}(\operatorname{coh} Z)$ with objects those complexes with bounded cohomology. For any fixed matrix factorization $\mathbb{E}$, we have a functor from $\mathcal{C}^{b}(Z)$ to $\mathcal{C}(\operatorname{coh} Z)$ given by

$$
\mathcal{N} \mapsto \mathcal{H} \mathrm{om}_{\mathcal{O}_{Z}}\left(\gamma^{*} \mathbb{E}_{M}, \mathcal{N}\right)
$$

Since each component of $\gamma^{*} \mathbb{E}_{M}$ is locally free and $\mathcal{N}$ is bounded, this functor preserves quasi-isomorphisms and hence induces a functor from $\mathrm{D}^{\mathrm{b}}(Z)$ to $\mathrm{D}(Z)$. 
Since the construction is natural for strict morphisms of matrix factorizations, we obtain a functor from $M F(X, \mathcal{L}, W)^{\mathrm{op}} \times \mathrm{D}^{\mathrm{b}}(Z)$ to $\mathrm{D}(\operatorname{coh} Z)$.

If $\mathbb{E} \rightarrow \mathbb{F}$ is locally a homotopy equivalence, then so are $\gamma^{*} \mathbb{E} \rightarrow \gamma^{*} \mathbb{F}$ and

$$
\mathcal{H o m}_{\mathcal{O}_{Z}}\left(\gamma^{*} \mathbb{E}_{M}, \mathcal{N}\right) \rightarrow \mathcal{H o m}_{\mathcal{O}_{Z}}\left(\gamma^{*} \mathbb{E}_{M}, \mathcal{N}\right),
$$

and hence the latter is a quasi-isomorphism. It follows that we get an induced functor

$$
[M F(X, \mathcal{L}, W)]^{\mathrm{op}} \times \mathrm{D}^{\mathrm{b}}(Z) \rightarrow \mathrm{D}(\operatorname{coh} Z) .
$$

Precomposing with the chosen inverse of coker gives the result.

Proposition 3.8. Let $X, \mathcal{L}, W$, and $Z$ be as above. Also, let $\mathcal{M}$ be an object of $\mathrm{D}_{\mathrm{sg}}^{\mathrm{rel}}(Z \hookrightarrow X)$ and $\mathcal{N}$ be an object of $\mathrm{D}^{\mathrm{b}}(Z)$. For all $y \in Z$ and $q \in \mathbb{Z}$ there are isomorphisms

$$
\begin{aligned}
& \widehat{\mathcal{E x t}}_{\mathcal{O}_{z}}^{q}(\mathcal{M}, \mathcal{N}) \cong \widehat{\mathcal{E x t}}_{\mathcal{O}_{z}}^{q+2}(\mathcal{M}, \mathcal{N})(-1) \text { and } \\
& \widehat{\mathcal{E x t}}_{\mathcal{O}_{z}}^{q}(\mathcal{M}, \mathcal{N})_{y} \cong \widehat{\operatorname{Ext}}_{\mathcal{O}_{z, y}}^{q}\left(\mathcal{M}_{y}, \mathcal{N}_{y}\right)
\end{aligned}
$$

that are natural in $\mathcal{M}$ and $\mathcal{N}$. For all $q$, there is a map

$$
\operatorname{Hom}_{\mathrm{D}_{\mathrm{sg}}(Z)}(\mathcal{M}[-q], \mathcal{N}) \cong \mathbb{H}^{q}\left(Z, \mathcal{H} \operatorname{om}_{\mathcal{O}_{Z}}\left(\gamma^{*} \mathbb{E}_{\mathcal{M}}, \mathcal{N}\right)\right) \rightarrow \Gamma\left(Z, \widehat{\mathcal{E}}_{\mathrm{xt}_{\mathcal{O}_{Z}}^{q}}^{q}(\mathcal{M}, \mathcal{N})\right),
$$

that is natural in $\mathcal{M}$ and $\mathcal{N}$. This map is an isomorphism when $q \gg 0$.

Proof. The first isomorphism is due to the fact that $\mathcal{H o m}_{\mathcal{O}_{Z}}\left(\gamma^{*} \mathbb{E}_{\mathcal{M}}, \mathcal{N}\right)$ is a twisted periodic complex; see Definition 2.2 .

For the second isomorphism, we are in the context of Example B.5 which shows that there is a complete resolution $T \rightarrow P \rightarrow \mathcal{M}_{y}$ with $T=\gamma^{*}\left(\mathbb{E}_{\mathcal{M}}\right)_{y}$. It follows from Lemma B.6 that $T$ can be used to compute $\widehat{\operatorname{Ext}}_{\mathcal{O}_{z, y}}\left(\mathcal{M}_{y}, \mathcal{N}_{y}\right)$ and we have

$$
\begin{aligned}
\widehat{\operatorname{Ext}}_{\mathcal{O}_{z, y}}^{q}\left(\mathcal{M}_{y}, \mathcal{N}_{y}\right) & \cong \mathrm{H}^{q}\left(\operatorname{Hom}_{\mathcal{O}_{z, y}}\left(\gamma^{*}\left(\mathbb{E}_{\mathcal{M}}\right)_{y}, \mathcal{N}_{y}\right)\right) \\
& \cong \mathcal{H}^{q}\left(\mathcal{H} \operatorname{om}_{\mathcal{O}_{z}}\left(\gamma^{*}\left(\mathbb{E}_{\mathcal{M}}\right), \mathcal{N}\right)\right)_{y} \\
& =\widehat{\mathcal{E} x t}_{\mathcal{O}_{z}}^{q}(\mathcal{M}, \mathcal{N})_{y} .
\end{aligned}
$$

The last map in the statement of the proposition is an edge map in the spectral sequence

$$
E_{2}^{p, q}=H^{p}\left(Z, \widehat{\mathcal{E}}_{\mathrm{xt}_{\mathcal{O}_{Z}}^{q}}^{q}(\mathcal{M}, \mathcal{N})\right) \Longrightarrow \mathbb{H}^{p+q}\left(Z, \mathcal{H} \operatorname{om}_{\mathcal{O}_{Z}}\left(\gamma^{*} \mathbb{E}_{M}, \mathcal{N}\right)\right) .
$$

By the first isomorphism and the fact that $\mathcal{L}$ is very ample, we obtain

$$
H^{p}\left(Z, \widehat{\mathcal{E}}_{\mathrm{xt}_{\mathcal{O}_{Z}}^{q}}^{q}(\mathcal{M}, \mathcal{N})\right)=0
$$

if $p>0$ and $q \gg 0$ by Serre's Vanishing Theorem, and hence the map is an isomorphism for $q \gg 0$.

The following is the sought-after generalization of Corollary 3.3, which drops the requirement that $N$ be perfect over $Q$. We return to the context and notation of \$2.3.

Corollary 3.9. Let $M$ be an object of $\mathrm{D}_{\mathrm{sg}}^{\mathrm{rel}}(Q \rightarrow R)$ and let $N$ be an object of $\mathrm{D}_{\mathrm{sg}}(R)$. Let $\mathcal{M}$ and $\mathcal{N}$ be the images of $\beta_{*} \pi^{*} M$ and $\beta_{*} \pi^{*} N$, respectively, in $\mathrm{D}_{\mathrm{sg}}(Y)$. For all $q \in \mathbb{Z}$ there is a natural map

$$
\widehat{\operatorname{Ext}}_{R}^{q}(M, N) \rightarrow \Gamma\left(Y,{\widehat{\mathcal{E} \operatorname{xt}_{\mathcal{O}_{Y}}}}^{q}(\mathcal{M}, \mathcal{N})\right)
$$


that makes the following diagram commute:

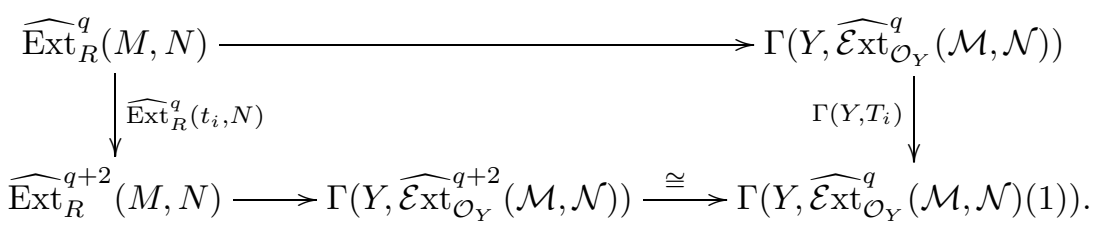

Here $T_{i}$ is multiplication by $T_{i} \in \Gamma\left(Y, \mathcal{O}_{Y}(1)\right)$ on the coherent sheaf $\widehat{\mathcal{E} x t}_{\mathcal{O}_{Y}}^{q}(\mathcal{M}, \mathcal{N})$, the isomorphism on the lower-right is from Proposition 3.8, and $t_{i}$ is the $i$-th Eisenbud operator. Moreover, for $q \gg 0$, the horizontal maps in this diagram are isomorphisms.

Proof. By Proposition 2.16 we have the following string of isomorphisms:

$\operatorname{Hom}_{\mathrm{D}_{\mathrm{sg}}(R)}(M[-q], N) \stackrel{\cong}{\rightarrow} \operatorname{Hom}_{\mathrm{D}_{\mathrm{sg}}(R)}\left(\Psi\left(\mathbb{E}_{M}\right)[-q], N\right) \stackrel{\cong}{\longrightarrow} \mathbb{H}^{q}\left(Y, \mathcal{H} \mathrm{om}_{\mathcal{O}_{Y}}\left(\gamma^{*} \mathbb{E}_{M}, \mathcal{N}\right)\right)$.

By Theorem 3.2 and the naturality of the maps in Proposition 2.16 the following diagram is commutative:

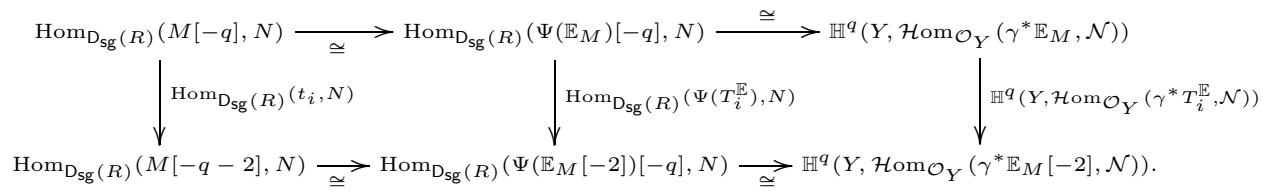

Now by Proposition 3.8 we have a natural map

$$
\mathbb{H}^{q}\left(Y, \mathcal{H} \operatorname{om}_{\mathcal{O}_{Y}}\left(\gamma^{*} \mathbb{E}_{M}, \mathcal{N}\right)\right) \rightarrow \Gamma\left(Y, \widehat{\mathcal{E x t}}_{\mathcal{O}_{Y}}^{q}\left(\operatorname{coker}\left(\mathbb{E}_{M}\right), \mathcal{N}\right)\right),
$$

noting that $\mathbb{E}_{\text {coker }\left(\mathbb{E}_{M}\right)} \cong \mathbb{E}_{M}$. This gives a commutative diagram

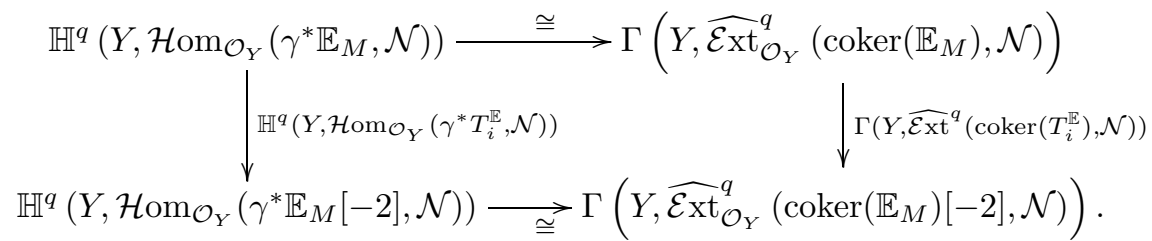

One checks that the following diagram commutes:

$$
\begin{aligned}
& \Gamma\left(Y, \widehat{\mathcal{E x t}}_{\mathcal{O}_{Y}}^{q}\left(\operatorname{coker}\left(\mathbb{E}_{M}\right), \mathcal{N}\right)\right) \longrightarrow \Gamma\left(Y, \widehat{\mathcal{E x t}}_{\mathcal{O}_{Y}}^{q}\left(\operatorname{coker}\left(\mathbb{E}_{M}\right), \mathcal{N}\right)\right) \\
& \downarrow \Gamma\left(Y, \widehat{\mathcal{E x t}}^{q}\left(\operatorname{coker}\left(T_{i}^{\mathbb{E}}\right), \mathcal{N}\right)\right) \quad \downarrow \Gamma\left(Y, T_{i}\right) \\
& \Gamma\left(Y, \widehat{\mathcal{E x t}}_{\mathcal{O}_{Y}}^{q}\left(\operatorname{coker}\left(\mathbb{E}_{M}\right)[-2], \mathcal{N}\right)\right) \cong \Gamma\left(Y, \widehat{\mathcal{E}}^{q}{ }_{\mathcal{O}_{Y}}\left(\operatorname{coker}\left(\mathbb{E}_{M}\right), \mathcal{N}\right)(1)\right)
\end{aligned}
$$

where the isomorphism

$$
\Gamma\left(Y, \widehat{\mathcal{E} x t}_{\mathcal{O}_{Y}}^{q}\left(\operatorname{coker}\left(\mathbb{E}_{M}\right)[-2], \mathcal{N}\right)\right) \stackrel{\cong}{\rightarrow} \Gamma\left(Y, \widehat{\mathcal{E} \times t}_{\mathcal{O}_{Y}}^{q}\left(\operatorname{coker}\left(\mathbb{E}_{M}\right), \mathcal{N}\right)(1)\right)
$$

is from Lemma 3.8 and $T_{i}$ is multiplication by $T_{i} \in \Gamma(Y, \mathcal{O}(1))$ on the coherent sheaf ${\widehat{\mathcal{E} \mathrm{xt}_{\mathcal{O}_{Y}}}}^{q}\left(\operatorname{coker}\left(\mathbb{E}_{M}\right), \mathcal{N}\right)$. 
Finally, we claim that there is a natural isomorphism $\mathcal{M} \cong \operatorname{coker}\left(\mathbb{E}_{M}\right)$ in $\mathrm{D}_{\mathrm{sg}}^{\mathrm{rel}}\left(Y \subseteq \mathbb{P}_{Q}^{\mathrm{c}-1}\right)$. Indeed, since $\Psi\left(\mathbb{E}_{M}\right):=\mathbf{R} \pi_{*} \beta^{b} \operatorname{coker}\left(\mathbb{E}_{M}\right) \cong M$ in $\mathrm{D}_{\mathrm{sg}}^{\mathrm{rel}}(Q \rightarrow R)$, we have that

$$
\mathbf{R} \beta_{*} \mathbf{L} \pi^{*} \mathbf{R} \pi_{*} \beta^{\mathrm{b}} \operatorname{coker}\left(\mathbb{E}_{M}\right) \cong \mathbf{R} \beta_{*} \mathbf{L} \pi^{*} M=\mathcal{M} \in \mathrm{D}_{\mathrm{sg}}^{\mathrm{rel}}\left(Y \hookrightarrow \mathbb{P}_{Q}^{\mathrm{c}-1}\right) .
$$

Since $\mathbf{R} \beta_{*} \mathbf{L} \pi^{*}$ induces an equivalence $\mathrm{D}_{\mathrm{sg}}^{\mathrm{rel}}(Q \rightarrow R) \rightarrow \mathrm{D}_{\mathrm{sg}}^{\mathrm{rel}}\left(Y \hookrightarrow \mathbb{P}_{Q}^{\mathrm{c}-1}\right)$ that is inverse to $\mathbf{R} \pi_{*} \beta^{\text {b }}$, the natural map $\mathbf{R} \beta_{*} \mathbf{L} \pi^{*} \mathbf{R} \pi_{*} \beta^{\text {b }} \operatorname{coker}\left(\mathbb{E}_{M}\right) \rightarrow \operatorname{coker}\left(\mathbb{E}_{M}\right)$ is an isomorphism in $\mathrm{D}_{\mathrm{sg}}^{\mathrm{rel}}\left(Y \hookrightarrow \mathbb{P}_{Q}^{\mathrm{c}-1}\right)$. Thus there exists a functorial isomorphism

$$
\mathcal{M}:=\mathbf{R} \beta_{*} \mathbf{L} \pi^{*} M \stackrel{\cong}{\longrightarrow} \operatorname{coker}\left(\mathbb{E}_{M}\right) .
$$

By the claim, there is a natural isomorphism $\widehat{\mathcal{E x t}}_{\mathcal{O}_{Y}}^{q}\left(\operatorname{coker}\left(\mathbb{E}_{M}\right), \mathcal{N}\right) \cong \widehat{\mathcal{E x t}}_{\mathcal{O}_{Y}}^{q}(\mathcal{M}, \mathcal{N})$.

Piecing together the commutative squares and the isomorphism $\mathcal{M} \cong$ coker $\mathbb{E}_{M}$ gives the commutative diagram in the statement of the corollary.

That the horizontal morphisms are isomorphisms for $q \gg 0$ follows from Proposition 3.8 .

We return again to the more general context that $X$ is a Noetherian scheme that is projective over an affine scheme, $\mathcal{L}$ is the corresponding very ample line bundle, $W$ is a regular global section of $\mathcal{L}$, and $\gamma: Z \hookrightarrow X$ is the zero subscheme of $W$. Recall that $\mathcal{E x t}_{\mathcal{O}_{Z}}^{q}(\mathcal{M}, \mathcal{N})$ denotes the quasi-coherent sheaf satisfying

$$
\mathcal{E x t}_{\mathcal{O}_{Z}}^{q}(\mathcal{M}, \mathcal{N})_{z} \cong \operatorname{Ext}_{\mathcal{O}_{Z, z}}^{q}\left(\mathcal{M}_{z}, \mathcal{N}_{z}\right)
$$

for all $z \in Z$.

Proposition 3.11. For each $q \in \mathbb{Z}$, there is a natural transformation

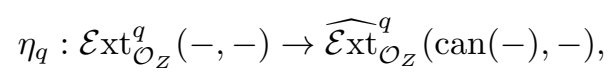

of functors from $\operatorname{RPerf}(Z \hookrightarrow X)^{\mathrm{op}} \times \mathrm{D}^{\mathrm{b}}(Z)$ to $\operatorname{coh} Z$, where

$$
\text { can : } \operatorname{RPerf}(Z \hookrightarrow X) \rightarrow \operatorname{RPerf}(Z \hookrightarrow X) / \operatorname{Perf} Z=\mathrm{D}_{\mathrm{sg}}^{\mathrm{rel}}(Z \hookrightarrow X)
$$

is the localization functor. For all $y \in Z$, the induced map on stalks

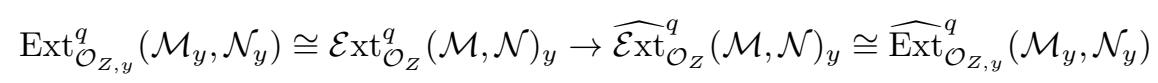

is the map (2.13). Moreover, for fixed $\mathcal{M} \in \operatorname{RPerf}(Z \hookrightarrow X)$ and $\mathcal{N} \in \mathrm{D}^{\mathrm{b}}(Z)$, there exists $q_{0} \geq 0$ such that $\eta_{q}$ is an isomorphism for all $q \geq q_{0}$.

Remark 3.12. Corollary 3.9 and Proposition 3.11 establish (1.2) and (1.3) from the introduction.

In the case of an affine scheme, say $Z=\operatorname{Spec} A$ and $\mathcal{M}=\widetilde{M}$ for an $A$-module $M$, one may compute $\widehat{\operatorname{Ext}}_{A}^{*}(M,-)$ using a complete resolution $T \rightarrow P \rightarrow M$ by Lemma B.6. In this case the map $T \rightarrow P$ induces the natural transformation $\operatorname{Ext}_{A}^{*}(M,-) \rightarrow \widehat{\operatorname{Ext}}_{A}^{*}(M,-)$.

In the more general case, $\gamma^{*} \mathbb{E}_{\mathcal{M}}$ represents, in some sense, the $T$ in a complete resolution of $\mathcal{M}$ in $\mathrm{D}_{\mathrm{sg}}^{\mathrm{rel}}(Z \hookrightarrow X)$. However there is not necessarily a locally free resolution $\mathcal{P}$ of $\mathcal{M}$ and a comparison map $\gamma^{*} \mathbb{E}_{\mathcal{M}} \rightarrow \mathcal{P}$. Thus to define the natural transformation in Proposition 3.11 requires more work than in the affine case. Our approach is to construct a global version of the Eisenbud operators. 
We begin by proving that given a complex $\mathcal{M}=\left(\cdots \rightarrow \mathcal{M}_{j+1} \rightarrow \mathcal{M}_{j} \rightarrow \cdots\right)$ of coherent sheaves on $Z$ that is bounded to the right (i.e., $\mathcal{M}_{j}=0$ for $j \ll 0$ ), there exists a sequence of maps of locally free coherent sheaves on $X$,

$$
\mathcal{E}=\cdots \rightarrow \mathcal{E}_{j+1} \stackrel{d_{j+1}}{\longrightarrow} \mathcal{E}_{j} \stackrel{d_{j}}{\longrightarrow} \mathcal{E}_{j-1} \rightarrow \cdots,
$$

and maps $g_{j}: \mathcal{E}_{j} \rightarrow \gamma_{*} \mathcal{M}_{j}$ of coherent sheaves on $X$, for all $j$, such that the following conditions hold: (1) $\mathcal{E}_{j}=0$ for $j \ll 0$, (2) the square

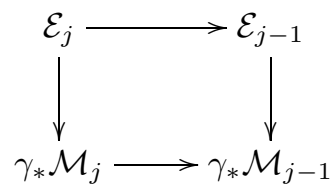

commutes for all $j,(3)$ the sequence $\gamma^{*}(\mathcal{E})$ is a complex, and $(4)$ the map $\gamma^{*}(\mathcal{E}) \rightarrow$ $\mathcal{M}$ of complexes given by the adjoints of the $g_{j}$ 's is a quasi-isomorphism. Note that $\mathcal{E}$ itself need not be complex.

It suffices to construct such sheaves and maps of sheaves so that (1) - (3) and the following condition hold: (4') the canonical map from $\gamma^{*} \mathcal{E}_{j}$ to the pullback of

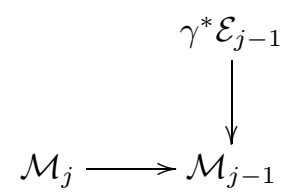

is surjective for all $j$. Indeed, an easy diagram chase shows that, so long as $\gamma^{*} \mathcal{E}$ is a complex, condition (4') implies that $\gamma^{*} \mathcal{E} \rightarrow \mathcal{M}$ is a quasi-isomorphism.

We give a recursive construction of $\mathcal{E}_{j}, d_{j}$ and $g_{j}$. Since $\mathcal{M}_{j}=0$ for $j \ll 0$, there is no problem starting the construction. Suppose $\mathcal{E}_{j}, d_{j}$, and $g_{j}$ have been constructed for all $j \leq n$ so that (3.13) commutes, the composition of $\gamma^{*} \mathcal{E}_{j} \rightarrow$ $\gamma^{*} \mathcal{E}_{j-1} \rightarrow \gamma^{*} \mathcal{E}_{j-2}$ is the zero map, and the map from $\gamma^{*} \mathcal{E}_{j}$ to the pullback of (3.14) is surjective, for all $j \leq n$. Observe also that these conditions ensure that $\gamma^{*} \mathcal{E}_{j} \rightarrow \mathcal{M}_{j}$ is surjective for all $j \leq n$, since $\mathcal{M}_{j}=0$ for $j \ll 0$.

Now define $\mathcal{F}_{n+1}$ to be the coherent sheaf fitting into the pullback square

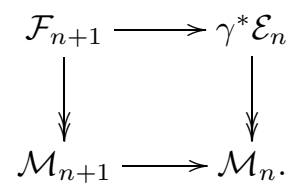

Then we can construct a locally free coherent sheaf $\mathcal{E}_{n+1}$ and maps so that

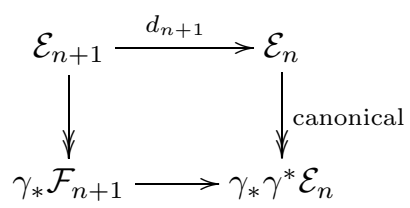

commutes and the left vertical map is surjective, by choosing $\mathcal{E}_{n+1}$ to be a locally free coherent sheaf mapping onto the pullback of the other three components of this square. Define $g_{n+1}$ as the composition $\mathcal{E}_{n+1} \rightarrow \gamma_{*} \mathcal{F}_{n+1} \rightarrow \gamma_{*} \mathcal{M}_{n+1}$. One may readily verify that the above conditions are now satisfied for all $j \leq n+1$, completing the recursive construction. 
Tensoring the exact sequence $0 \rightarrow \mathcal{L}^{-1} \rightarrow \mathcal{O}_{X} \rightarrow \gamma_{*} \mathcal{O}_{Z} \rightarrow 0$ with any locally free coherent sheaf $\mathcal{G}$ on $X$ gives an exact sequence

$$
0 \rightarrow \mathcal{G} \otimes \mathcal{L}^{-1} \rightarrow \mathcal{G} \rightarrow \gamma_{*} \gamma^{*} \mathcal{G} \rightarrow 0
$$

It follows that for every $j$, the composite map

$$
\mathcal{E}_{j} \rightarrow \mathcal{E}_{j-2}
$$

factors through a uniquely determined map

$$
\tilde{t}_{j}: \mathcal{E}_{j} \rightarrow \mathcal{E}_{j-2} \otimes \mathcal{L}^{-1}
$$

Let $t_{j}=\gamma^{*}\left(\tilde{t}_{j}\right)$. By localizing and using the results of [15, Section 1], we see that the collection $t_{j}$ determines a map of chain complexes

$$
t: \gamma^{*}(\mathcal{E}) \rightarrow \gamma^{*}\left(\mathcal{E} \otimes \mathcal{L}^{-1}\right)[-2] .
$$

Lemma 3.15. For a complex $\mathcal{M}$ on $Z$ with bounded coherent cohomology, choose $\mathcal{E}$ and define $t: \gamma^{*}(\mathcal{E}) \rightarrow \gamma^{*}\left(\mathcal{E} \otimes \mathcal{L}^{-1}\right)[-2]$ as above. Then $t$ induces a natural transformation of functors from $\operatorname{coh} Z$ to $\operatorname{coh} Z$,

$$
\chi_{(X, \mathcal{L}, W)}^{q}: \mathcal{E}_{\mathrm{xt}_{\mathcal{O}_{Z}}^{q}}^{q}(\mathcal{M},-)(1) \rightarrow \mathcal{E}_{\mathrm{xt}_{\mathcal{O}_{Z}}^{q+2}(\mathcal{M},-)} .
$$

Moreover, this map is natural in $\mathcal{M}$ and it is the unique map such that, for all $y \in Z$, the induced map on stalks at $y$,

$$
\operatorname{Ext}_{\mathcal{O}_{z, y}}^{q}\left(\mathcal{M}_{y}, \mathcal{N}_{y}\right) \otimes\left(I / I^{2}\right)^{*} \rightarrow \operatorname{Ext}_{\mathcal{O}_{z_{y}}}^{q+2}(\mathcal{M}, \mathcal{N}),
$$

is the Eisenbud operator arising from the surjection $\mathcal{O}_{X, y} \rightarrow \mathcal{O}_{Z, y}$ with kernel I. In particular, it is independent of the choice of $\mathcal{E}$.

Remark 3.16. We have the exact sequence $0 \rightarrow \mathcal{L}^{-1} \rightarrow \mathcal{O}_{X} \rightarrow \gamma_{*} \mathcal{O}_{Z} \rightarrow 0$, and hence the stalk of $\mathcal{L}^{-1}$ at $y$ is identified with $I$ and the stalk of $\gamma^{*} \mathcal{L}^{-1}$ is identified with $I / I^{2}$.

Now, $I / I^{2}$ is a free $\mathcal{O}_{Z, y}$-module of rank 1 , and picking a generator $f$ of the principal ideal $I$ determines a basis of $I / I^{2}$ and hence yields the more customary form of the Eisenbud operator for a hypersurface:

$$
\operatorname{Ext}_{\mathcal{O}_{z, y}}^{q}\left(\mathcal{M}_{y}, \mathcal{N}_{y}\right) \rightarrow \operatorname{Ext}_{\mathcal{O}_{z_{y}}+2}^{q+2}\left(\mathcal{M}_{y}, \mathcal{N}_{y}\right) \text {. }
$$

But, this map depends on the choice of $f$ - any other generator of $I$ has the form $u f$ for a unit $u$ of $\mathcal{O}_{X, x}$, and the operator obtained from the choice of $u f$ is given by $\bar{u}$ times the operator arising from $f$.

Proof. Observe that the uniqueness assertion, once established, will give that the maps $\chi_{(X, \mathcal{L}, W)}^{q}$ do not depend on the choice of $\mathcal{E}$ and hence they determine a natural transformation. We use the locally free resolution $\gamma^{*} \mathcal{E}$ of $\mathcal{M}$ to compute $\mathcal{E}_{\mathrm{xt}_{\mathcal{O}_{Z}}^{*}}(\mathcal{M}, \mathcal{N})$. The chain map $t: \gamma^{*}(\mathcal{E}) \rightarrow \gamma^{*}\left(\mathcal{E} \otimes \mathcal{L}^{-1}\right)$ induces

$$
\chi_{(X, \mathcal{L}, W)}^{q}: \mathcal{E}_{\mathrm{xt}_{\mathcal{O}_{Z}}^{q}}^{q}(\mathcal{M}, \mathcal{N})(1) \rightarrow \mathcal{E}_{\mathrm{xt}_{\mathrm{O}_{Z}}^{q+2}(\mathcal{M}, \mathcal{N})}
$$

for every $q \geq 0$. It follows from the construction that, locally, this agrees with the map constructed by Eisenbud. The naturality and uniqueness assertions may also be verified locally and thus follow from [15, Section 1]. 
Proof of Proposition 3.11, Recall that by definition

$$
{\widehat{\mathcal{E} \mathrm{xt}_{\mathcal{O}_{Z}}}}_{r}^{r}(\mathcal{M}, \mathcal{N})=\mathcal{H}^{r} \mathcal{H o m}\left(\gamma^{*} \mathbb{E}_{\mathcal{M}}, \mathcal{N}\right)
$$

where $\mathbb{E}_{\mathcal{M}}$ denotes the image of $\mathcal{M}$ under the fixed inverse of the functor coker. For $r \geq 1$, there is a natural isomorphism $\mathcal{H}^{r} \mathcal{H o m}\left(\gamma^{*} \mathbb{E}_{\mathcal{M}}, \mathcal{N}\right) \cong \mathcal{E}_{\mathrm{xt}^{r}}^{r}\left(\operatorname{coker} \mathbb{E}_{\mathcal{M}}, \mathcal{N}\right)$ since $\gamma^{*} \mathbb{E}_{\mathcal{M}}$ is a locally free resolution of coker $\mathbb{E}_{\mathcal{M}}$ by [13, 5.2.1]. There is an

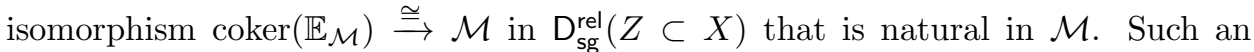
isomorphism is an equivalence class of diagrams

$$
\mathcal{M} \leftarrow \mathcal{G} \rightarrow \operatorname{coker}\left(\mathbb{E}_{\mathcal{M}}\right)
$$

in $\operatorname{RPerf}(Z \hookrightarrow X)$ such that the cones of both morphisms lie in $\operatorname{Perf}(Z)$.

For $\mathcal{P} \in \operatorname{Perf}(Z)$ and any $\mathcal{N}$, we have $\mathcal{E}_{\mathrm{xt}_{\mathcal{O}_{Z}}^{q}}^{q}(\mathcal{P}, \mathcal{N})=0$ for $q \gg 0$. It follows that, given $\mathcal{N}$, there is an integer $r_{0}$ such that both arrows in

$$
\mathcal{E}_{\mathrm{xt}^{r}}^{r} \mathrm{O}_{Z}(\mathcal{M}, \mathcal{N}) \cong \mathcal{E}_{\mathrm{xt}_{\mathcal{O}_{Z}}^{r}}^{r}(\mathcal{G}, \mathcal{N}) \cong \mathcal{E}_{\mathrm{xt}_{\mathcal{O}_{Z}}^{r}}\left(\operatorname{coker}\left(\mathbb{E}_{\mathcal{M}}\right), \mathcal{N}\right)
$$

are isomorphisms for $r \geq r_{0}$. This determines a family of isomorphisms

$$
\eta_{r}: \mathcal{E}_{\mathrm{xt}_{\mathcal{O}_{Z}}^{r}}^{r}(\mathcal{M}, \mathcal{N}) \cong{\widehat{\mathcal{E} \mathrm{xt}_{\mathcal{O}_{Z}}}}^{r}(\mathcal{M}, \mathcal{N}), r \geq r_{0} .
$$

The induced map on the stalk at any point $y$ is given by the usual map from ordinary to stable Ext-modules by Lemma B.6 and Proposition 3.8. This proves, in particular, that for $r \geq r_{0}, \eta_{r}$ is natural in both arguments and that it is independent of choices.

Finally, to complete the proof of the proposition, we extend the definition of $\eta_{r}$ to all $r$ by using Lemma 3.15, defining $\eta_{r}$ to be the composition of

$$
\begin{aligned}
& \mathcal{E}_{\mathrm{xt}_{\mathcal{O}_{Z}}^{r}}(\mathcal{M}, \mathcal{N}) \stackrel{\chi^{\circ N}}{\longrightarrow} \mathcal{E}_{\mathrm{xt}^{r+2 N}}^{r+\mathcal{O}}(\mathcal{M}, \mathcal{N})(-N) \\
& \stackrel{\eta_{r+2 N}}{\longrightarrow}{\widehat{\mathcal{E} \mathrm{xt}_{\mathcal{O}_{Z}}}}_{r+2 N}^{r}(\mathcal{M}, \mathcal{N})(-N) \stackrel{\cong}{\rightrightarrows} \widehat{\mathcal{E x t}}_{\mathcal{O}_{Z}}^{r}(\mathcal{M}, \mathcal{N})
\end{aligned}
$$

for $N \gg 0$, where the isomorphism on the right is from Proposition 3.8. To see that the definition of $\eta_{r}$ is independent of the choice of $N \gg 0$ and that the resulting map is natural, it suffices to localize at an arbitrary point. Locally, these properties are seen by combining Lemma $\left[3.15\right.$, the fact that $\widehat{\mathcal{E x t}}_{\mathcal{O}_{Z}}^{r}(\mathcal{M}, \mathcal{N}) \stackrel{\cong}{\rightrightarrows}$ ${\widehat{\mathcal{E} \mathrm{xt}_{\mathcal{O}_{Z}}}}^{r+2 N}(\mathcal{M}, \mathcal{N})(-N)$ is, locally, given by the Eisenbud operator, and the fact that the canonical map from Ext to $\widehat{E x t}$ commutes with the Eisenbud operator. The latter property is seen to hold by using a complete resolution to compute the stable Ext modules.

3.2. Some additional corollaries. Again we return to the context and notation of 92.3 . For complexes of $R$-modules $M$ and $N$ with bounded and finitely generated cohomology, we define the graded $R\left[T_{1}, \ldots, T_{c}\right]$ modules:

$$
\begin{aligned}
\operatorname{Ext}_{R}^{e v}(M, N) & =\bigoplus_{n \geq 0} \operatorname{Ext}_{R}^{2 n}(M, N) \\
\operatorname{Ext}_{R}^{\text {odd }}(M, N) & =\bigoplus_{n \geq 0} \operatorname{Ext}_{R}^{2 n+1}(M, N),
\end{aligned}
$$

where $\operatorname{Ext}_{R}^{2 n}(M, N)$ and $\operatorname{Ext}_{R}^{2 n+1}(M, N)$ lie in degree $n$ and the $T_{i}$ 's act as $\operatorname{Ext}_{R}^{n}\left(t_{i}, N\right)$ for $t_{i}$ the $i$-th Eisenbud operator on $M$. 
When $M$ has finite projective dimension over $Q$, Gulliksen proved in [17 that $\operatorname{Ext}_{R}^{e v}(M, N)$ and $\operatorname{Ext}_{R}^{\text {odd }}(M, N)$ are finitely generated graded modules over the ring $R\left[T_{1}, \ldots, T_{c}\right]$. They therefore determine coherent sheaves on $\mathbb{P}_{R}^{\mathrm{c}-1}$, which we write as $\left.\operatorname{Ext}_{R}^{\text {ev }(M}, N\right)$ and $\left.\operatorname{Ext}_{R}^{\text {odd }(M}, N\right)$. Note that while Gulliksen did not use Eisenbud operators, it was shown in [7] that the action used by Gulliksen coincides, up to a sign, with the Eisenbud operators, and moreover Gulliksen's result, which was originally stated only for modules, was extended to complexes of $R$-modules with bounded and finitely generated cohomology.

Corollary 3.18. Let $M$ and $N$ be complexes of $R$-modules with bounded and finitely generated cohomology and assume that $M$ is perfect over $Q$. Set $\mathcal{M}=\beta_{*} \pi^{*} M$ and $\mathcal{N}=\beta_{*} \pi^{*} N$, where $\beta: \mathbb{P}_{R}^{c-1} \hookrightarrow Y$ is the canonical inclusion and $\pi: \mathbb{P}_{R}^{c-1} \rightarrow \operatorname{Spec} R$ is the canonical proper map. For $q \gg 0$, there are isomorphisms of coherent sheaves on $Y$ :

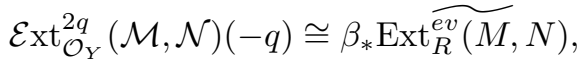

$$
\begin{aligned}
& \left.\mathcal{E}_{\operatorname{xt}^{2}}^{2 q+1}(\mathcal{M}, \mathcal{N})(-q) \cong \beta_{*} \operatorname{Ext}_{R}^{\widetilde{o d d}(M}, N\right) \text {. }
\end{aligned}
$$

Proof. For $n \gg 0$, we have natural isomorphisms $\operatorname{Ext}_{R}^{n}(M, N) \cong \widehat{\operatorname{Ext}}_{R}^{n}(M, N)$ that commute with the Eisenbud operators. It thus follows from Corollary 3.9 and Proposition 3.11 that, for $q \gg 0$, we have isomorphisms

$$
\bigoplus_{n \geq q} \operatorname{Ext}_{R}^{2 n}(M, N) \cong \bigoplus_{n \geq q} \mathrm{H}^{0}\left(Y, \mathcal{E x t}_{\mathcal{O}_{Y}}^{2 n}(\mathcal{M}, \mathcal{N})\right) \cong \bigoplus_{n \geq q} \mathrm{H}^{0}\left(Y, \mathcal{E x t}_{\mathcal{O}_{Y}}^{2 q}(\mathcal{M}, \mathcal{N})(n-q)\right)
$$

of graded $R\left[T_{1}, \ldots, T_{c}\right]$-modules. The coherent sheaf on $Y$ associated to the first of these is $\beta_{*} \operatorname{Ext}_{R} \widehat{e v}(M, N)$, and the coherent sheaf associated to the last of these is $\mathcal{E x t}_{\mathcal{O}_{Y}}^{2 q}(\mathcal{M}, \mathcal{N})(-q)$.

The proof for the odd degree case is identical.

Remark 3.19. The results above recover Gulliksen's theorem on the finiteness of $\operatorname{Ext}_{R}^{*}(M, N)$ over $R\left[T_{1}, \ldots, T_{c}\right]$. Indeed, since $\mathcal{E x t}_{\mathcal{O}_{Y}}^{q}(\mathcal{M}, \mathcal{N})$ is coherent, so is $\gamma_{*} \mathcal{E}_{\mathrm{xt}^{q}}^{q}(\mathcal{M}, \mathcal{N})$, where $\gamma: Y \hookrightarrow \mathbb{P}_{Q}^{\mathrm{c}-1}$ is the canonical inclusion. Thus the $Q\left[T_{1}, \ldots, T_{c}\right]$-module

$$
\bigoplus_{n \geq 0} \mathrm{H}^{0}\left(\mathbb{P}_{Q}^{\mathrm{c}-1}, \gamma_{*} \mathcal{E}_{\mathrm{xt}_{\mathcal{O}_{Y}}^{d}}(\mathcal{M}, \mathcal{N})(n)\right)
$$

is finitely generated. Hence so is $\left(\operatorname{Ext}_{R}^{e v}(M, N)\right)_{\geq q}$ for $q \gg 0$, by Corollary 3.9 and Proposition 3.11. Adding on the finitely generated $R$-module $\left(\operatorname{Ext}_{R}^{e v}(M, N)\right)_{<q}$, we see that $\operatorname{Ext}_{R}^{e v}(M, N)$ is finitely generated over $Q\left[T_{1}, \ldots, T_{c}\right]$. Finally, since $\operatorname{Ext}_{R}^{e v}(M, N)$ is annihilated by $f_{1}, \ldots, f_{c}$, it is finitely generated over $R\left[T_{1}, \ldots, T_{c}\right]$.

Corollary 3.20. Let $M$ and $N$ be complexes of $R$-modules with bounded and finitely generated cohomology and assume that $M$ is perfect over $Q$. Let $\mathcal{M}=\beta_{*} \pi^{*} M$ and $\mathcal{N}=\beta_{*} \pi^{*} N$. For all $n \in \mathbb{Z}$, the support of the $\mathcal{O}_{Y}$-module ${\widehat{\mathcal{E} t_{t}}}_{\mathcal{O}_{Y}}^{n}(\mathcal{M}, \mathcal{N})$ is contained in $\mathbb{P}_{R}^{c-1}$, and we have equalities

$$
\begin{aligned}
& \operatorname{supp} \widehat{\mathcal{E x t}}_{\mathcal{O}_{Y}}^{0}(\mathcal{M}, \mathcal{N})=\operatorname{supp} \operatorname{Ext}_{R}^{\widehat{e v}(M, N)}, \\
& \operatorname{supp}{\widehat{\mathcal{E} \operatorname{xt}_{\mathcal{O}_{Y}}}}^{T}(\mathcal{M}, \mathcal{N})=\operatorname{supp} \operatorname{Ext}_{R}^{\text {odd }(M, N)},
\end{aligned}
$$

of closed subsets of $\mathbb{P}_{R}^{c-1}$. 
Proof. For $n \gg 0$ we have that ${\widehat{\mathcal{E} \mathrm{xt}_{\mathcal{O}_{Y}}}}^{n}(\mathcal{M}, \mathcal{N}) \cong \mathcal{E}_{\mathrm{xt}_{\mathcal{O}_{Y}}^{n}}(\mathcal{M}, \mathcal{N})$ by Proposition 3.11, For $q \gg 0$, by Corollary 3.18 we have that $\mathcal{E x t}_{\mathcal{O}_{Y}}^{2 q}(\mathcal{M}, \mathcal{N})(-q) \cong \beta_{*} \operatorname{Ext}_{R}^{\widetilde{e v}(M, N)}$ and $\left.\mathcal{E}_{\mathrm{xt}^{2}}^{2 q+1}(\mathcal{M}, \mathcal{N})(-q) \cong \beta_{*} \operatorname{Ext}_{R}^{\text {odd }(M}, N\right)$. Finally, by Proposition 3.8 , ${\widehat{\mathcal{E} \mathrm{xt}^{\mathcal{O}_{Y}}}}^{2 q}(\mathcal{M}, \mathcal{N})(-q) \cong{\widehat{\mathcal{E} \mathrm{Xt}_{\mathcal{O}_{Y}}}}^{0}(\mathcal{M}, \mathcal{N})$, and similarly for the odd case.

We close with a definition.

Definition 3.21. Let $M$ and $N$ be complexes of $R$-modules with bounded and finitely generated cohomology and assume that $M$ is perfect over $Q$. The stable support set of $(M, N)$ is the closed subset $V_{Q}^{\mathbf{f}}(M, N)$ of $\mathbb{P}_{R}^{\mathrm{c}-1}$ defined as

$$
\left.\left.V_{Q}^{\mathbf{f}}(M, N):=\operatorname{supp} \operatorname{Ext}_{R}^{e_{R}^{e v}(M}, N\right) \cup \operatorname{supp} \operatorname{Ext}_{R}^{\text {odd }(M}, N\right) .
$$

The stable support set of $M$ is defined to be $V_{Q}^{\mathbf{f}}(M):=V_{Q}^{\mathbf{f}}(M, M)$.

In Section 8 we study this support further and clarify its relation to the notions of support for $R$-modules defined previously in [4, $, 8,32$.

Remark 3.22. By Corollary 3.20, there is an equality

$$
V_{Q}^{\mathbf{f}}(M, N)=\operatorname{supp}{\widehat{\mathcal{E} \mathrm{xt}_{\mathcal{O}_{Y}}}}^{0}(\mathcal{M}, \mathcal{N}) \cup \operatorname{supp}{\widehat{\mathcal{E} \mathrm{xt}_{\mathcal{O}_{Y}}}}^{1}(\mathcal{M}, \mathcal{N}) .
$$

If $N$ is also perfect over $Q$, then there is an equality

$$
V_{Q}^{\mathbf{f}}(M, N)=\operatorname{supp} \mathcal{H}^{2 i} \mathcal{H} \mathrm{om}_{\mathrm{MF}}\left(\mathbb{E}_{M}, \mathbb{E}_{N}\right) \cup \operatorname{supp} \mathcal{H}^{2 i+1} \mathcal{H} \mathrm{om}_{\mathrm{MF}}\left(\mathbb{E}_{M}, \mathbb{E}_{N}\right)
$$

for any $i \in \mathbb{Z}$. This follows from Remark 3.5 and the definition of $\mathcal{H} \mathrm{om}_{\mathrm{MF}}\left(\mathbb{E}_{M}, \mathbb{E}_{N}\right)$ as a twisted periodic complex which shows that

$$
\mathcal{H}^{i+2} \mathcal{H} \mathrm{om}_{\mathrm{MF}}\left(\mathbb{E}_{M}, \mathbb{E}_{N}\right) \cong\left(\mathcal{H}^{i} \mathcal{H} \mathrm{om}_{\mathrm{MF}}\left(\mathbb{E}_{M}, \mathbb{E}_{N}\right)\right)(1)
$$

for all $i \in \mathbb{Z}$.

Remark 3.23. In fact, there is a containment

$$
\operatorname{supp} \widehat{\mathcal{E x t}}_{\mathcal{O}_{Y}}^{0}(\mathcal{M}, \mathcal{M}) \supseteq \operatorname{supp} \widehat{\mathcal{E x t}}_{\mathcal{O}_{Y}}^{1}(\mathcal{M}, \mathcal{M}) \text {. }
$$

Indeed, for a local hypersurface ring $T$, and a finite $T$-module $M$, it follows from [4, 4.2] that $M$ has finite projective dimension if and only if $\widehat{\operatorname{Ext}}_{T}^{0}(M, M)=0$, which implies that $\widehat{\operatorname{Ext}}_{T}^{1}(M, M)=0$.

\section{Proof of Theorem 3.2}

In this section we prove Theorem 3.2 . The first step is to associate to a matrix factorization $\mathbb{E}$ an explicit complex of finitely generated projective $R$-modules that represents $\Psi(\mathbb{E})$ in $D_{\mathrm{sg}}(R)$, where $\Psi$ is the equivalence of Corollary 2.11. We remain in the context and under the assumptions of $\$ 2.3$

For any scheme $X$, we write $\mathcal{C}(X)$ for the category of arbitrary complexes of quasi-coherent sheaves and $\mathcal{C}^{b}(X)$ for the full subcategory consisting of complexes with bounded and coherent cohomology. If $X=\operatorname{Spec} R$, we write $\mathcal{C}(R)$ and $\mathcal{C}^{b}(R)$.

Definition 4.1. Set $\delta^{\sharp}$ to be the additive functor from $M F\left(\mathbb{P}_{Q}^{\mathrm{c}-1}, \mathcal{O}(1), W\right)$ to $\mathcal{C}^{b}\left(\mathbb{P}_{R}^{\mathrm{c}-1}\right)$ that sends an object $\mathbb{E}=\left(\mathcal{E}_{1} \rightarrow \mathcal{E}_{0} \rightarrow \mathcal{E}_{1}(1)\right)$ to

$$
\delta^{\sharp} \mathbb{E}:=\left(\cdots \rightarrow \delta^{*} \mathcal{E}_{0}(-2) \rightarrow \delta^{*} \mathcal{E}_{1}(-1) \rightarrow \delta^{*} \mathcal{E}_{0}(-1) \rightarrow 0\right),
$$


where $\delta^{*} \mathcal{E}_{0}(-1)$ is located in cohomological degree $c-1$. The action on morphisms is the obvious one. Recall that $\delta: \mathbb{P}_{R}^{\mathrm{c}-1} \hookrightarrow \mathbb{P}_{Q}^{\mathrm{c}-1}$ is the canonical inclusion.

Remark 4.2. The complex $\delta^{\sharp} \mathbb{E}$ is exact everywhere except on the right (i.e., except in degree $c-1)$. Indeed by [13, 5.2.1], $\gamma^{*} \mathbb{E}$ is an exact complex of locally free sheaves, where $\gamma: Y \hookrightarrow \mathbb{P}_{Q}^{\mathrm{c}-1}$ is the canonical inclusion and $\gamma^{*} \mathbb{E}$ is defined in (2.15). The sheaf $\mathcal{M}=\mathcal{H}^{0}\left(\gamma^{*} \mathbb{E} \leq 0\right)=$ coker $\partial_{\gamma^{*} \mathbb{E}}^{0}$ has an obvious right resolution by locally free sheaves. Thus since $\beta$ has finite flat dimension, where $\beta: \mathbb{P}_{R}^{c-1} \hookrightarrow Y$ is the canonical inclusion, $\mathbf{L} \beta^{*} \mathcal{M} \cong \beta^{*} \mathcal{M}$, i.e. $\mathbf{L} \beta^{*} \mathcal{M}$ has cohomology only in a single degree. Since $\gamma^{*} \mathbb{E} \leq 0$ is a locally free resolution of $\mathcal{M}$, this translates to the fact that $\beta^{*} \gamma^{*} \mathbb{E} \leq 0 \cong \delta^{*} \mathbb{E} \leq 0$ only has cohomology in a single degree. The claim follows immediately.

Recall that $\beta: \mathbb{P}_{R}^{\mathrm{c}-1} \hookrightarrow Y$ is the canonical inclusion, and that $\beta^{\mathrm{b}}$ is the right adjoint to $\mathbf{R} \beta_{*}$. By [18, III.1.5, III.7.3], the functor $\beta^{\text {b }}$ sends a complex $\mathcal{F}$ to $\mathbf{L} \beta^{*}(\mathcal{F})(-1)[-c+1]$. By [13, 5.2.1]

$$
\cdots \rightarrow \gamma^{*} \mathcal{E}_{1}(-1) \rightarrow \gamma^{*} \mathcal{E}_{0}(-1) \rightarrow 0
$$

is a locally free resolution of $\operatorname{coker}(\mathbb{E})(-1)$. Thus there is a functorial isomorphism in $\mathrm{D}^{\mathrm{b}}\left(\mathbb{P}_{R}^{\mathrm{c}-1}\right)$ :

$$
\mathbf{L} \beta^{*}(\operatorname{coker}(\mathbb{E})(-1))[-c+1] \cong \delta^{\sharp} \mathbb{E},
$$

since $\gamma \circ \beta=\delta$. We thus have a functorial isomorphism

$$
\delta^{\sharp} \mathbb{E} \cong \beta^{b} \text { coker } \mathbb{E}
$$

in the category $\mathrm{D}^{\mathrm{b}}\left(\mathbb{P}_{R}^{\mathrm{c}-1}\right)$.

Let $\mathcal{U}=\left(U_{1}, \ldots, U_{c}\right)$ denote the standard affine cover of $\mathbb{P}_{R}^{\mathrm{c}-1}$ and let $\mathcal{P}$ be a complex of quasi-coherent sheaves on $\mathbb{P}_{R}^{\mathrm{c}-1}$. As in $\operatorname{Section} 2, \Gamma(\mathcal{U}, \mathcal{P})$ is the total complex associated to the bicomplex given by applying the Cech construction with respect to the cover $\mathcal{U}$ degree-wise to $\mathcal{P}$. We view this as a functor $\Gamma(\mathcal{U},-)$ : $\mathcal{C}^{b}\left(\mathbb{P}_{R}^{c-1}\right) \rightarrow \mathcal{C}^{b}(R)$. There is a natural isomorphism

$$
\Gamma(\mathcal{U}, \mathcal{P}) \stackrel{\cong}{\longrightarrow} \pi_{*} \mathcal{P} \in \mathrm{D}^{\mathrm{b}}(R) .
$$

Combining (4.3) and (4.4) we see that for $\mathbb{E}$ a matrix factorization, we have a natural isomorphism:

$$
\mathbf{R} \pi_{*} \beta^{\mathrm{b}}(\operatorname{coker}(\mathbb{E})) \cong \Gamma\left(\mathcal{U}, \delta^{\sharp} \mathbb{E}\right) .
$$

Since $\mathbf{R} \pi_{*} \beta^{b}(\operatorname{coker}(-))$ induces the equivalence $\Psi:[M F] \rightarrow \mathrm{D}_{\mathrm{sg}}^{\text {rel }}(Q \rightarrow R)$ of Corollary 2.11, we have proven the following:

Proposition 4.5. The functor $\Gamma\left(\mathcal{U}, \delta^{\sharp}-\right)$ induces a functor $\left[M F\left(\mathbb{P}_{Q}^{c-1}, \mathcal{O}(1), W\right)\right] \rightarrow$ $\mathrm{D}_{\mathrm{sg}}^{\mathrm{rel}}(Q \rightarrow R)$, which we also write as $\Gamma\left(\mathcal{U}, \delta^{\sharp}-\right)$, and there is an isomorphism of functors

$$
\Psi(-) \stackrel{\cong}{\longrightarrow} \Gamma\left(\mathcal{U}, \delta^{\sharp}-\right):\left[M F\left(\mathbb{P}_{Q}^{c-1}, \mathcal{O}(1), W\right)\right] \rightarrow \mathrm{D}_{\mathrm{sg}}^{\mathrm{rel}}(Q \rightarrow R),
$$

where $\Psi$ is the equivalence of Corollary 2.11, In particular, $\Gamma\left(\mathcal{U}, \delta^{\sharp}-\right)$ is a triangulated functor.

As the sheaves in the complex $\Gamma\left(\mathcal{U}, \delta^{\sharp} \mathbb{E}\right)$ are not coherent, we look for a complex of coherent sheaves that represents it in $\mathrm{D}_{\mathrm{sg}}^{\mathrm{rel}}(Q \rightarrow R)$. 
Definition 4.6. Define $F: \mathcal{C}^{b}\left(\mathbb{P}_{R}^{c-1}\right) \rightarrow \mathcal{C}(R)$ to be the functor given by applying

$$
\mathrm{H}^{c-1}\left(\mathbb{P}_{R}^{\mathrm{c}-1},-\right)[-c+1]
$$

degree-wise. In particular,

$$
\begin{aligned}
F\left(\delta^{\sharp} \mathbb{E}\right):=(\cdots & \rightarrow \mathrm{H}^{c-1}\left(\mathbb{P}_{R}^{\mathrm{c}-1}, \delta^{*} \mathcal{E}_{0}(-2)\right) \rightarrow \mathrm{H}^{c-1}\left(\mathbb{P}_{R}^{\mathrm{c}-1}, \delta^{*} \mathcal{E}_{1}(-1)\right) \\
& \left.\rightarrow \mathrm{H}^{c-1}\left(\mathbb{P}_{R}^{\mathrm{c}-1}, \delta^{*} \mathcal{E}_{0}(-1)\right) \rightarrow 0\right),
\end{aligned}
$$

with $\mathrm{H}^{c-1}\left(\mathbb{P}_{R}^{c-1}, \mathcal{E}_{0}(-1)\right)$ located in cohomological degree $2 c-2$.

If $\mathcal{E}$ is any coherent sheaf on $\mathbb{P}_{R}^{\mathrm{c}-1}$, there is a natural map of complexes

$$
\Gamma(\mathcal{U}, \mathcal{E}) \rightarrow \mathrm{H}^{c-1}(\Gamma(\mathcal{U}, \mathcal{E}))[-c+1]
$$

since $(\Gamma(\mathcal{U}, \mathcal{E}))^{i}=0$ for $i>c-1$. Since $\mathrm{H}^{i}(\Gamma(\mathcal{U}, \mathcal{E})) \cong \mathrm{H}^{i}\left(\mathbb{P}_{R}^{\mathrm{c}-1}, \mathcal{E}\right)$, the map (4.7) is a quasi-isomorphism if and only if $\mathrm{H}^{i}\left(\mathbb{P}_{R}^{\mathrm{c}-1}, \mathcal{E}\right)=0$ for all $i \neq c-1$. Applying (4.7) degree-wise to a chain complex $\mathcal{P}$ yields a map of bicomplexes, and taking the total complex gives a map $\eta_{\mathcal{P}}: \Gamma(\mathcal{U}, \mathcal{P}) \rightarrow F(\mathcal{P})$. It is natural in $\mathcal{P}$ and so gives a natural transformation

$$
\eta: \Gamma(\mathcal{U},-) \rightarrow F(-) .
$$

From this construction we see that if $\mathrm{H}^{i}\left(\mathbb{P}_{R}^{c-1}, \mathcal{P}^{j}\right)=0$ for all $i \neq c-1$ and all $j$, then $\eta_{\mathcal{P}}$ is a quasi-isomorphism. Also note that for all $\mathcal{P}$ there is a commutative diagram

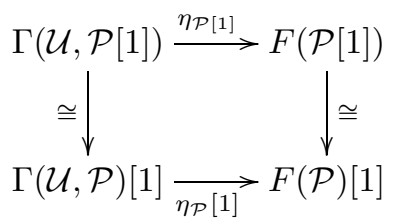

where [1] is the shift functor on the respective categories of complexes.

For a complex $\mathcal{P}$, recall that $\mathcal{P} \leq m$ is the brutal truncation in cohomological degree $m$. There are natural maps $\mathcal{P} \rightarrow \mathcal{P} \leq m$ and $\mathcal{P} \leq m \rightarrow \mathcal{P} \leq n$ for $m \geq n$.

Definition 4.10. An integer $m$ is sufficiently small for an object $\mathbb{E}=\left(\mathcal{E}_{1} \rightarrow \mathcal{E}_{0} \rightarrow\right.$ $\left.\mathcal{E}_{1}(1)\right)$ of $M F\left(\mathbb{P}_{Q}^{\mathrm{c}-1}, \mathcal{O}(1), W\right)$ if every coherent sheaf $\mathcal{E}$ appearing in the complex $\delta^{\sharp}(\mathbb{E}) \leq m=\left(\cdots \rightarrow \delta^{*} \mathcal{E}_{1}(-1) \rightarrow \delta^{*} \mathcal{E}_{0}(-1) \rightarrow 0\right) \leq m$ satisfies

(1) $\mathrm{H}^{i}\left(\mathbb{P}_{R}^{\mathrm{c}-1}, \mathcal{E}\right)=0$ for all $i \neq c-1$ and

(2) $\mathrm{H}^{c-1}\left(\mathbb{P}_{R}^{\mathrm{c}-1}, \mathcal{E}\right)$ is a projective $R$-module.

The name of this condition is justified by:

Lemma 4.11. Let $\mathbb{E}$ be an object of $M F\left(\mathbb{P}_{Q}^{c-1}, \mathcal{O}(1), W\right)$. There is an integer $m_{0}$, which depends on $\mathbb{E}$, such that all $m \leq m_{0}$ are sufficiently small for $\mathbb{E}$. Moreover, if $m$ is sufficiently small for $\mathbb{E}$, then $F\left(\left(\delta^{\sharp} \mathbb{E}\right) \leq m\right)$ is a right bounded complex of finitely generated projective $R$-modules and the map

$$
\eta: \Gamma\left(\mathcal{U},\left(\delta^{\sharp} \mathbb{E}\right) \leq m\right) \stackrel{\sim}{\longrightarrow} F\left(\left(\delta^{\sharp} \mathbb{E}\right) \leq m\right)
$$

is a quasi-isomorphism. 
Proof. Consider the complex $\delta^{\sharp} \mathbb{E}$. By construction, if for some $i$ the sheaf $\left(\delta^{\sharp} \mathbb{E}\right)^{i}$ is non-zero, then

$$
\left(\delta^{\sharp} \mathbb{E}\right)^{i-2} \cong\left(\delta^{\sharp} \mathbb{E}\right)^{i}(-1) .
$$

Let $(-)^{\vee}$ denote $\mathcal{H} \mathrm{om}_{\mathbb{P}_{R}^{\mathrm{c}-1}}\left(-, \mathcal{O}_{\mathbb{P}_{R}^{\mathrm{c}-1}}\right)$. It follows from Serre's Vanishing Theorem and the isomorphism (4.12) that for $m \ll 0$, every coherent sheaf $\mathcal{E}$ in $\delta^{\sharp} \mathbb{E} \leq m$ satisfies

$$
\mathrm{H}^{i}\left(\mathbb{P}_{R}^{\mathrm{c}-1}, \mathcal{E}(-c)^{\vee}\right)=0 \text { for all } i \neq 0 .
$$

By [16, 7.9.10], $\mathrm{H}^{0}\left(\mathbb{P}_{R}^{\mathrm{c}-1}, \mathcal{E}(-c)^{\vee}\right)$ is thus a finitely generated projective $R$-module for each such $\mathcal{E}$. By a corollary to Serre-Grothendieck Duality, see [18, III.5.2], we get

$$
\begin{aligned}
\operatorname{Hom}_{R}\left(\mathrm{H}^{i}\left(\mathbb{P}_{R}^{\mathrm{c}-1}, \mathcal{E}(-c)^{\vee}\right), R\right) & \cong \operatorname{Ext}_{\mathcal{O}_{R}^{c-1}}^{c-1-i}\left(\mathcal{E}(-c)^{\vee}, \mathcal{O}(-c)\right) \\
& \cong \operatorname{Ext}_{\mathcal{O}_{\mathbb{P}_{R}^{c-1}}^{c-1-i}}\left(\mathcal{O}_{X}, \mathcal{E}\right) \\
& \cong \mathrm{H}^{c-1-i}\left(\mathbb{P}_{R}^{c-1}, \mathcal{E}\right)
\end{aligned}
$$

for all $\mathcal{E}$ in $\delta^{\sharp} \mathbb{E} \leq m$ and all $i$. The first two assertions follow.

The last part was noted below the definition of $\eta$.

Proposition 4.13. For every object $\mathbb{E}$ of $M F\left(\mathbb{P}_{Q}^{c-1}, \mathcal{O}(1), W\right)$ and integer $m$ that is sufficiently small for $\mathbb{E}$, there is an isomorphism

$$
\zeta_{\mathbb{E}}^{m}: \Gamma\left(\mathcal{U}, \delta^{\sharp} \mathbb{E}\right) \stackrel{\cong}{\longrightarrow} F\left(\left(\delta^{\sharp} \mathbb{E}\right) \leq m\right) \text { in } \mathrm{D}_{\mathrm{sg}}(R)
$$

such that the following naturality condition holds: given a strict map $g: \mathbb{E} \rightarrow \mathbb{F}$ and an integer $n \leq m$ that is sufficiently small for $\mathbb{F}$, the diagram

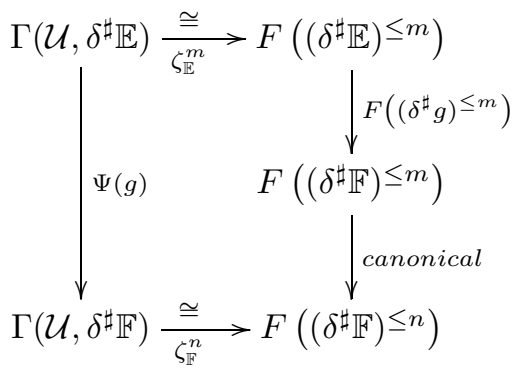

commutes in $\mathrm{D}_{\mathrm{sg}}(R)$.

Proof. Given such a pair $(\mathbb{E}, m)$, define $\zeta_{\mathbb{E}}^{m}$ to be the map in $\mathrm{D}_{\mathrm{sg}}(R)$ represented by the composition of

$$
\Gamma\left(\mathcal{U}, \delta^{\sharp} \mathbb{E}\right) \stackrel{\eta}{\longrightarrow} F\left(\delta^{\sharp} \mathbb{E}\right) \rightarrow F\left(\left(\delta^{\sharp} \mathbb{E}\right) \leq m\right),
$$

where $F\left(\delta^{\sharp} \mathbb{E}\right) \rightarrow F\left(\left(\delta^{\sharp} \mathbb{E}\right) \leq m\right)$ is induced by the canonical map $\delta^{\sharp} \mathbb{E} \rightarrow\left(\delta^{\sharp} \mathbb{E}\right) \leq m$.

The naturality of $\eta$ shows that the diagram

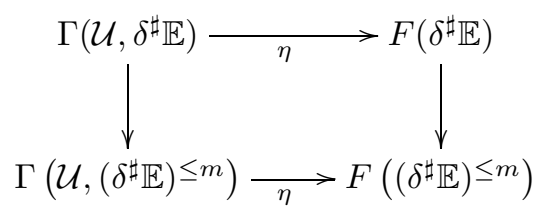


commutes, where the vertical maps are induced by $\delta^{\sharp} \mathbb{E} \rightarrow \delta^{\sharp} \mathbb{E} \leq m$. Thus to show that (4.14) is an isomorphism it is enough to show that the left vertical map and the lower horizontal map in (4.15) are isomorphisms.

The lower horizontal map of (4.15) is an isomorphism in $\mathrm{D}_{\mathrm{sg}}(R)$ by Lemma 4.11. The cone of $\delta^{\sharp}(\mathbb{E}) \rightarrow \delta^{\sharp}(\mathbb{E}) \leq m$ is a bounded complex of locally free coherent sheaves, i.e. is a perfect complex, and $\mathbf{R} \pi_{*}=\Gamma(\mathcal{U},-)$ maps perfect complexes to perfect complexes by [1, III.4.8.1]. Thus the left vertical map of (4.15) is an isomorphism in $\mathrm{D}_{\mathrm{sg}}(R)$.

The naturality assertion is evident from the construction.

Remark 4.16. By (4.9) and the construction of $\zeta$ above, we see that there is a commutative diagram

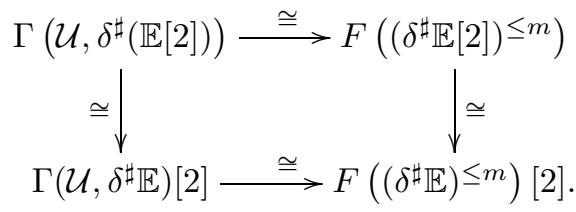

4.1. Recollection of Eisenbud operators. We recall from [15, §1] the construction of the Eisenbud operators for the commutative ring $R=Q /(\mathbf{f})$, where $\mathbf{f}=\left(f_{1}, \ldots, f_{c}\right)$ is a $Q$-regular sequence. Note that the $\operatorname{ring} Q$ need not be regular for this construction.

Let $F$ be a complex of projective $R$-modules such that there exists a sequence of maps of projective $Q$-modules

$$
\widetilde{F}=\cdots \rightarrow \widetilde{F}^{p-1} \stackrel{\tilde{\partial}^{p-1}}{\longrightarrow} \widetilde{F}^{p} \stackrel{\tilde{\partial}^{p}}{\longrightarrow} \widetilde{F}^{p+1} \rightarrow \cdots
$$

with $F \cong \widetilde{F} \otimes_{Q} R$. The sequence of maps $\widetilde{F}$ is not required to be a chain complex. Since $\widetilde{F} \otimes_{Q} R \cong F$, for each $i=1, \ldots, c$ and all $n$, there exist (non-unique) maps $\tilde{t}_{i}^{n}: \widetilde{F}^{n} \rightarrow \widetilde{F}^{n+2}$ such that

$$
\tilde{\partial}^{n+1} \tilde{\partial}^{n}=\sum_{i=1}^{c} \tilde{t}_{i}^{n} f_{i} .
$$

Set $t_{i}^{n}=\tilde{t}_{i}^{n} \otimes_{Q} R: F^{n} \rightarrow F^{n+2}$. Then the following properties hold [15, Section 1]:

(1) for each $i$, the maps $t_{i}^{n}$ assemble to give morphisms of chain complexes, $t_{i}: F \rightarrow F[2]$

(2) the chain map $t_{i}$ is independent of the choice of $\widetilde{F}$ and the $\tilde{t}_{i}^{n}$ 's, up to homotopy;

(3) the $t_{i}$ 's commute, up to homotopy;

(4) the $t_{i}$ 's are natural, up to homotopy, in the argument $F$.

It is assumed in 15 that $F$ is a complex of free $R$-modules, in which case a lifting $\widetilde{F}$ always exists: viewing the differentials of $F$ as matrices, simply lift each element in each matrix to an element of $Q$. However, if $F$ is only assumed to be a complex of projective modules for which such a lifting exists, then the proofs in [15] go through unchanged.

For any object $M$ in $\mathrm{D}^{\mathrm{b}}(R)$ we may choose a complex of projective $R$-modules $F$, an isomorphism $\alpha: F \stackrel{\cong}{\longrightarrow} M \in \mathrm{D}^{\mathrm{b}}(R)$, and a lifting $\widetilde{F}$ of $F$ as above. For example, one could take $F$ to be a bounded above complex of free modules that maps via a 
quasi-isomorphism to $M$. Define $t_{i}^{M}: M \rightarrow M[2]$ in $\mathrm{D}^{\mathrm{b}}(R)$ to be the composition of

$$
M \stackrel{\alpha^{-1}}{\longrightarrow} F \stackrel{t_{i}}{\longrightarrow} F[2] \stackrel{\alpha[2]}{\longrightarrow} M[2],
$$

where the map $t_{i}$ is induced from a choice of maps $t_{i}^{n}$ satisfying (4.18).

The facts listed above imply that $t_{i}^{M}$ does not depend on the choice of $F$ or $t_{i}$, that $t_{i}^{M} \circ t_{j}^{M}=t_{j}^{M} \circ t_{i}^{M}$ for all $i, j$ and, if $g: M \rightarrow N$ is a morphism in $\mathrm{D}^{\mathrm{b}}(R)$, then

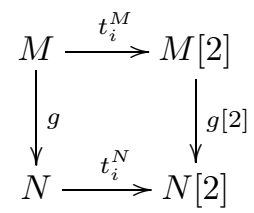

commutes. In other words, the collection of maps $t_{i}^{M}$ form a family of pairwise commuting natural transformations from the identity functor to the functor $(-)[2]$. We call these the Eisenbud operators on $\mathrm{D}^{\mathrm{b}}(R)$ given by $(Q, \mathbf{f})$.

It is clear that $t_{i}^{M}$ descends along the canonical functor $\mathrm{D}^{\mathrm{b}}(R) \rightarrow \mathrm{D}_{\mathrm{sg}}(R)$ to give natural transformations on $\mathrm{D}_{\mathrm{sg}}(R)$, and we will also write these induced transformations as $t_{i}^{M}$. Moreover, these induced transformations, in turn, restrict to give natural transformations of endo-functors on the full, triangulated subcategory $\mathrm{D}_{\mathrm{sg}}^{\mathrm{rel}}(Q \rightarrow R)$ of $\mathrm{D}_{\mathrm{sg}}(R)$.

Proof of Theorem 3.2 . Let $\mathbb{E}$ be an object of $\left[M F\left(\mathbb{P}_{Q}^{\mathrm{c}-1}, \mathcal{O}(1), W\right)\right]$. Let $m$ be an integer which is sufficiently small for $\mathbb{E}[2]$; by definition it will also be sufficiently small for $\mathbb{E}$. By Lemma 4.11 and Proposition 4.13, $\zeta^{m}: \Gamma\left(\mathcal{U}, \delta^{\sharp} \mathbb{E}\right) \rightarrow F\left(\left(\delta^{\sharp} \mathbb{E}\right) \leq m\right)$ is an isomorphism in $\mathrm{D}_{\mathrm{sg}}(R)$ and $F\left(\left(\delta^{\sharp} \mathbb{E}\right) \leq m\right)$ is a complex of finitely generated projective $R$-modules. Using the isomorphism of functors $\Psi(-) \cong \Gamma\left(\mathcal{U}, \delta^{\sharp}-\right)$ of Proposition 4.5, we have a commutative diagram:

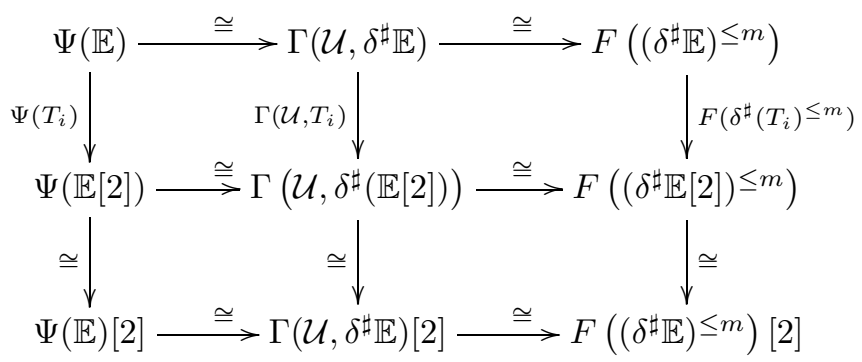

Indeed, the top left square commutes since it is given by a natural transformation applied to the map $T_{i}$. The top right square commutes by Proposition 4.13 , The lower left square commutes since $\Psi(-) \cong \Gamma\left(\mathcal{U}, \delta^{\sharp}-\right)$ is an isomorphism of triangulated functors, and the lower right square commutes by (4.17).

Since $F\left(\left(\delta^{\sharp} \mathbb{E}\right) \leq m\right)$ is a complex of projective $R$-modules, we may calculate Eisenbud operators using it. Let us call the right-hand vertical map in the above diagram $\beta_{i}: F\left(\left(\delta^{\sharp} \mathbb{E}\right) \leq m\right) \rightarrow F\left(\left(\delta^{\sharp} \mathbb{E}\right) \leq m\right)[2]$. To prove Theorem 3.2 it is enough to find a lifting of $F\left(\left(\delta^{\sharp} \mathbb{E}\right) \leq m\right)$ to a complex of projective $Q$-modules and degree -2 endomorphisms $\tilde{t}_{i}$ of the lifting such that (4.18) holds, and such that $\tilde{t}_{i} \otimes_{Q} R \cong \beta_{i}$. 
To lift $F\left(\left(\delta^{\sharp} \mathbb{E}\right) \leq m\right)$ to a sequence of projective $Q$-modules consider the sequence of maps of $Q$-modules defined in analogy with Definition 4.6.

$$
\begin{aligned}
\widetilde{F}(\mathbb{E}):= & \left(\cdots \rightarrow \mathrm{H}^{c-1}\left(\mathbb{P}_{Q}^{c-1}, \mathcal{E}_{0}(-2)\right) \rightarrow \mathrm{H}^{c-1}\left(\mathbb{P}_{Q}^{c-1}, \mathcal{E}_{1}(-1)\right)\right. \\
& \left.\rightarrow \mathrm{H}^{c-1}\left(\mathbb{P}_{Q}^{c-1}, \mathcal{E}_{0}(-1)\right) \rightarrow 0\right),
\end{aligned}
$$

with $\mathrm{H}^{c-1}\left(\mathbb{P}_{Q}^{c-1}, \mathcal{E}_{0}(-q)\right)$ located in cohomological degree $2 c-2$. Just as for $F\left(\left(\delta^{\sharp} \mathbb{E}\right) \leq m\right)$, if $m \ll 0$, each coherent sheaf in $\widetilde{F}(\mathbb{E})^{\leq m}$ is a finitely generated projective $Q$-module (but this is not a chain complex). We claim there is an isomorphism of chain complexes

$$
\widetilde{F}(\mathbb{E})^{\leq m} \otimes_{Q} R \cong F\left(\left(\delta^{\sharp} \mathbb{E}\right) \leq m\right)
$$

for all $m \ll 0$. Indeed, if $\mathcal{E}$ is any coherent sheaf on $\mathbb{P}_{Q}^{\mathrm{c}-1}$, then by [18, II.5.12] we have the isomorphism $\mathbf{R} \pi_{*} \mathbf{L} \delta^{*} \mathcal{E} \cong R \otimes_{Q}^{\mathbf{L}} \mathbf{R} p_{*} \mathcal{E}$ in $\mathrm{D}^{\mathrm{b}}(R)$, where $p: \mathbb{P}_{Q}^{\mathrm{c}-1} \rightarrow \operatorname{Spec} Q$ is the canonical map. Moreover, if $\mathcal{E}$ is locally free and $\mathrm{H}^{i}\left(\mathbb{P}_{Q}^{\mathrm{c}-1}, \mathcal{E}\right)=0$ for all $i \neq c-1$, then this gives an isomorphism of $R$-modules

$$
\mathrm{H}^{c-1}\left(\mathbb{P}_{R}^{\mathrm{c}-1}, \delta^{*} \mathcal{E}\right) \cong \mathrm{H}^{c-1}\left(\mathbb{P}_{Q}^{\mathrm{c}-1}, \mathcal{E}\right) \otimes_{Q} R,
$$

and the claim follows.

Thus $\widetilde{F}(\mathbb{E})^{\leq m}$ is a lifting; to see it is the lifting we sought, note that $\beta_{i}$ is given in each degree by

$$
\mathrm{H}^{c-1}\left(\mathbb{P}_{R}^{\mathrm{c}-1}, \mathcal{E}_{j}(k)\right) \stackrel{\mathrm{H}^{c-1}\left(\mathbb{P}_{R}^{\mathrm{c}-1}, T_{i}\right)}{\longrightarrow} \mathrm{H}^{c-1}\left(\mathbb{P}_{R}^{\mathrm{c}-1}, \mathcal{E}_{j}(k+1)\right) .
$$

The composition of two successive maps in $\widetilde{F}(\mathbb{E}) \leq m$ is the map on sheaf cohomology induced by multiplication by $\sum_{i=1}^{c} T_{i} f_{i}$. Thus, letting $\tilde{t}_{i}=\mathrm{H}^{c-1}\left(\mathbb{P}_{Q}^{c-1}, T_{i}\right)$ we see that $\beta_{i}=\tilde{t}_{i} \otimes_{Q} R$ and the theorem follows.

\section{Projective Resolutions}

We continue to work in the context and under the assumptions of 92.3 , with the added assumption that $Q$ is Gorenstein, i.e. $Q$ has finite injective dimension over itself. Let $M$ be a finitely generated $R$-module that has finite projective dimension over $Q$. In this section we construct a projective resolution over $R$ of a high syzygy of $M$ using the equivalence $\Psi$ of Corollary 2.11 and the explicit representative of $\Psi(\mathbb{E})$ given in Section 4

Recall that a coherent sheaf $\mathcal{F}$ on $\mathbb{P}_{R}^{\mathrm{c}-1}$ is $m$-regular for an integer $m$ if

$$
\mathrm{H}^{i}\left(\mathbb{P}_{R}^{\mathrm{c}-1}, \mathcal{F}(m-i)\right)=0 \text { for all } i>0 .
$$

The regularity of $\mathcal{F}$ is the smallest $m$ such that $\mathcal{F}$ is $m$-regular; see e.g. 23 , Lecture 14] and [30, Section 8]. By Serre's Vanishing Theorem, every coherent sheaf on $\mathbb{P}_{R}^{\mathrm{c}-1}$ has finite regularity.

Definition 5.1. For a matrix factorization $\mathbb{E}=\left(\mathcal{E}_{1} \rightarrow \mathcal{E}_{0} \rightarrow \mathcal{E}_{1}(1)\right)$, we set

$$
\alpha(\mathbb{E})=\max \left\{\text { regularity }\left(\delta^{*} \mathcal{E}_{0}\right)^{\vee}-1 \text {, regularity }\left(\delta^{*} \mathcal{E}_{1}\right)^{\vee}-1\right\},
$$

where $\delta: \mathbb{P}_{R}^{c-1} \rightarrow \mathbb{P}_{Q}^{c-1}$ is the canonical inclusion, and $(-)^{\vee}=\mathcal{H}$ om $(-, \mathcal{O})$. 
Theorem 5.2. Let $R=Q /\left(f_{1}, \ldots, f_{c}\right)$, where $Q$ is a Gorenstein ring of finite Krull dimension and $f_{1}, \ldots, f_{c}$ is a $Q$-regular sequence. Let $M$ be a finitely generated $R$ module that has finite projective dimension over $Q$ and let $\mathbb{E}=\left(\mathcal{E}_{1} \rightarrow \mathcal{E}_{0} \rightarrow \mathcal{E}_{1}(1)\right)$ be an object of $M F\left(\mathbb{P}_{Q}^{c-1}, \mathcal{O}(1), W\right)$ such that $\Psi(\mathbb{E}) \cong M$, where $\Psi$ is the equivalence of Corollary 2.11. Set

$$
n_{\mathbb{E}}=2 \alpha(\mathbb{E})+e-1,
$$

where $e$ is the is the Krull dimension of $Q$. The complex

$$
\begin{aligned}
F\left(\delta^{\sharp} \mathbb{E}\right)^{\leq-n_{\mathbb{E}}}=\cdots & \rightarrow \mathrm{H}^{c-1}\left(\mathbb{P}_{R}^{c-1}, \delta^{*} \mathcal{E}_{1}(m)\right) \rightarrow \mathrm{H}^{c-1}\left(\mathbb{P}_{R}^{c-1}, \delta^{*} \mathcal{E}_{0}(m)\right) \\
& \rightarrow \mathrm{H}^{c-1}\left(\mathbb{P}_{R}^{c-1}, \delta^{*} \mathcal{E}_{1}(m+1)\right) \rightarrow \cdots
\end{aligned}
$$

is a projective resolution of an $n_{\mathbb{E}}$-th syzygy of $M$, where $\delta^{\sharp} \mathbb{E}$ is defined in Definition 4.1 and $F(-)$ is defined in Definition 4.6 .

Remark 5.3. On this resolution one may choose the Eisenbud operators

$$
t_{i}: \mathrm{H}^{c-1}\left(\mathbb{P}_{R}^{\mathrm{c}-1}, \delta^{*} \mathcal{E}_{i}(n)\right) \rightarrow \mathrm{H}^{c-1}\left(\mathbb{P}_{R}^{\mathrm{c}-1}, \delta^{*} \mathcal{E}_{i}(n+1)\right)
$$

to be multiplication by $T_{i} \in \Gamma\left(\mathbb{P}_{Q}^{c-1}, \mathcal{O}_{\mathbb{P}_{Q}^{c-1}}(1)\right)$. In particular, these operators commute.

We need several preliminary results for the proof of Theorem 5.2 .

Lemma 5.4. The integer $-2 \alpha(\mathbb{E})-c+1$ is sufficiently small for the matrix factorization $\mathbb{E}$, in the sense of Definition 4.10 .

Proof. By [30, 8.1.3], if a sheaf $\mathcal{F}$ on $\mathbb{P}_{R}^{\mathrm{c}-1}$ is $k$-regular, then it is also $(k+1)$-regular. Thus the sheaves $\left(\delta^{*} \mathcal{E}_{0}\right)^{\vee}$ and $\left(\delta^{*} \mathcal{E}_{1}\right)^{\vee}$ are $k$-regular for all $k>\alpha(\mathbb{E})$. In particular,

$\mathrm{H}^{i}\left(\mathbb{P}_{R}^{\mathrm{c}-1},\left(\delta^{*} \mathcal{E}_{0}\right)^{\vee}(k)\right)=0=\mathrm{H}^{i}\left(\mathbb{P}_{R}^{\mathrm{c}-1},\left(\delta^{*} \mathcal{E}_{1}\right)^{\vee}(k)\right)$ for all $i>0$ and all $k \geq \alpha(\mathbb{E})$.

As in the proof of Lemma 4.11, this implies that for $j=0,1$ and all $k \geq \alpha(\mathbb{E})$ :

(1) the $R$-module $\mathrm{H}^{0}\left(\mathbb{P}_{R}^{\mathrm{c}-1},\left(\delta^{*} \mathcal{E}_{j}\right)^{\vee}(k)\right)$ is projective;

(2) there is an isomorphism

$$
\operatorname{Hom}_{R}\left(\mathrm{H}^{0}\left(\mathbb{P}_{R}^{\mathrm{c}-1},\left(\delta^{*} \mathcal{E}_{j}\right)^{\vee}(k)\right), R\right) \cong \mathrm{H}^{c-1}\left(\mathbb{P}_{R}^{\mathrm{c}-1},\left(\delta^{*} \mathcal{E}_{j}\right)(-k-c)\right),
$$

and so in particular $\mathrm{H}^{c-1}\left(\mathbb{P}_{R}^{\mathrm{c}-1},\left(\delta^{*} \mathcal{E}_{j}\right)(-k-c)\right)$ is projective;

(3) $\mathrm{H}^{i}\left(\mathbb{P}_{R}^{\mathrm{c}-1},\left(\delta^{*} \mathcal{E}_{j}\right)(-k-c)\right)=0$ for all $i<c-1$.

Now consider the complex

$$
\delta^{\sharp} \mathbb{E}:=\left(\cdots \rightarrow \delta^{*} \mathcal{E}_{0}(-2) \rightarrow \delta^{*} \mathcal{E}_{1}(-1) \rightarrow \delta^{*} \mathcal{E}_{0}(-1) \rightarrow 0\right)
$$

where $\delta^{*} \mathcal{E}_{0}(-n)$ is in cohomological degree $c-2 n+1$. We have

$$
\begin{aligned}
\left(\delta^{\sharp} \mathbb{E}\right) & \leq-2 \alpha(\mathbb{E})-c+1:=\left(\cdots \rightarrow \delta^{*} \mathcal{E}_{0}(-(\alpha(\mathbb{E})+1)-c)\right. \\
& \left.\rightarrow \delta^{*} \mathcal{E}_{1}(-\alpha(\mathbb{E})-c) \rightarrow \delta^{*} \mathcal{E}_{0}(-\alpha(\mathbb{E})-c) \rightarrow 0\right),
\end{aligned}
$$

and by the above each sheaf appearing in this complex satisfies the two conditions of Definition 4.10 .

Proposition 5.5. The cohomology of the complex $F\left(\delta^{\sharp} \mathbb{E}\right) \leq-2 \alpha(\mathbb{E})$ is concentrated in degrees $-2 \alpha(\mathbb{E})-c+1 \leq i \leq-2 \alpha(\mathbb{E})$. 
Proof. From the definition of $F$ we have that $F\left(\delta^{\sharp} \mathbb{E}\right) \leq-2 \alpha(\mathbb{E})=F\left(\left(\delta^{\sharp} \mathbb{E}\right) \leq-2 \alpha(\mathbb{E})-c+1\right)$. Set $n=-2 \alpha(\mathbb{E})-c+1$. By Lemma 5.4, $n$ is sufficiently small for $\mathbb{E}$, and thus by Lemma 4.11 there is an isomorphism in $\mathrm{D}^{\mathrm{b}}(R)$ :

$$
F\left(\left(\delta^{\sharp} \mathbb{E}\right)^{\leq n}\right) \cong \Gamma\left(\mathcal{U},\left(\delta^{\sharp} \mathbb{E}\right)^{\leq n}\right) .
$$

By Remark 4.2 the complex of sheaves $\delta^{\sharp} \mathbb{E} \leq n$ only has cohomology in degree $n$. Setting $\mathcal{N}=\mathrm{H}^{n}\left(\delta^{\sharp} \mathbb{E} \leq n\right)$ gives an isomorphism in $\mathrm{D}^{\mathrm{b}}(Y)$ :

$$
\delta^{\sharp} \mathbb{E}^{\leq n} \cong \mathcal{N}[-n] .
$$

Since the functor $\Gamma(\mathcal{U},-)$ represents $\mathbf{R} \pi_{*}$, we have the following isomorphisms in $\mathrm{D}^{\mathrm{b}}(R)$ :

$$
\Gamma\left(\mathcal{U}, \delta^{\sharp}(\mathbb{E}) \leq n\right) \cong \mathbf{R} \pi_{*}\left(\delta^{\sharp}(\mathbb{E}) \leq n\right) \cong \mathbf{R} \pi_{*}(\mathcal{N}[-n]) \cong \mathbf{R} \pi_{*}(\mathcal{N})[-n] .
$$

Since $\mathbf{R} \pi_{*}$ has cohomological dimension $c-1$, we see that $\mathbf{R} \pi_{*}(\mathcal{N})$ has cohomology in degrees at most $0 \leq i \leq c-1$. Combining the above gives that

$$
\begin{aligned}
F\left(\delta^{\sharp} \mathbb{E}\right) \leq-2 \alpha(\mathbb{E}) & =F\left(\delta^{\sharp}(\mathbb{E})^{\leq n}\right) \\
& \cong \Gamma\left(\mathcal{U}, \delta^{\sharp}(\mathbb{E})^{\leq n}\right) \\
& \cong \mathbf{R} \pi_{*}(\mathcal{N})[-n]
\end{aligned}
$$

has cohomology in degrees at most $n \leq i \leq n+c-1$.

Proof of Theorem 5.2. Let $M$ be a finitely generated $R$-module with finite projective dimension over $Q$, and let $\mathbb{E}$ a matrix factorization such that $\Psi(\mathbb{E}) \cong M \in$ $\mathrm{D}_{\mathrm{sg}}(R)$. Since $-2 \alpha(\mathbb{E})-c+1$ is sufficiently small for $\mathbb{E}$ by Lemma 5.4, there is, by Propositions 4.5 and 4.13 an isomorphism in $\mathrm{D}_{\mathrm{sg}}(R)$,

$$
\Psi(\mathbb{E}) \stackrel{\cong}{\longrightarrow} F\left(\delta^{\sharp}(\mathbb{E}) \leq-2 \alpha(\mathbb{E})-c+1\right)=F\left(\delta^{\sharp} \mathbb{E}\right) \leq-2 \alpha(\mathbb{E}) .
$$

Thus we have that

$$
M \cong F\left(\delta^{\sharp} \mathbb{E}\right)^{\leq-2 \alpha(\mathbb{E})} \in \mathrm{D}_{\mathrm{sg}}(R) .
$$

By Proposition [5.5. the complex $F\left(\delta^{\sharp} \mathbb{E}\right) \leq-2 \alpha(\mathbb{E})-c+1$ only has cohomology in degree $-2 \alpha(\mathbb{E})-c+1$. Moreover, since $F\left(\delta^{\sharp} \mathbb{E}\right)$ is a complex of projective modules, the cone of the canonical map

$$
F\left(\delta^{\sharp} \mathbb{E}\right) \leq-2 \alpha(\mathbb{E}) \rightarrow F\left(\delta^{\sharp} \mathbb{E}\right) \leq-2 \alpha(\mathbb{E})-c+1
$$

is perfect, and hence (5.6) is an isomorphism in $\mathrm{D}_{\mathrm{sg}}(R)$. Finally, since $R$ is CohenMacaulay, we may truncate $F\left(\delta^{\sharp} \mathbb{E}\right) \leq-2 \alpha(\mathbb{E})-c+1$ to the left $d=\operatorname{dim} R$ more steps so that the module

$$
N=\mathrm{H}^{-2 \alpha(\mathbb{E})-c-d+1}\left(F\left(\delta^{\sharp} \mathbb{E}\right) \leq-2 \alpha(\mathbb{E})-c-d+1\right)
$$

is maximal Cohen-Macaulay (MCM). Note also that $c+d$, where $c$ is the length of the regular sequence defining $R$, is exactly the Krull dimension of $Q$, which we called $e$. Thus $-2 \alpha(\mathbb{E})-c-d+1=-n_{\mathbb{E}}$, which was defined above.

We now have an isomorphism

$$
M \cong N\left[-n_{\mathbb{E}}\right] \in \mathrm{D}_{\mathrm{sg}}(R) .
$$

Let $P=\cdots \rightarrow P^{-m} \stackrel{\partial_{P}^{-m}}{\longrightarrow} P^{m+1} \rightarrow \cdots \rightarrow P^{-1} \stackrel{\partial_{P}^{-1}}{\longrightarrow} P^{0}$ be a projective resolution of $M$ over $R$. By induction on $n$ one checks that there are isomorphisms for all $m \geq 0$ :

$$
M \cong\left(\operatorname{coker} \partial_{P}^{-m}\right)[m-1] \in \mathrm{D}_{\mathrm{sg}}(R)
$$


Setting $M^{\prime}=$ coker $\partial_{P}^{-n_{\mathbb{E}}-1}$, which is MCM since $n_{\mathbb{E}} \geq d$, we have that

$$
M^{\prime} \cong N \in \mathrm{D}_{\mathrm{sg}}(R) \text {. }
$$

By [11, 4.4.1] this implies that $M^{\prime} \cong N$ in the stable category of MCM modules, i.e. there exist projective $R$-modules $Q_{1}, Q_{2}$ and an isomorphism of $R$-modules

$$
M^{\prime} \oplus Q_{1} \cong N \oplus Q_{2} .
$$

This allows us to glue the acyclic complexes

$$
\begin{gathered}
\cdots \rightarrow F\left(\delta^{\sharp} \mathbb{E}\right)^{-n_{\mathbb{E}}-1} \rightarrow F\left(\delta^{\sharp} \mathbb{E}\right)^{-n_{\mathbb{E}}} \rightarrow N \rightarrow 0, \\
0 \rightarrow M^{\prime} \rightarrow P^{-n_{\mathbb{E}}+1} \rightarrow P^{-n_{\mathbb{E}}+2} \rightarrow \cdots \rightarrow P^{-1} \rightarrow P^{0} \rightarrow M \rightarrow 0
\end{gathered}
$$

to give a projective resolution of $M$.

\section{A DESCRIPTION OF $\Psi^{-1}$ ON OBJECTS}

We work in the context and under the assumptions of $\$ 2.3$. Let $M$ be a finitely generated $R$-module that has finite projective dimension over $Q$. Our goal in this section is to give an explicit method of constructing a matrix factorization $\mathbb{E}_{M}$ such that $\Psi\left(\mathbb{E}_{M}\right) \cong M$ holds in $\mathrm{D}_{\mathrm{sg}}(R)$, where $\Psi$ is the equivalence of Corollary 2.11. We achieve this using the data of a projective resolution of $M$ over $Q$ and a system of "higher homotopies" on this resolution, as introduced by Eisenbud in [15.

6.1. Standard resolutions. Let $G=0 \rightarrow G_{n} \rightarrow \cdots \rightarrow G_{0} \rightarrow 0$ be a resolution of $M$ by finitely generated projective $Q$-modules.

For an element $J=\left(a_{1}, \ldots, a_{c}\right) \in \mathbb{N}^{c}$, we set $|J|=\sum_{1}^{c} a_{i}$. We write the element $(0, \ldots, 1, \ldots, 0)$, where the 1 is in the $i$-th position, as $i$. By [15, 7.1] there exists a family of endomorphisms of $G$

$$
\boldsymbol{\sigma}=\left\{\sigma^{J} \mid J \in \mathbb{N}^{c}\right\}
$$

where $\sigma^{J} \in \boldsymbol{\sigma}$ has degree $2|J|-1$ (i.e. the components are $\sigma_{j}^{J}: G_{j} \rightarrow G_{j+2|J|-1}$ ) that satisfy the following equations:

$$
\begin{aligned}
\sigma^{0} & =\partial_{G}, \\
\sigma^{0} \sigma^{i}+\sigma^{i} \sigma^{0} & =f_{i} 1_{G}, \\
\sum_{J^{\prime}+J^{\prime \prime}=J} \sigma^{J^{\prime}} \sigma^{J^{\prime \prime}} & =0 \text { for all } J \in \mathbb{N}^{c} \text { with }|J| \geq 2 .
\end{aligned}
$$

Such a family $\boldsymbol{\sigma}$ is called a system of higher homotopies.

Remark 6.1. The result above is stated for complexes of free modules in [15, but the proof works under the weaker assumption that the modules are projective.

Example 6.2. Let $K=K\left\langle e_{1}, \ldots, e_{c} \mid \partial\left(e_{i}\right)=f_{i}\right\rangle$ be the Koszul complex resolving $R$ over $Q$. Then $K$ has the structure of a differential graded (DG)-algebra with multiplication given by the alternating product. For an $R$-module $M$ with finite projective dimension over $Q$, we may find a finite projective resolution $G$ of $M$ over $Q$ such that $G$ is a DG-module over $K$ (and such that the $Q$-module structure coincides with the restriction along the canonical map $Q \rightarrow K)$. See [3, Section 2] for an explicit construction of such a $G$. Setting $\sigma^{0}=\partial^{G}, \sigma^{i}$ to be multiplication by $e_{i}$ for $i=1, \ldots, c$, and $\sigma^{J}=0$ whenever $|J| \geq 2$ gives a system of higher homotopies on $G$. While such a system of higher homotopies is simpler than the general type, not every $Q$-projective resolution of $M$ has the structure of a DG-module over $K$; see [2]. 
Define $D=\bigoplus_{j \leq 0} D^{j}=\bigoplus_{j \leq 0} \operatorname{Hom}_{R}\left(R\left[\chi_{1}, \ldots, \chi_{c}\right]_{-j}, R\right)$ to be the graded dual of the polynomial ring $R\left[\chi_{1}, \ldots, \chi_{c}\right]$, where the $\chi_{i}$ 's are indeterminants of degree 2 . We regard $D$ as a graded $R\left[\chi_{1}, \ldots, \chi_{c}\right]$-module in the obvious way. (In fact $D$ is a divided power algebra, but we ignore the multiplication rule and regard it only as a graded $R\left[\chi_{1}, \ldots, \chi_{c}\right]$-module.) Consider the graded $R$-module

$$
\bar{G} \otimes_{R} D,
$$

where $G$ is the projective $Q$-resolution of $M$ above, and $\bar{G}=G \otimes_{Q} R$. Note that $\bar{G}_{i} \otimes_{R} D^{2 j}$ lies in cohomological degree $2 j-i$. Let $\boldsymbol{\sigma}$ be a system of higher homotopies on $G$, and define $\partial=\sum_{J \in \mathbb{N}^{c}} \sigma^{J} \otimes \chi^{J}$, where $\chi^{J}=\chi_{1}^{a_{1}} \ldots \chi_{c}^{a_{c}}$ for $J=\left(a_{1}, \ldots, a_{c}\right) \in$ $\mathbb{N}^{c}$, a degree -1 endomorphism of $\bar{G} \otimes_{R} D$. When $R$ is local, [15, Theorem 7.2] shows that this complex is an $R$-free resolution of $M$. By localizing, we see that, in general, $\bar{G} \otimes_{R} D$ is a projective $R$-resolution of $M$. This is a standard resolution of $M$ which we write as $G\{\boldsymbol{\sigma}\}$.

6.2. Construction of a matrix factorization. Recall that $S$ is the graded $Q$ algebra $S:=Q\left[T_{1}, \ldots, T_{c}\right]$, with $\left|T_{i}\right|=1$, and $W=f_{1} T_{1}+\cdots+f_{c} T_{c} \in S$. Let $S(j)$ denote the graded free $S$-module with $S(j)_{i}=S_{i+j}$, and for a graded $S$-module $E$ we set $E(j)=E \otimes_{S} S(j)$.

Definition 6.3. A graded matrix factorization of $W$ is a pair of graded free $S$ modules $E_{1}, E_{0}$ and maps

$$
E_{1} \stackrel{g_{1}}{\longrightarrow} E_{0} \stackrel{g_{0}(1)}{\longrightarrow} E_{1}(1)
$$

such that $g_{0} \circ g_{1}$ and $g_{1}(1) \circ g_{0}$ are multiplication by $W$.

Note that to any graded matrix factorization of $W$ we may apply $\widetilde{(-)}$ to obtain an object of $\left[M F\left(\mathbb{P}_{Q}^{\mathrm{c}-1}, \mathcal{O}(1), W\right)\right]$.

Given a finitely generated $R$-module $M$ with finite projective dimension over $Q$, pick a finite projective resolution $G$ of $M$ as a $Q$-module, and a system of higher homotopies $\boldsymbol{\sigma}=\left\{\sigma^{J}\right\}$. We define the finitely generated graded projective $S$-modules $E_{1}$ and $E_{0}$ as follows:

$$
E_{1}:=\bigoplus_{j \geq 0} G_{2 j+1} \otimes_{Q} S(j) \text { and } E_{0}:=\bigoplus_{j \geq 0} G_{2 j} \otimes_{Q} S(j) .
$$

For $J \in \mathbb{N}^{c}$, consider the maps

$$
\begin{aligned}
& G_{2 j+1} \otimes S(j) \stackrel{\sigma_{J} \otimes T^{J}}{\longrightarrow} G_{2 j+2|J|} \otimes S(j+|J|), \\
& G_{2 j} \otimes S(j) \stackrel{\sigma_{J} \otimes T^{J}}{\longrightarrow} G_{2 j+2|J|-1} \otimes S(j+|J|) .
\end{aligned}
$$

We use these to define homogeneous maps $g_{1}: E_{1} \rightarrow E_{0}$ and $g_{0}: E_{0} \rightarrow E_{1}(1)$, component-wise, as

$$
\begin{gathered}
\left(g_{1}\right)_{j}=\sum_{J \in \mathbb{N}^{c}} \sigma^{J} \otimes T^{J}: G_{2 j+1} \otimes_{Q} S(j) \rightarrow \bigoplus_{i} G_{2 i} \otimes_{Q} S(i)=E_{0}, \\
\left(g_{0}\right)_{j}=\sum_{J \in \mathbb{N}^{c}} \sigma^{J} \otimes T^{J}: G_{2 j} \otimes_{Q} S(j) \rightarrow \bigoplus_{i} G_{2 i-1} \otimes_{Q} S(i)=E_{1}(1) .
\end{gathered}
$$


Using the defining properties of the system of higher homotopies, one checks:

Lemma 6.4. There are equalities:

$$
\begin{aligned}
g_{0} \circ g_{1} & =\sum_{i=1}^{c} f_{i} \otimes T_{i}=1_{E_{1}} \otimes W, \\
g_{1}(1) \circ g_{0} & =\sum_{i=1}^{c} f_{i} \otimes T_{i}=1_{E_{0}} \otimes W .
\end{aligned}
$$

This shows that $E=E(M, G, \boldsymbol{\sigma}):=\left(E_{1} \stackrel{g_{1}}{\longrightarrow} E_{0} \stackrel{g_{0}}{\longrightarrow} E_{1}(1)\right)$ is a graded matrix factorization of $W$.

Definition 6.5. For $G$ and $\boldsymbol{\sigma}$ as above, we set $\mathbb{E}=\mathbb{E}(M, G, \boldsymbol{\sigma})$ to be the object $E(\widetilde{M, G}, \boldsymbol{\sigma})$ of $\left[M F\left(\mathbb{P}_{Q}^{\mathrm{c}-1}, \mathcal{O}(1), W\right)\right]$. Explicitly, $\mathbb{E}=\left(\mathcal{E}_{1} \stackrel{e_{1}}{\longrightarrow} \mathcal{E}_{0} \stackrel{e_{0}}{\longrightarrow} \mathcal{E}(1)\right)$ with

$$
\begin{array}{cc}
\mathcal{E}_{1}=\widetilde{E_{1}}=\bigoplus_{j \geq 0} G_{2 j+1} \otimes_{Q} \mathcal{O}_{\mathbb{P}_{Q}^{\mathrm{c}-1}}(j) & \mathcal{E}_{0}=\widetilde{E_{0}}=\bigoplus_{j \geq 0} G_{2 j} \otimes_{Q} \mathcal{O}_{\mathbb{P}_{Q}^{\mathrm{c}-1}}(j), \\
e_{1}=\widetilde{g_{1}}: \mathcal{E}_{1} \rightarrow \mathcal{E}_{0} & e_{0}=\widetilde{g_{0}}: \mathcal{E}_{0} \rightarrow \mathcal{E}_{1}(1) .
\end{array}
$$

Proposition 6.6. Let $M$ be a finitely generated $R$-module that has finite projective dimension over $Q$. Let $G$ be a finite projective $Q$-resolution of $M, \sigma$ a system of higher homotopies on $G$, and $\mathbb{E}=\mathbb{E}(M, G, \boldsymbol{\sigma})$ the matrix factorization constructed above. Then the complex $F\left(\delta^{\sharp} \mathbb{E}\right)$, where $\delta^{\sharp} \mathbb{E}$ is defined in Definition 4.1 and $F$ is defined in Definition 4.6, is exactly the standard resolution $G\{\boldsymbol{\sigma}\}$ constructed from $G$ and $\boldsymbol{\sigma}$. In particular, there is an isomorphism in $\mathrm{D}_{\mathrm{sg}}(R)$,

$$
\Psi(\mathbb{E}) \cong M,
$$

where $\Psi:\left[M F\left(\mathbb{P}_{Q}^{c-1}, \mathcal{O}(1), W\right)\right] \rightarrow \mathrm{D}_{\mathrm{sg}}(R)$ is the functor of Corollary 2.11.

Proof. First note that $\delta^{*}\left(G_{k} \otimes_{Q} \mathcal{O}_{\mathbb{P}_{Q}^{\mathrm{c}-1}}(j)\right) \cong \overline{G_{k}} \otimes_{R} \mathcal{O}_{\mathbb{P}_{R}^{\mathrm{c}-1}}(j)$, where $\delta: \mathbb{P}_{R}^{\mathrm{c}-1} \hookrightarrow$ $\mathbb{P}_{Q}^{\mathrm{c}-1}$ is the canonical inclusion. Thus, we have that

$\delta^{\sharp}(\mathbb{E})=\cdots \rightarrow \bigoplus_{j} \bar{G}_{2 j+4} \otimes_{R} \mathcal{O}(j) \rightarrow \bigoplus_{j} \bar{G}_{2 j+3} \otimes_{R} \mathcal{O}(j) \rightarrow \bigoplus_{j} \bar{G}_{2 j+2} \otimes_{R} \mathcal{O}(j) \rightarrow 0$,

where the last term is in cohomological degree $c-1$. Applying the functor $F$ we have

$$
\begin{gathered}
F\left(\delta^{\sharp}(\mathbb{E})\right)=\cdots \rightarrow \mathrm{H}^{c-1}\left(\mathbb{P}_{R}^{\mathrm{c}-1}, \bigoplus_{j} \bar{G}_{2 j+4} \otimes_{R} \mathcal{O}(j)\right) \rightarrow \mathrm{H}^{c-1}\left(\mathbb{P}_{R}^{\mathrm{c}-1}, \bigoplus_{j} \bar{G}_{2 j+3} \otimes_{R} \mathcal{O}(j)\right) \\
\rightarrow \mathrm{H}^{c-1}\left(\mathbb{P}_{R}^{\mathrm{c}-1}, \bigoplus_{j} \bar{G}_{2 j+2} \otimes_{R} \mathcal{O}(j)\right) \rightarrow 0,
\end{gathered}
$$

where the last term is located in cohomological degree $2 c-2$. In general, we have that $F\left(\delta^{\sharp}(\mathbb{E})\right)^{2 i}=\bigoplus_{j} G_{2 j+2(c-i)} \otimes \mathcal{O}(j)$. Now, by [19, Thm. III.5.1], for all $j$, there is a natural isomorphism

$$
\begin{aligned}
\mathrm{H}^{c-1}\left(\mathbb{P}_{R}^{\mathrm{c}-1}, \mathcal{O}_{\mathbb{P}_{R}^{\mathrm{c}-1}}(j)\right) & \cong \operatorname{Hom}_{R}\left(R\left[T_{1}, \ldots, T_{c}\right]^{-j-c}, R\right) \\
& \cong \operatorname{Hom}_{R}\left(R\left[\chi_{1}, \ldots, \chi_{c}\right]^{-2 j-2 c}, R\right)=D^{2 j+2 c} .
\end{aligned}
$$


Since each $G_{i}$ is a projective $R$-module, this gives that

$$
\begin{aligned}
F\left(\delta^{\sharp}(\mathbb{E})\right) \cong \cdots & \rightarrow \bigoplus_{j} \bar{G}_{2 j+4} \otimes_{R} D^{2 j+2 c} \rightarrow \bigoplus_{j} \bar{G}_{2 j+3} \otimes_{R} D^{2 j+2 c} \\
& \rightarrow \bigoplus_{j} \bar{G}_{2 j+2} \otimes_{R} D^{2 j+2 c} \rightarrow 0,
\end{aligned}
$$

where the last term is in cohomological degree $2 c-2$. However, $D^{j}=0$ unless $j \leq 0$ and $G_{i}=0$ unless $i \geq 0$, and thus the first non-zero terms of this complex are

$$
\cdots \rightarrow\left(G_{2} \otimes D^{0}\right) \oplus\left(G_{0} \otimes D^{2}\right) \rightarrow G_{1} \otimes D^{0} \rightarrow G_{0} \otimes D^{0} \rightarrow 0,
$$

where $G_{0} \otimes D^{0}$ is in degree 0 . As a graded $R$-module this is exactly $\bar{G} \otimes D$, and one may readily verify that the differentials are the same.

Thus $F\left(\delta^{\sharp}(\mathbb{E})\right)$ is a free resolution of $M$ and so is isomorphic to $M$ in $\mathrm{D}^{\mathrm{b}}(R)$. Let $m$ be an integer which is sufficiently small for $\mathbb{E}$. Then by Proposition 4.13 we have that $F\left(\delta^{\sharp}(\mathbb{E})\right)^{\leq m-c+1}=F\left(\delta^{\sharp}(\mathbb{E}) \leq m\right) \cong \Psi(\mathbb{E}) \in \mathrm{D}_{\mathrm{sg}}(R)$. Since $F\left(\delta^{\sharp}(\mathbb{E})\right)$ is projective in each degree, the canonical map

$$
F\left(\delta^{\sharp}(\mathbb{E})\right) \rightarrow F\left(\delta^{\sharp}(\mathbb{E})\right) \leq m-c+1
$$

is an isomorphism in $\mathrm{D}_{\mathrm{sg}}(R)$. Thus $M \cong \Psi(\mathbb{E}) \in \mathrm{D}_{\mathrm{sg}}(R)$.

\section{Properties of matrix factorizations}

In this section we state and prove basic isomorphisms using matrix factorizations and discuss the support of a matrix factorization. These properties will translate directly to the properties of stable support sets described in Theorem 4 of the Introduction.

In this section, as there is nothing gained in working over $\mathbb{P}_{Q}^{\mathrm{c}-1}$, we work in the generality that $X$ is a Noetherian separated scheme and $\mathcal{L}$ is a line bundle on $X$. As a matter of convenience, we write $\mathcal{F}(1)$ for $\mathcal{F} \otimes_{\mathcal{O}_{X}} \mathcal{L}$ even though $\mathcal{L}$ is not assumed to be very ample.

Definition 7.1. Suppose $W$ and $V$ are global sections of $\mathcal{L}$ and let $\mathbb{E}=\left(\mathcal{E}_{1} \stackrel{e_{1}}{\longrightarrow}\right.$ $\left.\mathcal{E}_{0} \stackrel{e_{0}}{\longrightarrow} \mathcal{E}_{1}(1)\right)$ and $\mathbb{F}=\left(\mathcal{F}_{1} \stackrel{f_{1}}{\longrightarrow} \mathcal{F}_{0} \stackrel{f_{0}}{\longrightarrow} \mathcal{F}_{1}(1)\right)$ be objects of $M F(X, \mathcal{L}, W)$ and $M F(X, \mathcal{L}, V)$, respectively.

(1) Their tensor product is the object $\mathbb{E} \otimes_{M F} \mathbb{F}$ of $M F(X, \mathcal{L}, W+V)$ given by

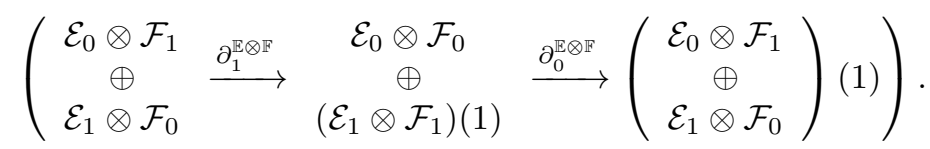

The differentials are given by the formulas

$\partial_{1}^{\mathbb{E} \otimes \mathbb{F}}=\left[\begin{array}{cc}1 \otimes f_{1} & e_{1} \otimes 1 \\ e_{0} \otimes 1 & -1 \otimes f_{0}\end{array}\right] \quad$ and $\quad \partial_{0}^{\mathbb{E} \otimes \mathbb{F}}=\left[\begin{array}{cc}1 \otimes f_{0} & \left(e_{1} \otimes 1\right)(1) \\ e_{0} \otimes 1 & \left(-1 \otimes f_{1}\right)(1)\end{array}\right]$

using the canonical isomorphisms

$$
\left(\begin{array}{c}
\mathcal{E}_{0} \otimes \mathcal{F}_{1} \\
\oplus \\
\mathcal{E}_{1} \otimes \mathcal{F}_{0}
\end{array}\right)(1) \cong \begin{gathered}
\left(\mathcal{E}_{0} \otimes \mathcal{F}_{1}\right)(1) \\
\oplus \\
\left(\mathcal{E}_{1} \otimes \mathcal{F}_{0}\right)(1)
\end{gathered}
$$

and $\mathcal{E}_{i}(1) \otimes \mathcal{F}_{j} \cong\left(\mathcal{E}_{i} \otimes \mathcal{F}_{j}\right)(1) \cong \mathcal{E}_{i} \otimes \mathcal{F}_{j}(1)$. 
(2) Their Hom-object is the object $\mathcal{H o m}_{\mathrm{MF}}(\mathbb{E}, \mathbb{F})$ of $M F(X, \mathcal{L}, V-W)$ given by

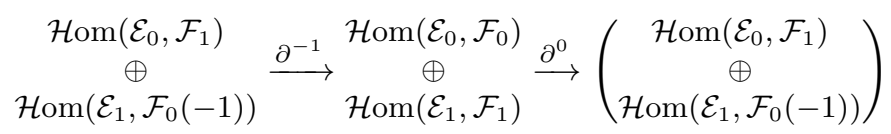

with the differentials defined using the same formulas as in Definition 2.3, which represents the special case $W=V$.

Remark 7.2. We will eventually assume $X$ is regular. If $X$ is not regular, these definitions should be viewed as "non-derived".

Remark 7.3. If $W+V=0$, we may interpret $\mathbb{E} \otimes_{M F} \mathbb{F}$ as an object of $T P C(X, \mathcal{L})$, i.e. a twisted periodic complex. See Definition 2.2 for the definition. If $W=V=0$, we obtain a tensor operator for the category $T P C(X, \mathcal{L})$.

Proposition 7.4. Let $W, V$, and $U$ be global sections of $\mathcal{L}$, and $\mathbb{E}, \mathbb{F}$, and $\mathbb{G}$ be objects of $M F(X, \mathcal{L}, W), M F(X, \mathcal{L}, V)$, and $M F(X, \mathcal{L}, U)$, respectively. There are isomorphisms:

(1) $\mathbb{E} \otimes_{M F} \mathbb{F} \cong \mathbb{F} \otimes_{M F} \mathbb{E}$ in $M F(X, \mathcal{L}, W+V)$,

(2) $\left(\mathbb{E} \otimes_{M F} \mathbb{F}\right) \otimes_{M F} \mathbb{G} \cong \mathbb{E} \otimes_{M F}\left(\mathbb{F} \otimes_{M F} \mathbb{G}\right)$ in $M F(X, \mathcal{L}, W+V+U)$, and

(3) $\mathcal{H} \mathrm{om}_{\mathrm{MF}}\left(\mathbb{E} \otimes_{M F} \mathbb{F}, \mathbb{G}\right) \cong \mathcal{H}_{\mathrm{MF}}\left(\mathbb{E}, \mathcal{H} \operatorname{Hom}_{\mathrm{MF}}(\mathbb{F}, \mathbb{G})\right)$ in $\operatorname{MF}(X, \mathcal{L}, U-V-W)$.

Proof. The isomorphisms of underlying locally free sheaves are the natural ones in each degree, taking care to observe the usual sign convention. For example, the map $\mathbb{E} \otimes_{M F} \mathbb{F} \rightarrow \mathbb{F} \otimes_{M F} \mathbb{E}$ sends a section $a \otimes b$ of $\mathcal{E}_{i} \otimes \mathcal{F}_{j}$ to $(-1)^{i j} b \otimes a$. A straightforward, but tedious, check shows that these isomorphisms commute with the differentials of the matrix factorizations.

Definition 7.5. The dual of an object $\mathbb{E}=\left(\mathcal{E}_{1} \stackrel{e_{1}}{\longrightarrow} \mathcal{E}_{0} \stackrel{e_{0}}{\longrightarrow} \mathcal{E}_{1}(1)\right)$ in $M F(X, \mathcal{L}, W)$ is the object of $M F(X, \mathcal{L},-W)$ given by

$$
\mathbb{E}^{\vee}=\mathcal{E}_{1}(1)^{\vee} \stackrel{-e_{0}^{\vee}}{\longrightarrow} \mathcal{E}_{0}^{\vee} \stackrel{e_{1}^{\vee}}{\longrightarrow} \mathcal{E}_{1}(1)^{\vee}(1),
$$

where $(-)^{\vee}$ denotes the functor $\mathcal{H}^{\vee} \mathrm{m}_{\mathcal{O}_{X}}\left(-, \mathcal{O}_{X}\right)$ and we use the canonical isomorphisms $\mathcal{E}_{1}(1)^{\vee}(1) \cong \mathcal{E}_{1}^{\vee}(-1)(1) \cong \mathcal{E}_{1}^{\vee}$.

Remark 7.6. Equivalently, $\mathbb{E}^{\vee}$ is $\mathcal{H} \operatorname{om}_{\mathrm{MF}}\left(\mathbb{E}, \mathcal{O}_{X}\right)$, where $\mathcal{O}_{X}$ denotes the matrix factorization $\left(0 \rightarrow \mathcal{O}_{X} \rightarrow 0\right)$ belonging to $M F(X, \mathcal{L}, 0)$. We also note that there is a natural isomorphism $\left(\mathbb{E}^{\vee}\right)^{\vee} \cong \mathbb{E}$.

Proposition 7.7. Let $\mathbb{E}$ and $\mathbb{F}$ be objects of $M F(X, \mathcal{L}, W)$ and $M F(X, \mathcal{L}, V)$, respectively. There is a natural isomorphism in $M F(X, \mathcal{L}, V-W)$,

$$
\mathbb{E}^{\vee} \otimes_{M F} \mathbb{F} \stackrel{\cong}{\longrightarrow} \mathcal{H o m}_{\mathrm{MF}}(\mathbb{E}, \mathbb{F}) .
$$

Proof. Recall that for locally free coherent sheaves $\mathcal{E}$ and $\mathcal{F}$, there is a canonical isomorphism $\mathcal{E}^{\vee} \otimes \mathcal{F} \cong \mathcal{H} \operatorname{om}(\mathcal{E}, \mathcal{F})$ given on sections by $\delta \otimes f \mapsto(e \mapsto \delta(e) \cdot f)$. In homological degree 1 , the isomorphism we seek is the direct sum of these canonical ones:

$$
\left[\begin{array}{cc}
\text { can } & 0 \\
0 & \text { can }
\end{array}\right]: \begin{gathered}
\mathcal{E}_{0}^{\vee} \otimes \mathcal{F}_{1} \\
\mathcal{E}_{1}^{\vee} \otimes \mathcal{F}_{0}(-1) \stackrel{\mathcal{H o m}\left(\mathcal{E}_{0}, \mathcal{F}_{1}\right)}{\longrightarrow}
\end{gathered} \begin{gathered}
\oplus \\
\mathcal{H o m}\left(\mathcal{E}_{1}, \mathcal{F}_{0}(-1)\right)
\end{gathered}
$$


In degree 0 , it is

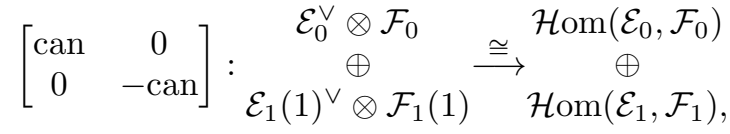

where we have also used the canonical isomorphism

$$
\mathcal{H} \operatorname{om}\left(\mathcal{E}_{1}(1), \mathcal{F}_{1}(1)\right) \cong \mathcal{H} \operatorname{om}\left(\mathcal{E}_{1}, \mathcal{F}_{1}\right) \text {. }
$$

We omit the straightforward verification that the differentials commute with these isomorphisms.

Remark 7.8. In the case $W=V$, the proposition gives an isomorphism of twisted periodic complexes.

Proposition 7.9. For matrix factorizations $\mathbb{E}$ and $\mathbb{F}$ in $M F(X, \mathcal{L}, W)$ and $M F(X, \mathcal{L}, V)$, respectively, there are natural isomorphisms

(1) $\left(\mathbb{E} \otimes_{M F} \mathbb{F}\right)^{\vee} \cong \mathbb{E}^{\vee} \otimes_{M F} \mathbb{F}^{\vee}$ in $M F(X, \mathcal{L},-W-V)$ and

(2) $\mathcal{H}_{\mathrm{om}}(\mathbb{E}, \mathbb{F})^{\vee} \cong \mathcal{H}_{\mathrm{Mm}}(\mathbb{F}, \mathbb{E})$ in $M F(X, \mathcal{L}, W-V)$.

Proof. Part (1) follows from Hom-tensor adjointness and Proposition 7.7

For (2), we have the isomorphisms

$$
\begin{gathered}
\mathcal{H} \operatorname{om}_{\mathrm{MF}}(\mathbb{E}, \mathbb{F})^{\vee} \cong\left(\mathbb{E}^{\vee} \otimes_{M F} \mathbb{F}\right)^{\vee} \cong\left(\mathbb{E}^{\vee}\right)^{\vee} \otimes_{M F} \mathbb{F}^{\vee} \\
\cong \mathbb{E} \otimes_{M F} \mathbb{F}^{\vee} \cong \mathbb{F}^{\vee} \otimes_{M F} \mathbb{E} \cong \mathcal{H} \operatorname{om}_{\mathrm{MF}}(\mathbb{F}, \mathbb{E})
\end{gathered}
$$

by Proposition 7.7 part (1) of this proposition, Remark 7.6, and Propositions 7.4(2) and 7.7, respectively.

Proposition 7.10. Let $W_{1}, \ldots, W_{4}$ be global sections of $\mathcal{L}$ and let $\mathbb{E}_{i}$ be an object of $\operatorname{MF}\left(X, \mathcal{L}, W_{i}\right)$, for $i=1, \ldots, 4$. There is an isomorphism

$$
\mathcal{H o m}{ }_{\mathrm{MF}}\left(\mathbb{E}_{1}, \mathbb{E}_{2}\right) \otimes_{M F} \mathcal{H o m}_{\mathrm{MF}}\left(\mathbb{E}_{3}, \mathbb{E}_{4}\right) \cong \mathcal{H o m}_{\mathrm{MF}}\left(\mathbb{E}_{1}, \mathbb{E}_{4}\right) \otimes_{M F} \mathcal{H o m}_{\mathrm{MF}}\left(\mathbb{E}_{2}, \mathbb{E}_{3}\right)
$$

in $M F\left(X, \mathcal{L}, W_{2}-W_{1}+W_{4}-W_{3}\right)$.

Proof. This follows immediately from Propositions 7.4 and 7.7.

Definition 7.11. The support of a complex $\mathcal{P}$ of quasi-coherent sheaves on a scheme $X$ is

$$
\operatorname{supp} \mathcal{P}=\left\{x \in X \mid \mathcal{P}_{x} \text { is not exact }\right\}=\bigcup_{i \in \mathbb{Z}} \operatorname{supp} \mathcal{H}^{i}(\mathcal{P}) .
$$

For an object $\mathcal{P}$ of $\operatorname{TPC}(X, \mathcal{L})=M F(X, \mathcal{L}, 0)$, i.e., a twisted periodic complex, we have that

$$
\operatorname{supp} \mathcal{P}=\operatorname{supp} \mathcal{H}^{0}(\mathcal{P}) \cup \operatorname{supp} \mathcal{H}^{1}(\mathcal{P})
$$

since $\mathcal{H}^{i+2} \cong \mathcal{H}^{i}(1)$. In particular, the support of a twisted periodic complex is a closed subset of $X$.

Recall that if $\mathcal{L}=\mathcal{O}_{X}$ is the trivial line bundle, then $\operatorname{TPC}\left(X, \mathcal{O}_{X}\right)$ is the category of $\mathbb{Z} / 2$-graded complexes of locally free coherent sheaves on $X$.

Lemma 7.12. Assume $X$ is a regular Noetherian separated scheme and $\mathcal{L}$ is a line bundle on $X$. For a point $x \in X$, let $k(x)$ denote its residue field and let $i_{x}: \operatorname{Spec} k(x) \rightarrow X$ be the canonical map. Given $\mathcal{P} \in T P C(X, \mathcal{L})$, a point $x \in X$ belongs to $\operatorname{supp}(\mathcal{P})$ if and only if the $\mathbb{Z} / 2$-graded complex of $k(x)$-vector spaces $i_{x}^{*}(\mathcal{P})$ is not exact. 
Proof. We can reduce immediately to the assertion that for a regular local ring $(S, \mathfrak{m}, k)$ and a (possibly unbounded) complex $\mathcal{P}$ of finitely generated free $S$-modules, $\mathcal{P}$ is exact if and only if $k \otimes_{S} \mathcal{P}$ is exact.

Let $x_{1}, \ldots, x_{n} \in S$ be a regular system of parameters. The long exact sequence in cohomology associated to the short exact sequence

$$
0 \rightarrow \mathcal{P} \stackrel{x_{n}}{\rightarrow} \mathcal{P} \rightarrow \mathcal{P} / x_{n} \mathcal{P} \rightarrow 0
$$

of complexes and Nakayama's Lemma give that $\mathcal{P}$ is exact if and only if $\mathcal{P} / x_{n} \mathcal{P}$ is exact. The result follows by induction on $n$.

Remark 7.13. The assumption that $X$ is regular is essential for Lemma 7.12 ,

Proposition 7.14. Assume $X$ is a regular Noetherian separated scheme and $\mathcal{L}$ is a line bundle on $X$. For objects $\mathcal{P}$ and $\mathcal{Q}$ of $\operatorname{TPC}(X, \mathcal{L})$, we have

$$
\operatorname{supp}\left(\mathcal{P} \otimes_{M F} \mathcal{Q}\right)=\operatorname{supp} \mathcal{P} \cap \operatorname{supp} \mathcal{Q} .
$$

Proof. For any $x \in X$ we have

$$
i_{x}^{*}\left(\mathcal{P} \otimes_{M F)} \mathcal{Q}\right) \cong i_{x}^{*}(\mathcal{P}) \otimes_{k(x)}^{\mathbb{Z} / 2} i_{x}^{*}(\mathcal{Q})
$$

where $\otimes_{k(x)}^{\mathbb{Z} / 2}$ denotes the tensor product for $\mathbb{Z} / 2$-graded complexes of $k(x)$-vector spaces (i.e., for the category $\operatorname{TPC}(\operatorname{Spec} k(x), \mathcal{O}))$. For any $\mathbb{Z} / 2$-graded complex of $k(x)$-vector spaces $V$, we have $V \cong \mathcal{H}^{0}(V) \oplus \mathcal{H}^{1}(V)[1]$. It follows that for a pair $V, W$ of such complexes, we have

$$
\begin{aligned}
V \otimes_{T P C} W \cong & \left(\mathcal{H}^{0}(V) \otimes_{k} \mathcal{H}^{0}(W)\right) \oplus\left(\mathcal{H}^{1}(V) \otimes_{k} \mathcal{H}^{1}(W)\right) \\
& \oplus\left(\mathcal{H}^{0}(V) \otimes_{k} \mathcal{H}^{1}(W)[1]\right) \oplus\left(\mathcal{H}^{1}(V) \otimes_{k} \mathcal{H}^{0}(W)[1]\right) .
\end{aligned}
$$

In particular, $V \otimes_{T P C} W$ is exact if and only if $V$ or $W$ is exact. The result follows from Lemma 7.12 .

Proposition 7.15. Assume $X$ is a regular Noetherian separated scheme and $\mathcal{L}$ is a line bundle on $X$. For an object $\mathcal{P}$ of $\operatorname{TPC}(X, \mathcal{L})$, there is an equality

$$
\operatorname{supp}(\mathcal{P})=\operatorname{supp}\left(\mathcal{P}^{*}\right)
$$

Proof. We have the isomorphism

$$
i_{x}^{*}\left(\mathcal{P}^{*}\right) \cong\left(i_{x}^{*}(\mathcal{P})\right)^{*}
$$

of $\mathbb{Z} / 2$-graded complexes of $k(x)$-vector spaces. For any such complex $V$, we have $V \cong \mathcal{H}^{0}(V) \oplus \mathcal{H}^{1}(V)[1]$ and $V^{*} \cong \mathcal{H}^{0}(V)^{*} \oplus \mathcal{H}^{1}(V)^{*}[1]$. Thus $V$ is exact if and only if $V^{*}$ is exact, using Lemma 7.12 .

\section{Stable SUPport}

In this section, returning to the context and assumptions of $\$ 2.3$, we study the properties of stable support defined in Definition 3.21. In particular, we prove Theorems 4 and 5 from the Introduction.

Recall that the stable support set of a pair $(M, N)$ of complexes of $R$-modules with bounded and finitely generated cohomology and $M$ perfect over $Q$ is defined to be

$$
\left.V_{Q}^{\mathbf{f}}(M, N)=\operatorname{supp} \operatorname{Ext}_{R} \widetilde{e v}(M, N) \cup \operatorname{supp} \operatorname{Ext}_{R} \widetilde{\text { odd }(M}, N\right) \subseteq \mathbb{P}_{R}^{\mathrm{c}-1} .
$$


If $M$ and $N$ are both perfect over $Q$, then it follows from Remark 3.5 that the stable support of $(M, N)$ may be computed as the support of a twisted-periodic complex:

$$
V_{Q}^{\mathbf{f}}(M, N)=\operatorname{supp} \mathcal{H} \operatorname{om}_{\mathrm{MF}}\left(\mathbb{E}_{M}, \mathbb{E}_{N}\right) .
$$

In the following $Q$ is assumed to be regular so that we may use Propositions 7.14 and 7.15 .

Theorem 8.1. Let $Q$ be a regular Noetherian ring of finite Krull dimension and let $R=Q /\left(f_{1}, \cdots, f_{c}\right)$ for a $Q$-regular sequence $f_{1}, \ldots, f_{c}$. For complexes of $R$ modules $M, N, M^{\prime}$, and $N^{\prime}$ with bounded finitely generated cohomology, we have

(1) $V_{Q}^{\mathbf{f}}(M, N)=\emptyset$ if and only if $\operatorname{Ext}_{R}^{n}(M, N)=0$ for all $n \gg 0$,

(2) $V_{Q}^{\mathbf{f}}(M, N) \cap V_{Q}^{\mathbf{f}}\left(M^{\prime}, N^{\prime}\right)=V_{Q}^{\mathbf{f}}\left(M, N^{\prime}\right) \cap V_{Q}^{\mathbf{f}}\left(M^{\prime}, N\right)$, and

(3) $V_{Q}^{\mathbf{f}}(M, N)=V_{Q}^{\mathbf{f}}(M, M) \cap V_{Q}^{\mathbf{f}}(N, N)=V_{Q}^{\mathbf{f}}(N, M)$.

Proof. The first assertion follows from the definition, using that for a finitely generated graded $R\left[T_{1}, \ldots, T_{c}\right]$-module $E$, the associated coherent sheaf $\widetilde{E}$ vanishes if and only if $E_{n}=0$ for all $n \gg 0$ if and only if $\operatorname{supp} \widetilde{E}=\emptyset$.

Since $Q$ is regular every $Q$-module has finite projective dimension. In particular, $V_{Q}^{\mathbf{f}}(M, N)=\operatorname{supp} \mathcal{H} \mathrm{om}_{\mathrm{MF}}\left(\mathbb{E}_{M}, \mathbb{E}_{N}\right)$ and we may use the results of the previous section. The second assertion follows from Propositions 7.10 and 7.14. Using the second assertion we have that $V_{Q}^{\mathbf{f}}(M, M) \cap V_{Q}^{\mathbf{f}}(N, N)=V_{Q}^{\mathbf{f}}(M, N) \cap V_{Q}^{\mathbf{f}}(N, M)$. By Propositions 7.9, 7.14, and 7.15 there is an equality $V_{Q}^{\mathbf{f}}(M, N)=V_{Q}^{\mathbf{f}}(N, M)$.

Although Theorem 8.1 assumes $Q$ is regular, we do not make this assumption in the rest of this section.

The singular locus of a Noetherian scheme $Z$ is

$$
\operatorname{Sing}(Z)=\left\{z \in Z \mid \mathcal{O}_{Z, x} \text { is not a regular local } \operatorname{ring}\right\} \text {. }
$$

Recall that $\gamma: Y \hookrightarrow \mathbb{P}_{Q}^{\mathrm{c}-1}$ denotes the zero subscheme of $W=\sum_{i} f_{i} T_{i} \in \mathcal{O}_{\mathbb{P}_{Q}^{\mathrm{c}-1}}(1)$. By [32, 10.2] there is a containment $\operatorname{Sing}(Y) \subseteq \mathbb{P}_{R}^{\mathrm{c}-1}$.

Lemma 8.2. For every pair $(M, N)$ of complexes of $R$-modules with bounded and finitely generated cohomology and $M$ perfect over $Q$, there is a containment

$$
V_{Q}^{\mathbf{f}}(M, N) \subseteq \operatorname{Sing}(Y) .
$$

Proof. By Corollary 3.20 and Definition 3.21, we have that

$$
V_{Q}^{\mathbf{f}}(M, N)=\operatorname{supp}{\widehat{\mathcal{E} \mathrm{xt}_{\mathcal{O}_{Y}}}}_{0}^{0}(\mathcal{M}, \mathcal{N}) \cup \operatorname{supp}{\widehat{\mathcal{E} \mathrm{xt}_{\mathcal{O}_{Y}}}}^{1}(\mathcal{M}, \mathcal{N}),
$$

where $\mathcal{M}=\beta_{*} \pi^{*} M$ and $\mathcal{N}=\beta_{*} \pi^{*} N$ in the notation of $\$ 2.3$. For any point $y \in Y$, we have

$$
\widehat{\mathcal{E x t}}_{\mathcal{O}_{Y}}^{i}(\mathcal{M}, \mathcal{N})_{y} \cong \widehat{\operatorname{Ext}}_{\mathcal{O}_{Y, y}}^{i}\left(\mathcal{M}_{y}, \mathcal{N}_{y}\right)=\operatorname{Hom}_{\mathrm{D}_{\mathrm{sg}}\left(\mathcal{O}_{Y, y}\right)}\left(\mathcal{M}_{y}, \mathcal{N}_{y}[-i]\right),
$$

where the first isomorphism is by Proposition 3.8 and the second is by Definition 2.12. If $y \notin \operatorname{Sing}(Y)$, then $\mathrm{D}_{\mathrm{sg}}\left(\mathcal{O}_{Y, y}\right)=0$, and hence ${\widehat{\mathcal{E} \mathrm{xt}_{\mathcal{O}_{Y}}^{i}}}^{i}(\mathcal{M}, \mathcal{N})_{y}=0$. 
8.1. Relation to other notions of support. In [4] Avramov and Buchweitz defined a notion of support under the additional assumption that $Q$ is local. In that case, where for simplicity we assume the residue field $k$ of $Q$ is algebraically closed, the AB-support variety of $M$ and $N$, which we write $V_{Q}^{\mathbf{f}}(M, N)^{\mathrm{AB}}$, is the union of the supports in $\mathbb{A}_{k}^{c}$ of the graded $k\left[T_{1}, \ldots, T_{c}\right]$-modules $\operatorname{Ext}_{R}^{e v}(M, N) \otimes_{R} k$ and $\operatorname{Ext}_{R}^{\text {odd }}(M, N) \otimes_{R} k$. It follows immediately from the definitions that $V_{Q}^{\mathbf{f}}(M, N){ }^{\mathrm{AB}}$ is the cone of the closed subset

$$
V_{Q}^{\mathbf{f}}(M, N) \times_{\operatorname{Spec} R} \operatorname{Spec} k \subseteq \mathbb{P}_{k}^{\mathrm{c}-1} .
$$

In particular, we deduce from Theorem 8.1 that the analogous formulas for $V_{Q}^{\mathbf{f}}(M, N)^{\mathrm{AB}}$ hold. These were first established in [4].

Benson, Iyengar, Krause defined a support for all objects in the infinite completion $\mathrm{K}(\operatorname{Inj} R)$ of $\mathrm{D}^{\mathrm{b}}(R)$ in [8]. Here $\mathrm{K}(\operatorname{Inj} R)$ is the homotopy category of injectives; see 21 for further details. For $M$ an object in $\mathrm{D}^{\mathrm{b}}(R)$, the support in the sense of [8], which we write as $V_{Q}^{\mathbf{f}}(M)^{\mathrm{BIK}}$, may be computed as

$$
V_{Q}^{\mathbf{f}}(M)^{\mathrm{BIK}}=\operatorname{supp}_{R\left[T_{1}, \ldots, T_{c}\right]} \operatorname{Ext}_{R}^{e v}(M, M) \cup \operatorname{supp}_{R\left[T_{1}, \ldots, T_{c}\right]} \operatorname{Ext}_{R}^{\text {odd }}(M, M) \subseteq \mathbb{A}_{R}^{c} .
$$

Thus $V_{Q}^{\mathbf{f}}(M, M)$ is equal to the image of $V_{Q}^{\mathbf{f}}(M)^{\mathrm{BIK}}$ under the canonical map $\mathbb{A}_{R}^{c} \backslash$ $\{0\} \rightarrow \mathbb{P}_{R}^{\mathrm{c}-1}$.

Finally, in 32, for a Noetherian separated scheme $Y$ with hypersurface singularities, Stevenson defined a support for all objects in the infinite completion $\mathrm{K}_{\mathrm{ac}}(\operatorname{Inj} Y)$ of $\mathrm{D}_{\mathrm{sg}}(Y)$; here $\mathrm{K}_{\mathrm{ac}}(\operatorname{Inj} Y)$ is the homotopy category of acyclic complexes of injective quasi-coherent sheaves on $Y$. In case $Y \hookrightarrow \mathbb{P}_{Q}^{c-1}$ is the zero subscheme of $W \in \mathcal{O}(1)$, where we assume that $Q$ is regular, then the support of a coherent sheaf $\mathcal{M}$ on $Y$, which we write as $V_{Q}^{\mathbf{f}}(\mathcal{M})^{\mathrm{St}}$, is

$$
V_{Q}^{\mathbf{f}}(\mathcal{M})^{\mathrm{St}}=\left\{x \in \operatorname{Sing} Y \mid \mathcal{M}_{x} \neq 0 \text { and } \operatorname{pd}_{\mathcal{O}_{Y, x}} \mathcal{M}_{x}=\infty\right\} \subseteq \operatorname{Sing} Y .
$$

Indeed, by [33, 8.9], support may be computed affine locally, and then the proof of [32, 5.12] shows that the support of compact objects on affine hypersurfaces takes the form above. By [32, 10.2] there is a containment Sing $Y \subseteq \mathbb{P}_{R}^{\mathrm{c}-1}$.

Proposition 8.3. Let $M$ be an object of $\mathrm{D}^{\mathrm{b}}(R)$. There is an equality

$$
V_{Q}^{\mathbf{f}}(M, M)=V_{Q}^{\mathbf{f}}(\mathcal{M})^{\mathrm{St}}
$$

where $\mathcal{M}=\beta_{*} \pi^{*} M$.

Proof. By Corollary 3.20 and Remark 3.23 we have that

$$
V_{Q}^{\mathbf{f}}(M, M)=\operatorname{supp}{\widehat{\mathcal{E} \mathrm{xt}_{\mathcal{O}_{Y}}^{0}}}^{0}(\mathcal{M}, \mathcal{M}) \text {. }
$$

The equality of support sets now follows from the isomorphism $\widehat{\mathcal{E x t}}_{\mathcal{O}_{Y}}^{0}(\mathcal{M}, \mathcal{M})_{x} \cong$ $\widehat{\operatorname{Ext}}_{\mathcal{O}_{Y, x}}^{0}\left(\mathcal{M}_{x}, \mathcal{M}_{x}\right)$, and [4, 4.2] which shows $\mathcal{M}_{x}$ has finite projective dimension if and only if $\widehat{\operatorname{Ext}}_{\mathcal{O}_{Y, x}}^{0}\left(\mathcal{M}_{x}, \mathcal{M}_{x}\right)=0$.

8.2. Realization. The following theorem answers a question posed to us by Avramov.

Theorem 8.4. Let $Q$ be a Noetherian ring of finite Krull dimension and let $R=$ $Q /\left(f_{1}, \cdots, f_{c}\right)$ for a $Q$-regular sequence $f_{1}, \ldots, f_{c}$. For every closed subset $C$ of $Y$ that is contained in $\operatorname{Sing}(Y)$ and satisfies $C \cap \operatorname{Sing}\left(\mathbb{P}_{Q}^{c-1}\right)=\emptyset$, there is an $R$-module 
$M$ such that $M$ has finite projective dimension over $Q$ and satisfies $V_{Q}^{\mathbf{f}}(M, M)=C$. In particular, if $Q$ is regular, then the result holds for every $C \subset \operatorname{Sing}(Y)$ and, in this situation, $M$ may be chosen to be an MCM R-module.

Remark 8.5. This recovers a theorem of Bergh [10] and Avramov-Iyengar [5]. When $Q$ is regular, Theorem 8.4 follows from [32, 7.11] using the relation, as sketched above, of support defined in loc. cit. to stable support.

Proof. Let $C$ be a closed subset of $\operatorname{Sing}(Y)$ such that $C \cap \operatorname{Sing}\left(\mathbb{P}_{Q}^{c-1}\right)=\emptyset$. Regard $\mathcal{O}_{C}$ as a coherent sheaf on $Y$ where $C$ is given the reduced subscheme structure. The coherent sheaf $\gamma_{*} \mathcal{O}_{C}$ is perfect on $\mathbb{P}_{Q}^{\mathrm{c}-1}$, since for any point $x$ in $\operatorname{Sing}\left(\mathbb{P}_{Q}^{\mathrm{c}-1}\right)$, we have that $\left(\gamma_{*} \mathcal{O}_{C}\right)_{x}=0$, and for any point $x$ not in $\operatorname{Sing}\left(\mathbb{P}_{Q}^{c-1}\right)$, every finitely generated $\mathcal{O}_{\mathbb{P}_{Q}^{\mathrm{c}-1}, x}$-module has finite projective dimension.

Thus $\mathcal{O}_{C}$ belongs to $\mathrm{D}_{\mathrm{sg}}^{\text {rel }}\left(Y \hookrightarrow \mathbb{P}_{Q}^{\mathrm{c}-1}\right)$ and so determines an object

$$
M \in \mathrm{D}_{\mathrm{sg}}^{\mathrm{rel}}(Q \rightarrow R)
$$

via the equivalences $\Phi: \mathrm{D}_{\mathrm{sg}}^{\mathrm{rel}}(Q \rightarrow R) \cong \mathrm{D}_{\mathrm{sg}}^{\mathrm{rel}}\left(Y \hookrightarrow \mathbb{P}_{Q}^{\mathrm{c}-1}\right)$ of Theorem 2.10, Moreover, every object of $\mathrm{D}_{\mathrm{sg}}^{\text {rel }}(Q \rightarrow R)$ is isomorphic to an $R$-module by Corollary 2.8 , and so we may assume $M$ is an $R$-module. When $Q$ is regular, every object of $\mathrm{D}_{\mathrm{sg}}^{\mathrm{rel}}(Q \rightarrow R)=\mathrm{D}_{\mathrm{sg}}(R)$ is isomorphic to an MCM $R$-module, and so we may assume $M$ is an MCM $R$-module in this case.

Let $\mathcal{M}=\beta_{*} \pi^{*} M$, in the notation of $\$ 2.3$. We have an isomorphism $\mathcal{O}_{C} \cong \mathcal{M}$ in $\mathrm{D}_{\mathrm{sg}}^{\text {rel }}\left(Y \hookrightarrow \mathbb{P}_{Q}^{\mathrm{c}-1}\right)$ since $\beta_{*} \pi^{*}$ induces an inverse equivalence of $\Phi$. Thus we have that $\mathbb{E}_{\mathcal{M}} \cong \mathbb{E}_{\mathcal{O}_{C}}$, and hence that

$$
\mathcal{H} \mathrm{om}_{\mathrm{MF}}\left(\mathbb{E}_{\mathcal{M}}, \mathbb{E}_{\mathcal{M}}\right) \cong \mathcal{H} \mathrm{om}_{\mathrm{MF}}\left(\mathbb{E}_{\mathcal{O}_{C}}, \mathbb{E}_{\mathcal{O}_{C}}\right)
$$

We wish to show that $V_{Q}^{\mathbf{f}}(M, M)=C$. By Remarks 3.23 and 3.5, and (8.6), it is enough to show that

$$
\operatorname{supp} \widehat{\mathcal{E x t}}_{\mathcal{O}_{Y}}^{0}\left(\mathcal{O}_{C}, \mathcal{O}_{C}\right)=C .
$$

The containment supp $\widehat{\mathcal{E x t}}_{\mathcal{O}_{Y}}^{0}\left(\mathcal{O}_{C}, \mathcal{O}_{C}\right) \subseteq C$ is clear. To prove the opposite containment, let $y \in C$ be the generic point of an irreducible component of the reduced subscheme associated to $C$, and observe that, since supp $\widehat{\mathcal{E x t}}_{\mathcal{O}_{Y}}^{0}\left(\mathcal{O}_{C}, \mathcal{O}_{C}\right)$ is a closed subset of $\mathbb{P}_{Q}^{\mathrm{c}-1}$, it suffices to prove $y \in \operatorname{supp} \widehat{\mathcal{E x t}}_{\mathcal{O}_{Y}}^{0}\left(\mathcal{O}_{C}, \mathcal{O}_{C}\right)$. Since $C$ is reduced and $y$ is minimal, we have $\mathcal{O}_{C, y}=A / \mathfrak{m}$, where $(A, \mathfrak{m})$ is the local ring of $Y$ at $y$. Thus

$$
\widehat{\operatorname{Ext}}_{\mathcal{O}_{Y}}^{0}\left(\mathcal{O}_{C}, \mathcal{O}_{C}\right)_{y} \cong \widehat{\operatorname{Ext}}_{A}^{0}(A / \mathfrak{m}, A / \mathfrak{m})
$$

We also have that $A=B /(f)$, for some local ring $B$ with $f$ a non-zero divisor, and $\operatorname{pd}_{B} M<\infty$. Since $y \in C \subset \operatorname{Sing}(Y), A$ is not regular, and hence $\widehat{\operatorname{Ext}}_{A}^{0}(A / \mathfrak{m}, A / \mathfrak{m}) \neq 0$.

\section{Appendix A. Orlov's Theorem}

In this appendix we establish a generalization of a theorem of Orlov [26, Theorem 2.1]. This generalization does not require the schemes in question to be defined over a ground field and drops a smoothness assumption. Our proof follows loc. cit. closely; most parts of that proof work in the more general setup.

Let $S$ be a Noetherian separated scheme of finite Krull dimension that has enough locally free sheaves (i.e., every coherent sheaf on $S$ is the quotient of a locally free 
coherent sheaf). Let $\mathcal{E}$ be a vector bundle of rank $r$ on $S$ and let $U \in \Gamma(S, \mathcal{E})$ be a regular section. Let $j: S^{\prime} \hookrightarrow S$ be the zero subscheme determined by $U$.

Let $q: X=\mathbb{P}(\mathcal{E}) \rightarrow S$ be the projective bundle corresponding to $\mathcal{E}$ and write $\mathcal{O}_{X}(1)$ for the associated line bundle on $X$. Let $W \in \Gamma\left(X, \mathcal{O}_{X}(1)\right)$ be the section induced by $U$ and let $u: Y \hookrightarrow X$ be the zero subscheme of $W$. Also consider the locally free sheaf $j^{*} \mathcal{E}$ on $S^{\prime}$, which is isomorphic to the normal bundle $\mathcal{N}_{S^{\prime} / S}$ of the inclusion of $S^{\prime}$ into $S$. Let $p: Z=\mathbb{P}\left(j^{*} \mathcal{E}\right) \rightarrow S^{\prime}$ be the corresponding projective bundle and $\mathcal{O}_{Z}(1)$ the canonical line bundle.

The canonical map $Z \rightarrow X$ factors through $Y \rightarrow X$ via a map $i: Z \rightarrow Y$. There is a short exact sequence of coherent sheaves on $Z$,

$$
0 \rightarrow \mathcal{N}_{Z / Y} \rightarrow p^{*} j^{*} \mathcal{E} \rightarrow \mathcal{O}_{Z}(1) \rightarrow 0,
$$

where $\mathcal{N}_{Z / Y}$ is the normal bundle of $Z$ in $Y$ and $p^{*} j^{*} \mathcal{E} \rightarrow \mathcal{O}_{Z}(1)$ is the canonical map.

These constructions are summarized in the following diagram:

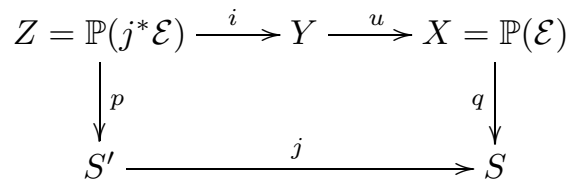

Since $p$ is flat, $\mathbf{L} p^{*}=p^{*}$ preserves boundedness of complexes. Since $i$ is a closed immersion, $\mathbf{R} i_{*}=i_{*}$ preserves coherence and boundedness. It follows that $i_{*} p^{*}$ induces a functor

$$
\Phi_{Z}=i_{*} p^{*}: \mathrm{D}^{\mathrm{b}}\left(S^{\prime}\right) \rightarrow \mathrm{D}^{\mathrm{b}}(Y) .
$$

Here, as in the body of the paper, we write $\mathrm{D}^{\mathrm{b}}(Y)$ for the bounded derived category of quasi-coherent sheaves on $Y$ with coherent cohomology.

We recall the definitions of relatively perfect complexes and the relative singularity category from Definition 2.6.

Definition A.2. Let $i: Y \hookrightarrow X$ be a closed immersion of subschemes of finite flat dimension. An object $\mathcal{F}$ in $\mathrm{D}^{\mathrm{b}}(Y)$ is a relatively perfect complex for $i$ if $i_{*} \mathcal{F}$ is a perfect complex on $X$. We write $\operatorname{RPerf}(Y \hookrightarrow X)$ for the full subcategory of $\mathrm{D}^{\mathrm{b}}(Y)$ whose objects are the relatively perfect complexes for $i$.

The category $\operatorname{RPerf}(Y \hookrightarrow X)$ is a thick subcategory of $\mathrm{D}^{\mathrm{b}}(Y)$ and there is an inclusion Perf $Y \subseteq \operatorname{RPerf}(Y \hookrightarrow X)$. Moreover, the induced functor

$$
\operatorname{RPerf}(Y \hookrightarrow X) / \operatorname{Perf} Y \rightarrow \mathrm{D}^{\mathrm{b}}(Y) / \operatorname{Perf} Y=\mathrm{D}_{\mathrm{sg}}(Y)
$$

is fully faithful. We thus identify $\operatorname{RPerf}(Y \hookrightarrow X) / \operatorname{Perf} Y$ with the corresponding full subcategory of $\mathrm{D}_{\mathrm{sg}}(Y)$.

Definition A.3. The relative singularity category of $Y$ in $X$ is

$$
\mathrm{D}_{\mathrm{sg}}^{\mathrm{rel}}(Y \subseteq X):=\operatorname{RPerf}(Y \hookrightarrow X) / \operatorname{Perf} Y .
$$

Theorem A.4. Using the notation above, the functor $\Phi_{Z}$ induces a functor

$$
\bar{\Phi}_{Z}: \mathrm{D}_{\mathrm{sg}}^{\mathrm{rel}}\left(S^{\prime} \subseteq S\right) \rightarrow \mathrm{D}_{\mathrm{sg}}^{\mathrm{rel}}(Y \subseteq X)
$$

that is an equivalence of triangulated categories. 
Remark A.5. In [26, Theorem 2.1] $S$ is assumed to be a regular scheme over a field. In this case $X$ is also regular, and hence there are equivalences $\mathrm{D}_{\mathrm{sg}}^{\mathrm{rel}}\left(S^{\prime} \subseteq S\right) \cong$ $\mathrm{D}_{\text {sg }}\left(S^{\prime}\right)$ and $\mathrm{D}_{\text {sg }}^{\text {rel }}(Y \subseteq X) \cong \mathrm{D}_{\text {sg }}(Y)$.

Proof. The functor $\Phi_{Z}$ has a right adjoint, written $\Phi_{Z}^{*}$, which is given by $\mathbf{R} p_{*} i^{\text {b }}$. Here $i^{\text {b }}$ is the right adjoint to $\mathbf{R} i_{*}$ and is given by $\mathbb{L} i_{*}(-) \otimes \omega_{Z / Y}$, where $\omega_{Z / Y}=$ $\wedge^{r-1} \mathcal{N}_{Z / Y}[-r+1]$; see [18, III.7.3]. The proof of Proposition 2.2 in [26] goes through verbatim to show that the natural map id $\rightarrow \Phi_{Z}^{*} \Phi_{Z}$ is an isomorphism and thus that $\Phi_{Z}$ is fully faithful.

Now observe that if $\mathcal{F}$ is an object of $\operatorname{RPerf}\left(S^{\prime} \hookrightarrow S\right)$, then $\Phi_{Z}(\mathcal{F})$ is an object of $\operatorname{RPerf}(Y \hookrightarrow X)$. Indeed, the diagram

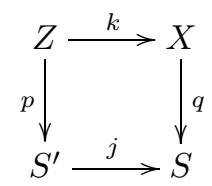

where $k=u \circ i$, is a Cartesian square and $q$ is flat. Since $j_{*} \mathcal{F}$ is perfect on $S$, we get that $q^{*} j_{*} \mathcal{F} \cong k_{*} p^{*} \mathcal{F} \cong u_{*} \Phi_{Z}$ is perfect on $X$.

Also using that $p^{*}$ and $i_{*}$ take perfect complexes to perfect complexes, we obtain an induced functor

$$
\bar{\Phi}_{Z}: \mathrm{D}_{\mathrm{sg}}^{\mathrm{rel}}\left(S^{\prime} \subseteq S\right) \rightarrow \mathrm{D}_{\mathrm{sg}}^{\mathrm{rel}}(Y \subseteq X),
$$

and this functor is fully faithful by [26, 1.1].

We now show that if $\mathcal{G}$ is in $\operatorname{RPerf}(Y \hookrightarrow X)$, then $\Phi_{Z}^{*}(\mathcal{G})$ is in $\operatorname{RPerf}\left(S^{\prime} \hookrightarrow S\right)$. We have

$$
j_{*} \Phi_{Z}^{*}(\mathcal{G})=j_{*} \mathbf{R} p_{*} i^{b} \mathcal{G} \cong \mathbf{R} q_{*} u_{*} i_{*}\left(\mathbb{L} i^{*} \mathcal{G} \otimes \omega_{Z / Y}\right) \cong \mathbf{R} q_{*} u_{*}\left(\mathcal{G} \otimes i_{*} \omega_{Z / Y}\right),
$$

where the last isomorphism uses the projection formula. Using the isomorphism $u^{*} u_{*} \mathcal{G} \cong \mathcal{G}$ and the projection formula again, we obtain

$$
u_{*}\left(\mathcal{G} \otimes i_{*} \omega_{Z / Y}\right) \cong u_{*}\left(u^{*} u_{*} \mathcal{G} \otimes i_{*} \omega_{Z / Y}\right) \cong u_{*} \mathcal{G} \otimes u_{*} i_{*} \omega_{Z / Y}
$$

Since $\omega_{Z / Y}$ is locally free on $Z$ and $i$ and $u$ have finite flat dimension, $u_{*} i_{*} \omega_{Z / Y}$ is perfect. Since we are assuming $u_{*} \mathcal{G}$ is perfect, this shows that $u_{*}\left(\mathcal{G} \otimes i_{*} \omega_{Z / Y}\right)$ is also perfect. It now follows from [1, III.4.8.1] that $j_{*} \Phi_{Z}^{*}(\mathcal{G}) \cong \mathbf{R} q_{*} u_{*}\left(\mathcal{G} \otimes i_{*} \omega_{Z / Y}\right)$ is perfect.

Since $\Phi_{Z}^{*}$ sends objects of $\operatorname{RPerf}(Y \hookrightarrow X)$ to objects of $\operatorname{RPerf}\left(S^{\prime} \hookrightarrow S\right)$, we obtain an induced functor

$$
\bar{\Phi}_{Z}^{*}: \mathrm{D}_{\mathrm{sg}}^{\mathrm{rel}}(Y \subseteq X) \rightarrow \mathrm{D}_{\mathrm{sg}}^{\mathrm{rel}}\left(S^{\prime} \subseteq S\right)
$$

that is right adjoint to $\bar{\Phi}_{Z}$.

By Lemma A.6 below, $\bar{\Phi}_{Z}^{*}$ has trivial kernel, and the rest of the proof is a formality. We have a pair of adjoint functors

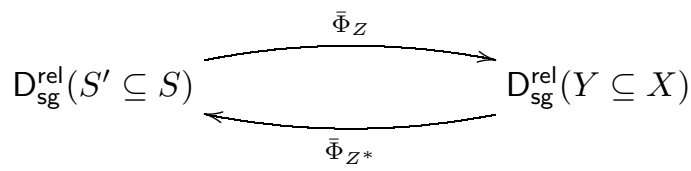

such that one is fully faithful and the kernel of the other is zero, and so the pair must be mutually inverse equivalences; see, for example the proof of Theorem 2.1 in 26. 
Lemma A.6. For $\mathcal{G} \in \mathrm{D}_{\mathrm{sg}}^{\mathrm{rel}}(Y \subseteq X)$, if $\bar{\Phi}_{Z^{*}}(\mathcal{G}) \cong 0$, then $\mathcal{G} \cong 0$.

Proof. We need to prove that if $\mathcal{G} \in \operatorname{RPerf}(Y \hookrightarrow X)$ is such that $\Phi_{Z}^{*}(\mathcal{G})$ is perfect on $S^{\prime}$, then $\mathcal{G}$ is perfect on $Y$. By [13, 6.6] we may assume that $\mathcal{G}$ is the coherent sheaf on $Y$ given by the cokernel of an object of $M F(X, \mathcal{O}(1), W)$; see Section 2 for the definition. In particular, this implies, by [13, 5.2.1], that $\mathcal{G}$ has an infinite right resolution by locally free coherent sheaves on $Y$. It follows from this that $\mathbb{L} i^{*} \mathcal{G} \cong i^{*} \mathcal{G}$ and hence $\Phi_{Z}^{*}(\mathcal{G}) \cong \mathbf{R} p_{*}\left(i^{*} \mathcal{G} \otimes \omega_{Z / Y}\right)$.

Using the short exact sequence (A.1) and [18, p. 139] we have that $\omega_{Z / Y} \cong$ $\Lambda^{r} p^{*} j^{*} \mathcal{E} \otimes \mathcal{O}_{Z}(-1)$. The projection formula gives

$$
\mathbf{R} p_{*}\left(i^{*} \mathcal{G} \otimes \omega_{Z / Y}\right) \cong \mathbf{R} p_{*}\left(i^{*} \mathcal{G}(-1)\right) \otimes \Lambda^{r} j^{*} \mathcal{E},
$$

and hence $\mathbf{R} p_{*}\left(i^{*} \mathcal{G}(-1)\right)$ is perfect on $S^{\prime}$, since $\Lambda^{r} j^{*} \mathcal{E}$ is a line bundle. By the definition of $M F(X, \mathcal{O}(1), W)$ and the equivalence $[M F(X, \mathcal{O}(1), W)] \cong$ $\mathrm{D}_{\mathrm{sg}}^{\text {rel }}\left(S^{\prime} \subseteq S\right)$ of Theorem [2.7, one sees that $\mathcal{G}(n) \cong \mathcal{G}[2 n]$ in $\mathrm{D}_{\mathrm{sg}}^{\text {rel }}\left(S^{\prime} \subseteq S\right)$ for all $n \in \mathbb{Z}$. Thus $\mathbf{R} p_{*}\left(i^{*} \mathcal{G}(n)\right)$ is perfect for all $n \in \mathbb{Z}$. It now follows from Lemma A.7 below that $i^{*} \mathcal{G}$ is perfect on $Z$.

Note that for any $x \in Y, \mathcal{G}_{x}$ has an infinite right resolution by free $\mathcal{O}_{Y, x}$-modules. Thus we may assume that we are in the following situation: let $A$ be a commutative local ring, $f_{1}, \ldots, f_{c}$ an $A$-regular sequence, set $B=A /\left(f_{1}\right), C=B /\left(f_{2}, \ldots, f_{c}\right)$, and let $M$ be a finitely generated $B$ module with an infinite free right resolution such that $M \otimes_{B} C$ has finite projective dimension over $C$. To finish the proof it is enough to show that under these conditions $M$ must be free. By [15, Lemma 0.1], the $B$-regular sequence $\left(f_{2}, \ldots, f_{c}\right)$ is also $M$-regular. Thus by [35, 4.3.12] we have $\operatorname{pd}_{B} M=\operatorname{pd}_{C}\left(M \otimes_{B} C\right)$. Finally, since $C$ has finite projective dimension over $B$, one checks that the infinite free right resolution of $M$ remains exact upon tensoring by $C$, and so $M \otimes_{B} C$ also has an infinite right free resolution. Thus $M \otimes_{B} C$ is free, and hence so is $M$ because they have the same projective dimension.

Lemma A.7 ([26, Lemma 2.6]). An object $\mathcal{G} \in \mathrm{D}^{\mathrm{b}}(Z)$ is perfect if and only if $\mathbf{R} p_{*}(\mathcal{G}(n))$ is perfect over $S^{\prime}$ for all $n \in \mathbb{Z}$.

Since we are not working over a field, the argument given in loc. cit. does not apply.

Proof. If $\mathcal{G}$ is perfect, then so is $\mathcal{G}(n)$ for all $n$, and by [1, III.4.8.1] so is $\mathbf{R} p_{*}(\mathcal{G}(n))$.

To show the converse we may work locally and assume $S^{\prime}$ is affine, so that $p$ has the form $p: Z=\mathbb{P}_{R}^{r} \rightarrow \operatorname{Spec} R$. Since $\mathcal{G}$ is perfect if and only if some syzygy in a locally free resolution of $\mathcal{G}$ is, we may assume that $\mathcal{G}$ is a coherent sheaf. Moreover, since $\mathcal{G}$ is perfect if and only if $\mathcal{G}(n)$ is perfect for some $n$, we may assume that $\mathrm{H}^{i}(Z, \mathcal{G}(n))=0$ for all $i>0$ and $n \geq 0$ by Serre's Vanishing Theorem. In particular, this implies that $\mathcal{G}$ is regular and that $\mathbf{R} p_{*} \mathcal{G} \cong p_{*} \mathcal{G}$. (See Section 5 where the definition is of regularity is recalled.)

Since $\mathcal{G}$ is regular, [30, 8.1.11] shows that there is a resolution of $\mathcal{G}$ of the form

$$
0 \rightarrow \mathcal{O}_{X}(-r+1) \otimes_{R} T_{r-1} \rightarrow \cdots \rightarrow \mathcal{O}_{X} \otimes_{R} T_{0} \rightarrow \mathcal{G} \rightarrow 0
$$

where $T_{i}$ are $R$-modules defined as follows. We set $T_{0}$ to be $p_{*} \mathcal{G}$, which is perfect by assumption, and define $Z_{1}$ to be the kernel of the map $\mathcal{O}_{X}(-1) \otimes_{R} T_{0} \rightarrow \mathcal{G}$. Note that $p_{*}\left(Z_{1}(n)\right)$ is perfect for all $n$. Inductively, set $T_{i}=p_{*}\left(Z_{i-1}(i)\right)$ and $Z_{i}$ to be the kernel of the canonical map $\mathcal{O}_{X}(-i) \otimes_{S} T_{i} \rightarrow Z_{i-1}$. By [30, p. 132] $Z_{i-1}(i)$ is regular. Using this, it follows from the definition and induction that each $T_{i}$ is 
perfect over $R$. Thus each term in the resolution (A.8) of $\mathcal{G}$ is perfect over $Z$ and so $\mathcal{G}$ must be as well.

\section{Appendix B. Stable Ext-modules and complete Resolutions}

Here we show that if $M$ is a complex with bounded finitely generated cohomology over a commutative Noetherian ring $A$, and $M$ has a complete resolution in the sense of [34, then one may compute $\widehat{\operatorname{Ext}}_{A}^{n}(M,-):=\operatorname{Hom}_{\mathrm{D}_{\mathrm{sg}}(A)}\left(\Sigma^{-n} M,-\right)$ using the complete resolution. This is well known in the case $A$ is Gorenstein by [11, and is no doubt known in the generality presented here to the experts. However, we could not find the result in the literature in the generality that we need.

Definition B.1. Let $M$ be a complex of $A$-modules with bounded and finitely generated cohomology. A complete resolution of $M$ is a diagram

$$
T \stackrel{\gamma}{\rightarrow} P \stackrel{\delta}{\rightarrow} M,
$$

where $T$ is an acyclic complex of projective $A$-modules such that $\operatorname{Hom}_{A}(T, A)$ is also acyclic, $\delta$ is a projective resolution (i.e. a quasi-isomorphism such that $P$ is a complex of projective $A$-modules with $P^{i}=0$ for $i \gg 0$ ), and $\gamma^{i}$ (the degree $i$ component of $\gamma$ ) is an isomorphism for $i \ll 0$.

Remark B.2. The previous definition was made in 34] for arbitrary complexes; see also [6].

Example B.3. Let $R=Q /\left(f_{1}, \ldots, f_{c}\right)$ be as in 2.3 , and let $M$ be a finitely generated $R$-module with finite projective dimension over $Q$, i.e. $M$ is in $\mathrm{D}_{\mathrm{sg}}^{\mathrm{rel}}(Q \rightarrow R)$. By [14, 1.2.10, 2.2.8] $M$ has finite G-dimension over $R$, and by [34, 2.4.1. 3.6] this implies that $M$ has a complete resolution in the sense above.

Lemma B.4. Let $M$ and $N$ be complexes of A-modules with bounded finitely generated cohomology and $f: M \rightarrow N$ a morphism of chain complexes such that cone $f$ is perfect. If $T \stackrel{\gamma}{\rightarrow} P \stackrel{\delta}{\rightarrow} N$ is a complete resolution of $N$, then there exists a complete resolution $T \stackrel{\gamma^{\prime}}{\rightarrow} P^{\prime} \stackrel{\delta^{\prime}}{\rightarrow} M$ of $M$ and a diagram

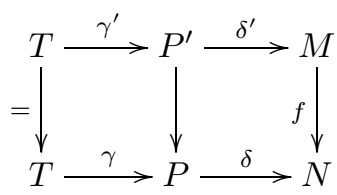

that commutes up to homotopy.

Proof. Consider the triangle

$$
M \stackrel{f}{\rightarrow} N \rightarrow \text { cone } f \rightarrow M[1]
$$

in $\mathrm{K}(A)$, the homotopy category of chain complexes of $A$-modules. Let $\delta^{\prime \prime}: F \stackrel{\simeq}{\longrightarrow}$ cone $f$ be a quasi-isomorphism from a bounded complex of finitely generated projective $A$-modules, which exists since cone $f$ was assumed perfect. Let $g: P \rightarrow F$ be a lifting over $\delta^{\prime \prime}$ of the composition $P \stackrel{\delta}{\rightarrow} N \rightarrow$ cone $f$. We can complete this to 
a map of triangles:

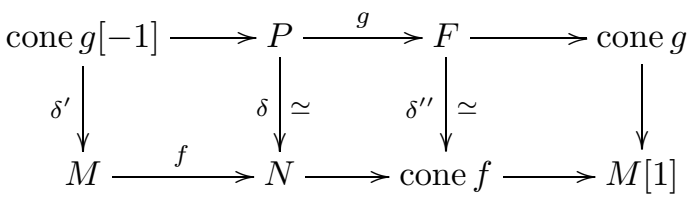

Set $P^{\prime}:=$ cone $g[-1]$. By construction $P^{\prime}$ is a complex of projective $A$-modules with $\left(P^{\prime}\right)^{i}=0$ for $i \gg 0$ and $\left(P^{\prime}\right)^{i}=P^{i}$ for $i \ll 0$. Moreover, $\delta^{\prime}$ is a quasi-isomorphism since $\delta$ and $\delta^{\prime \prime}$ are. Thus $\delta^{\prime}$ is a projective resolution of $M$.

Since $\operatorname{Hom}_{A}(T, A)$ is acyclic, and since $F$ is a bounded complex of finitely generated projective $A$-modules, it follows that $\operatorname{Hom}_{A}(T, F)$ is acyclic, i.e. there are no maps from $T$ to $F$ in $\mathrm{K}(A)$. Thus the map $\gamma: T \rightarrow P$ lifts, up to homotopy, to a map $\gamma^{\prime}: T \rightarrow P^{\prime}$. This is the complete resolution we seek.

Example B.5. Let $B$ be a local Noetherian commutative ring and $f \in B$ a nonzero divisor. Set $A=B /(f)$. By [24, Theorem 1] (see also Theorem 2.7 above) there is a fully faithful functor

$$
\text { coker : }\left[M F\left(\operatorname{Spec} B, \mathcal{O}_{B}, f\right)\right] \hookrightarrow \mathrm{D}_{\mathrm{sg}}(A) \text {. }
$$

Let $M$ be a complex of $A$-modules with bounded and finitely generated cohomology, and assume that $\mathbb{E}=\left(B^{n} \stackrel{\phi}{\rightarrow} B^{n} \stackrel{\psi}{\rightarrow} B^{n}\right)$ is a matrix factorization of $f$ such that coker $\mathbb{E} \cong M \in \mathrm{D}_{\mathrm{sg}}(A)$. Set

$$
T:=\cdots \rightarrow A^{n} \stackrel{\phi \otimes_{B} A}{\longrightarrow} A^{n} \stackrel{\psi \otimes_{B} A}{\longrightarrow} A^{n} \rightarrow \cdots .
$$

It is clear that $T \rightarrow T^{\leq 0} \rightarrow$ coker $\mathbb{E}$ is a complete resolution. By Lemma B.4 there exists some projective resolution $P \rightarrow M$ which fits into a complete resolution $T \rightarrow P \rightarrow M$.

When $A$ is Gorenstein the following is contained in [11, 6.1.2].

Lemma B.6. Let $A$ be a commutative Noetherian ring and let $M$ be an A-complex with bounded finitely generated cohomology and a complete resolution $T \stackrel{\gamma}{\rightarrow} P \rightarrow M$. Let $N$ be any A-complex with bounded finitely generated cohomology. There is an isomorphism, natural in $N$,

$$
\eta_{M}^{q}: \mathrm{H}^{q} \operatorname{Hom}_{A}(T, N) \stackrel{\cong}{\widehat{\operatorname{Ext}}_{A}^{q}}(M, N) .
$$

Moreover, there is a commutative diagram

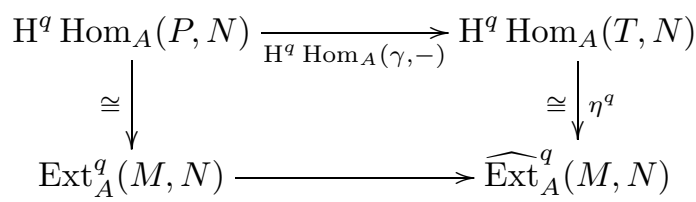

where the lower horizontal map is (2.13).

Proof. First note that we may assume that $q=0$. We view the bounded derived category of $A$-modules as the homotopy category of complexes of finitely generated projective modules with bounded cohomology. Thus we replace $M$ by $P$ and we may assume that $N$ is a complex of finitely generated projective $A$-modules. Fix an integer $k$ such that $P^{i}=0=N^{i}$ for $i \geq k$. 
There is a natural map $T \rightarrow T^{\leq k}$, and the map $\gamma: T \rightarrow P$ factors as

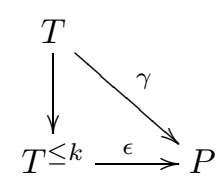

Note also that the cone of $\epsilon$ is perfect. In the remainder of this proof, we use $\simeq$ to denote a morphism whose cone is perfect. For example, we have $\epsilon: T \leq k \stackrel{\simeq}{\longrightarrow} P$.

The natural map $T \rightarrow T^{\leq k}$ induces an isomorphism

$$
\operatorname{Hom}_{A}\left(T^{\leq k}, N\right) \stackrel{\cong}{\rightarrow} \operatorname{Hom}_{A}(T, N) \text {. }
$$

Given an element $f$ in $Z^{0} \operatorname{Hom}_{A}\left(T^{\leq k}, N\right)$, we send it to the element of $\operatorname{Hom}_{\mathrm{D}_{\mathrm{sg}}(A)}(P, N)$ represented by $P \stackrel{\epsilon}{\leftarrow} T \leq k \stackrel{f}{\rightarrow} N$. If $f$ is null-homotopic, then this diagram represents the zero morphism. Thus we have a well-defined map

$$
\eta_{M}^{0}: \mathrm{H}^{0} \operatorname{Hom}_{A}(T, N) \cong \mathrm{H}^{0} \operatorname{Hom}_{A}(T \leq k, N) \rightarrow \operatorname{Hom}_{\mathrm{D}_{\mathrm{sg}}(A)}(P, N)=\widehat{\operatorname{Ext}}_{A}^{0}(M, N) .
$$

This map is independent of the choice of $k$ and thus is functorial in $N$.

We now show that the map fits into the commutative diagram above. Given an element $h \in \mathrm{H}^{0} \operatorname{Hom}_{A}(P, N) \cong \operatorname{Ext}_{A}^{0}(P, N)$, its image in $\operatorname{Hom}_{\mathrm{D}_{\mathrm{sg}}(A)}(P, N)$ is represented by $P \stackrel{=}{\longleftarrow} P \stackrel{h}{\rightarrow} N$. In the other direction, it is sent to $h \circ \gamma$ in $\mathrm{H}^{0} \operatorname{Hom}_{A}(T, N)$. By definition $\eta^{0}$ sends this to $P \stackrel{\epsilon}{\leftarrow} T^{\leq k} \stackrel{h \circ \epsilon}{\longrightarrow} N$. The diagram

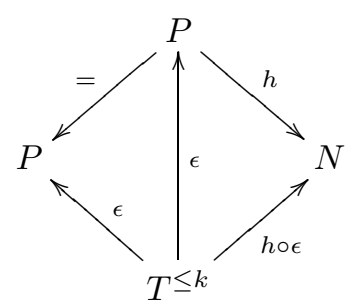

shows that the diagram commutes.

We now show that $\eta^{0}$ is an isomorphism. Suppose that $\eta(f)=0$. Then there exists a complex of projective modules $X$ and a diagram

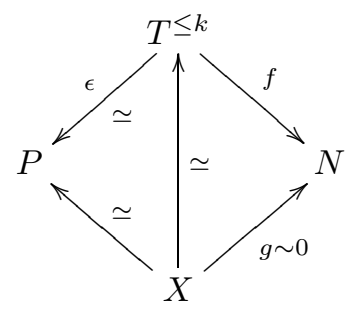

in the homotopy category. Clearly $T \rightarrow T^{\leq k}=T^{\leq k}$ is a complete resolution. By the previous lemma, there exists a complete resolution $T \rightarrow X^{\prime} \rightarrow X$ making the following diagram commute:

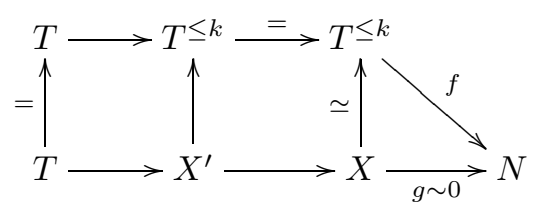


In other words we have a commutative diagram

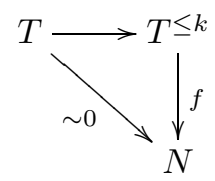

This shows that $f$ is null-homotopic, and so $\eta$ is injective.

Now let $P \stackrel{\simeq}{\longleftarrow} X \rightarrow N$ be any morphism from $P$ to $N$ in $\mathrm{D}_{\mathrm{sg}}(A)$. By the previous lemma there exists a complete resolution $T \rightarrow X^{\prime} \rightarrow X$ and a commutative diagram

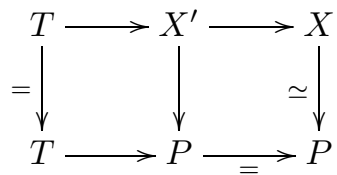

From this, one sees that there exists an integer $l$ and a commutative diagram

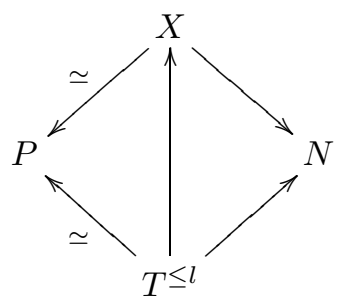

which shows that $P \stackrel{\simeq}{\longleftarrow} X \rightarrow N$ is in the image of $\eta$.

\section{ACKNOWLEDGEMENT}

It is a pleasure to thank Lucho Avramov, Ragnar-Olaf Buchweitz, David Eisenbud, Srikanth Iyengar, Daniel Murfet, and Greg Stevenson for helpful conversations about the topics in this paper.

\section{REFERENCES}

[1] Théorie des intersections et théorème de Riemann-Roch (French), Lecture Notes in Mathematics, Vol. 225, Springer-Verlag, Berlin, 1971. Séminaire de Géométrie Algébrique du BoisMarie 1966-1967 (SGA 6); Dirigé par P. Berthelot, A. Grothendieck et L. Illusie. Avec la collaboration de D. Ferrand, J. P. Jouanolou, O. Jussila, S. Kleiman, M. Raynaud et J. P. Serre. MR0354655 (50 \#7133)

[2] Luchezar L. Avramov, Obstructions to the existence of multiplicative structures on minimal free resolutions, Amer. J. Math. 103 (1981), no. 1, 1-31, DOI 10.2307/2374187. MR601460 (82m:13011)

[3] Luchezar L. Avramov and Ragnar-Olaf Buchweitz, Homological algebra modulo a regular sequence with special attention to codimension two, J. Algebra 230 (2000), no. 1, 24-67, DOI 10.1006/jabr.1999.7953. MR1774757 (2001g:13032)

[4] Luchezar L. Avramov and Ragnar-Olaf Buchweitz, Support varieties and cohomology over complete intersections, Invent. Math. 142 (2000), no. 2, 285-318, DOI 10.1007/s002220000090. MR 1794064 (2001j:13017)

[5] Luchezar L. Avramov and Srikanth B. Iyengar, Constructing modules with prescribed cohomological support, Illinois J. Math. 51 (2007), no. 1, 1-20. MR2346182 (2008j:13036)

[6] Luchezar L. Avramov and Alex Martsinkovsky, Absolute, relative, and Tate cohomology of modules of finite Gorenstein dimension, Proc. London Math. Soc. (3) 85 (2002), no. 2, 393440, DOI 10.1112/S0024611502013527. MR1912056 (2003g:16009) 
[7] Luchezar L. Avramov and Li-Chuan Sun, Cohomology operators defined by a deformation, J. Algebra 204 (1998), no. 2, 684-710, DOI 10.1006/jabr.1997.7317. MR 1624432(2000e:13021)

[8] Dave Benson, Srikanth B. Iyengar, and Henning Krause, Local cohomology and support for triangulated categories (English, with English and French summaries), Ann. Sci. Éc. Norm. Supér. (4) 41 (2008), no. 4, 573-619. MR2489634 (2009k:18012)

[9] Dave Benson, Srikanth B. Iyengar, and Henning Krause, Stratifying triangulated categories, J. Topol. 4 (2011), no. 3, 641-666, DOI 10.1112/jtopol/jtr017. MR2832572(2012m:18016)

[10] Petter Andreas Bergh, On support varieties for modules over complete intersections, Proc. Amer. Math. Soc. 135 (2007), no. 12, 3795-3803 (electronic), DOI 10.1090/S0002-9939-0709009-0. MR2341929 (2008g:13022)

[11] Ragnar-Olaf Buchweitz, Maximal Cohen-Macaulay modules and Tate-cohomology over Gorenstein rings. Unpublished manuscript, 1987.

[12] Ragnar-Olaf Buchweitz, Complete resolutions over complete intersections. Oberwolfach Rep., 8 (2011), no. 1, 543-546. Representation theory of quivers and finite dimensional algebras.

[13] Jesse Burke and Mark E. Walker, Matrix factorizations over projective schemes, Homology Homotopy Appl. 14 (2012), no. 2, 37-61. MR3007084

[14] Lars Winther Christensen, Gorenstein dimensions, Lecture Notes in Mathematics, vol. 1747, Springer-Verlag, Berlin, 2000. MR1799866 (2002e:13032)

[15] David Eisenbud, Homological algebra on a complete intersection, with an application to group representations, Trans. Amer. Math. Soc. 260 (1980), no. 1, 35-64, DOI 10.2307/1999875. MR.570778 (82d:13013)

[16] A. Grothendieck, Éléments de géométrie algébrique. III. Étude cohomologique des faisceaux cohérents. II (French), Inst. Hautes Études Sci. Publ. Math. 17 (1963), 91. MR0163911 $(29 \# 1210)$

[17] Tor H. Gulliksen, A change of ring theorem with applications to Poincaré series and intersection multiplicity, Math. Scand. 34 (1974), 167-183. MR0364232 (51 \#487)

[18] Robin Hartshorne, Residues and duality, Lecture notes of a seminar on the work of A. Grothendieck, given at Harvard $1963 / 64$. With an appendix by P. Deligne. Lecture Notes in Mathematics, No. 20, Springer-Verlag, Berlin, 1966. MR0222093 (36 \#5145)

[19] Robin Hartshorne, Algebraic geometry, Springer-Verlag, New York, 1977. Graduate Texts in Mathematics, No. 52. MR0463157(57 \#3116)

[20] M. Umut Isik, Equivalence of the derived category of a variety with a singularity category. arXiv:1011.1484.

[21] Henning Krause, The stable derived category of a Noetherian scheme, Compos. Math. 141 (2005), no. 5, 1128-1162, DOI 10.1112/S0010437X05001375. MR2157133(2006e:18019)

[22] Kevin H. Lin and Daniel Pomerleano, Global matrix factorizations, Math. Res. Lett. 20 (2013), no. 1, 91-106. MR3126725

[23] David Mumford, Lectures on curves on an algebraic surface, With a section by G. M. Bergman. Annals of Mathematics Studies, No. 59, Princeton University Press, Princeton, N.J., 1966. MR0209285 (35 \#187)

[24] Dmitri Orlov, Matrix factorizations for nonaffine LG-models, Math. Ann. 353 (2012), no. 1, 95-108, DOI 10.1007/s00208-011-0676-x. MR2910782

[25] D. O. Orlov, Triangulated categories of singularities and D-branes in Landau-Ginzburg models (Russian, with Russian summary), Tr. Mat. Inst. Steklova 246 (2004), no. Algebr. Geom. Metody, Svyazi i Prilozh., 240-262; English transl., Proc. Steklov Inst. Math. 3 (246) (2004), 227-248. MR 2101296 (2006i:81173)

[26] D. O. Orlov, Triangulated categories of singularities, and equivalences between LandauGinzburg models (Russian, with Russian summary), Mat. Sb. 197 (2006), no. 12, 117-132, DOI 10.1070/SM2006v197n12ABEH003824; English transl., Sb. Math. 197 (2006), no. 11-12, 1827-1840. MR:2437083 (2009g:14013)

[27] Dmitri Orlov, Derived categories of coherent sheaves and triangulated categories of singularities, Algebra, arithmetic, and geometry: in honor of Yu. I. Manin. Vol. II, Progr. Math., vol. 270, Birkhäuser Boston Inc., Boston, MA, 2009, pp. 503-531, DOI 10.1007/978-0-81764747-6_16. MR.2641200 (2011c:14050)

[28] Alexander Polishchuk and Arkady Vaintrob, Matrix factorizations and singularity categories for stacks, Ann. Inst. Fourier (Grenoble) 61 (2011), no. 7, 2609-2642. MR3112502

[29] Leonid Positselski, Coherent analogues of matrix factorizations and relative singularity categories. arXiv:1102.0261. 
[30] Daniel Quillen, Higher algebraic K-theory. I, Algebraic K-theory, I: Higher K-theories (Proc. Conf., Battelle Memorial Inst., Seattle, Wash., 1972), Springer, Berlin, 1973, pp. 85-147. Lecture Notes in Math., Vol. 341. MR0338129 (49 \#2895)

[31] Ian Shipman, A geometric approach to Orlov's theorem, Compos. Math. 148 (2012), no. 5, 1365-1389, DOI 10.1112/S0010437X12000255. MR2982435

[32] Greg Stevenson, Subcategories of singularity categories via tensor actions, Compos. Math. 150 (2014), no. 2, 229-272. MR3177268

[33] Greg Stevenson, Support theory via actions of tensor triangulated categories, J. Reine Angew. Math. 681 (2013), 219-254. MR3181496

[34] Oana Veliche, Gorenstein projective dimension for complexes, Trans. Amer. Math. Soc. 358 (2006), no. 3, 1257-1283 (electronic), DOI 10.1090/S0002-9947-05-03771-2. MR2187653 (2007a:16012)

[35] Charles A. Weibel, An introduction to homological algebra, Cambridge Studies in Advanced Mathematics, vol. 38, Cambridge University Press, Cambridge, 1994. MR1269324(95f:18001)

Department of Mathematics, Universität Bielefeld, 33501 Bielefeld, Germany

E-mail address: jburke@math.uni-bielefeld.de

Department of Mathematics, University of Nebraska, Lincoln, Nebraska 68588

E-mail address: mwalker5@math.unl.edu 